\title{
Production and Analysis of a Southern Ocean State Estimate
}

by

Matthew R. Mazloff

M.S. in Physics, University of Vermont, 2002

Submitted in partial fulfillment of the requirements for the degree of Master of Science in Physical Oceanography

at the

\section{MASSACHUSETTS INSTITUTE OF TECHNOLOGY \\ and the \\ WOODS HOLE OCEANOGRAPHIC INSTITUTION}

September 2006

(c) Matthew R. Mazloff, MMVI. All rights reserved.

The author hereby grants to MIT and WHOI permission to reproduce and distribute publicly paper and electronic copies of this thesis document in whole or in part.

Author ................................................. Joint Program in Physical Oceanography - Massachusetts Institute of Technology / Woods Hole Oceanographic Institution August 11, 2006 Certified by .

Carl Wunsch

Cecil and Ida Green Professor of Physical Oceanography Thesis Supervisor Accepted by 


\title{
Production and Analysis of a Southern Ocean State Estimate
}

\author{
by \\ Matthew R. Mazloff \\ Submitted to the Joint Program in Physical Oceanography - Massachusetts \\ Institute of Technology / Woods Hole Oceanographic Institution \\ on August 11, 2006, in partial fulfillment of the \\ requirements for the degree of \\ Master of Science in Physical Oceanography
}

\begin{abstract}
A modern general circulation model of the Southern Ocean with one-sixth of a degree resolution is optimized to the observed ocean in a weighted least squares sense. Convergence toward the state estimate solution is carried out by systematically adjusting the control variables (prescribed atmospheric state, initial conditions, and open northern boundary at $24.7^{\circ} \mathrm{S}$ ) using the adjoint method. A cost function compares the model state to data from CTD synoptic sections, hydrographic climatology, satellite altimetry, and XBTs. Costs attributed to control variable perturbations ensure a physically realistic solution. An optimized solution is determined by the weights placed on the cost function terms. The state estimation procedure, along with the weights used, is described. A significant result is that the adjoint method is shown to work at eddy-permitting resolution in the highly-energetic Southern Ocean. At the time of the writing of this thesis the state estimate was not fully consistent with the observations. An analysis of the remaining misfit, as well as the mass transport in the preliminary state, is presented.
\end{abstract}

Thesis Supervisor: Carl Wunsch

Title: Cecil and Ida Green Professor of Physical Oceanography 


\section{Acknowledgments}

I am grateful to Carl Wunsch, who continually shows students what a young science physical oceanography is. Carl has inspired me to study the oceans using the tool of state estimation. He has provided guidance, resources, and support. I am especially grateful to Carl for allowing me to be part of the ECCO consortium.

I am grateful to Patrick Heimbach for spending countless hours teaching me the ECCO model. Without Patrick's immense support none of this work would be possible. Working with Carl, Patrick, and the rest of the MIT ECCO project has been more than a great learning experience; it has been fun. I have enjoyed the work that has resulted in this thesis; for this I am most appreciative.

I am grateful for all the people who make the MIT-WHOI Joint Program tic. Being a student in this program is a great experience. The administrators, fellow students, and professors all deserve recognition in this work, either for intellectual or recreational reasons (everyone needs a break!). At the risk of being neglectful, I will give a short list of people who are deserving of special thanks. Thanks to the best

office mates in the world: Bea, Gerbi, Stephanie, and Yowchi. These friends, along with Alex, Ariane, Sutherland, Tatiana, and the man@mit have kept me sane (or at least made me feel normal for being insane). Jake Gebbie, Joe Pedlosky, and John Toole have been excellent educational resources. Thanks to the intramural sports captains, especially Alaskapeter. And thanks to the 1417 Aesthetics Committee. I'm sure I have overlooked people worthy of this list; I hope that my actions have made these people aware of my appreciation.

Finally I thank Cori and my wonderful family who are always supportive of me. 


\section{Contents}

1 Introduction $\quad 9$

1.1 Synopsis of Thesis . . . . . . . . . . . . . . . . . . . 12

1.2 The Southern Ocean: An Introduction . . . . . . . . . . . . . . 13

2 A Model-Observation Least-Squares Optimization 17

2.1 Introduction . . . . . . . . . . . . . . . . . 17

2.2 The Cost Function . . . . . . . . . . . . . . . . . . . . . . . . . . 18

2.3 Method of Lagrange Multipliers . . . . . . . . . . . . . . . . . . . . . 19

2.4 The Normal Equations . . . . . . . . . . . . . . . . . . . . 22

2.5 Solution Method for the Normal Equations . . . . . . . . . . . . . . 23

3 Model Specifics $\quad 25$

3.1 The Forward Model . . . . . . . . . . . . . . . . . . . . 25

3.2 Forward Model Inputs . . . . . . . . . . . . . . . . . . . 27

3.2 .1 Topography . . . . . . . . . . . . . . . 27

3.2 .2 Atmospheric State . . . . . . . . . . . . . . . . 30

3.2.3 Initial and Northern Boundary Conditions . . . . . . . . . . . 31

3.3 The Adjoint Model . . . . . . . . . . . . . . . . . . . . . . 32

3.4 Gradient Descent . . . . . . . . . . . . . . . . 34

4 Observations and Uncertainties 35

4.1 Observations . . . . . . . . . . . . . . . . . . . 35

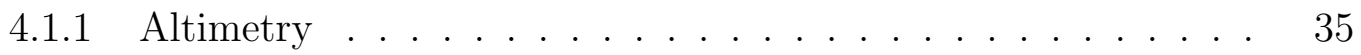


4.1 .2 Climatology . . . . . . . . . . . . . . . . . . 36

4.1 .3 CTD and XBT In Situ Data . . . . . . . . . . . . . . 36

4.1.4 Sea Surface Temperature . . . . . . . . . . . . . . . . 36

4.2 Uncertainty . . . . . . . . . . . . . . . . . . . 37

4.3 Estimated Uncertainty in the Observations . . . . . . . . . . . 38

4.3 .1 Altimetry . . . . . . . . . . . . . . . . . . . 38

$4.3 .2 \quad$ In Situ Data . . . . . . . . . . . . . . . . . . . . 40

4.3 .3 Climatology . . . . . . . . . . . . . . . . . . . . 41

4.3.4 Sea Surface Temperature . . . . . . . . . . . . . . . . . 42

4.4 Estimated Uncertainty in the Controls . . . . . . . . . . . . . 42

4.4.1 Prescribed Atmospheric State Uncertainty . . . . . . . . . . 43

4.4 .2 Initial Condition Uncertainty . . . . . . . . . . . . . . 44

4.4.3 Open Northern Boundary Condition Uncertainty . . . . . . 44

5 Production of a Southern Ocean State Estimate 47

5.1 Feasibility of Eddy-Permitting Southern Ocean State Estimation . . . 48

5.2 Model-Observation Misfit. . . . . . . . . . . . . . . . . . 51

5.2 .1 Climatology . . . . . . . . . . . . . . . . 53

5.2 .2 Altimetry . . . . . . . . . . . . . . . . . . 57

$5.3 \quad$ Summary $\ldots \ldots \ldots \ldots \ldots \ldots \ldots \ldots \ldots \ldots$

6 The Southern Ocean General Circulation $\quad 61$

6.1 Introduction . . . . . . . . . . . . . . . . . . . . 61

6.2 Zonal Transport . . . . . . . . . . . . . . . . . 63

6.3 Meridional Transports . . . . . . . . . . . . . . . 67

6.4 Discussion . . . . . . . . . . . . . . . . . . 82

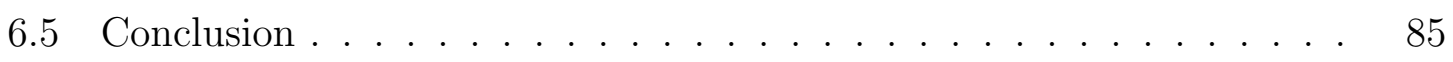

$\begin{array}{lr}\text { A Formulation of the Residual Mean Circulation } & 89\end{array}$ 


\section{Chapter 1}

\section{Introduction}

Representing roughly a third of the world's oceans, the mid- and high-latitude Southern Hemisphere oceans play a significant role in determining the global climate. These waters are responsible for linking the Atlantic, Indian, and Pacific Oceans. They are the world's largest oceanic sink of $\mathrm{CO}_{2}$ (Patra et al.; 2005) and wind energy (Wunsch; 1998). The signature of water masses formed in this ocean are found throughout the world(Marsh et al.; 2000). Despite the Southern Ocean's significance, the circulation and variability of these waters are poorly understood.

One reason for this lack of understanding is a deficiency of observations in this remote area. Fortunately, the validity of this justification has been eroding over the last half century. Observations of the Southern Ocean ${ }^{1}$ were rare prior to 1962. Observations became more frequent after 1962, yet they were often focused on specific regions, for example the Drake Passage. Largely due to the introduction of satellite altimeters, observational coverage has dramatically increased in both space and time over the last fifteen years. The data influx was significantly increased again in 2002 with the introduction of hundreds of autonomous floats through the Argo program (Gould and the Argo Steering Team; 2004).

Even with available observations, the task of describing the Southern Ocean cir-

\footnotetext{
${ }^{1}$ For purposes of the Antarctic Treaty, the International Hydrographic Organization has defined the Southern Ocean to extend from Antarctica north to $60^{\circ} \mathrm{S}$. The region of study in this thesis is the oceans south of latitude $25^{\circ} \mathrm{S}$, which, for ease, will be referred to collectively as the Southern Ocean.
} 
culation is not trivial. The Southern Ocean is extremely energetic. In the Southern Ocean, more than in any other ocean, eddies play a significant role in the dynamics giving observations large transient signals that must be diagnosed. Eddy dynamics occur on the order of the Rossby deformation radius, which, for the Southern Ocean, is on the order of $18 \mathrm{~km}$ (Marshall et al.; 1993). Modeling the Southern Ocean is made difficult by these facts; Southern Ocean models must either have high-resolution or rely heavily on eddy parameterizations.

Large data sets of Southern Ocean observations are available. Descriptions of the Southern Ocean flow coming from inferences from these in situ (e.g. Marsh et al. (2000)) and satellite (e.g. Gille $(1995,1997)$ ) observations give a basic understanding of the density structure, mass transport, and variability in the Southern Ocean. Most in situ observations were taken primarily in select regions. Though this hides the general Southern Ocean circulation, it does allow bounds to be placed on the transport across sections (e.g. the Drake Passage (Cunningham et al.; 2003)).

Models have also provided information about the processes occurring in the Southern Ocean circulation (e.g. The FRAM Group (1991), Lee and Coward (2003), and Hallberg and Gnanadesikan (2005)). Combining information from both models and observations has resulted in the development of a well established picture of the Southern Ocean circulation. This picture has been divided into one circulation in the horizontal plane (Figure 1 of Olbers et al. (2004)), and one circulation in the meridional-vertical plane (Figure 12 of Olbers et al. (2004)). The horizontal picture shows two circumpolar currents, one flowing along the Polar Front and one along the Subantarctic Front. Poleward of these currents one finds the Ross and Weddell Gyres. Subtropical gyres are found equatorward of the circumpolar currents. Interactions between the circumpolar currents and the subtropical gyres is implied, the most notable of which is the outflow of circumpolar water at the eastern boundaries. The picture in the meridional-vertical slice shows water entering the Southern Ocean and gradually upwelling. This inflow is returned in either a surface or abyssal outflow. The vertical extent of these outflows is significantly smaller than the inflow, suggesting outflow velocity is rather large. 
The simplification of the Southern Ocean circulation that these two pictures imply are a strong statement about the current understanding of the Southern Ocean circulation. The simplification, however, is useful as it aids the development of insightful idealized theories (e.g. Marshall and Radko (2005) and Olbers and Visbeck (2005)). These theories often attempt to bridge the gap between models and observations. Analysis of model output, however, shows large discrepancies from the established picture (Marsh et al.; 2000). This raises questions as to whether it is even possible to simplify the entire Southern Ocean general circulation into two-dimensional pictures. Model analysis also raises questions as to whether model results are consistent with each other (Russell et al.; 2005), and, more importantly, if they are consistent with the observations.

The Fine Resolution Antarctic Model (FRAM) was very influential in guiding the perception and understanding of the Southern Ocean. This model was developed in attempt to elucidate the Southern Ocean circulation and transport. Determining water mass formation and the basic dynamic balance of the Antarctic Circumpolar Current were also stated goals (Stevens and Stevens; 1999). The FRAM project resulted in numerous papers, which both increased comprehension of the Southern Ocean, and further developed model analysis tools. A complete quantitative comparison of FRAM to observations of the Southern Ocean was never carried out. The accuracy of the FRAM results, and thus many of the inferences drawn from the analysis of these results is unknown (Saunders and Thompson; 1993). It is not worthwhile to list the shortcomings of FRAM; the FRAM project was very successful. It is necessary, however, to move forward with the investigation of the Southern Ocean. This new investigation should use, as its primary tool, a modern ocean model that has been rigorously constrained to observations.

The intention of this work is to bring a state-of-the-art eddy-permitting Southern Ocean model into consistency with observations. Analysis of this state estimate can be used both to describe the Southern Ocean's general circulation, and also to bring the study of its dynamics to a higher level. The state estimate will provide for the unprecedented ability to test current inferences and theories regarding the Southern 
Ocean.

\subsection{Synopsis of Thesis}

As Southern Ocean observations become more abundant, the ability to synthesize these disparate data types into models is a primary concern. Model-observation synthesis provides a reference for observational data analysis. For example, a state estimate solution can provide a proper reference frame to deduce mixing from dye release programs. Determining the state of the ocean also allows one to observe climate trends, which in a region like the Southern Ocean, may have significant global implications. Furthermore, the tool of state estimation aids the physical oceanographer in understanding the mixing, evolution, and transport pathways of various water masses. A knowledge of these processes in the Southern Ocean is crucial to diagnosing the meridional overturning of freshwater, heat, and mass in the world's oceans (Sloyan and Rintoul; 2001b).

A global state estimate has been developed by the consortium to Estimate the Circulation and Climate of the Ocean (ECCO) (Stammer et al.; 2002). This state estimate, however, is not eddy-permitting; its resolution may be too coarse to shed light on the dominant dynamics occurring in the highly energetic Southern Ocean. It was not obvious, however, that a Southern Ocean model of adequate resolution to permit eddies could be brought into consistency with observations. A significant result of this thesis is that the model solution has been brought considerably closer to consistency with observations by formulating the problem into a least-squares optimization with Lagrange multipliers. This well documented model-data synthesis procedure is widely referred to in this oceanographic context as the adjoint method(Wunsch; 1996; Wunsch and Heimbach; 2005b). Chapter 2 describes the adjoint method. Specifics of the individual components involved in this method, which include the forward model, the adjoint model, and the gradient decent optimization routine, are described in Chapter 3. Chapter 4 describes the observations used. Model uncertainty (described in the context of controls) and observational uncertainty are also discussed in Chapter 
4.

This thesis describes the design and production of a model solution to the Southern Ocean (see Section 3.1). This model is initially run with what is arguably the most realistic boundary and initial conditions available. The initial solution, however, was found to be not fully consistent with observations. The solution has now been brought closer to observations by the optimization procedure. Chapter 5 describes the degree of consistency of the current state estimate, shedding light on where the estimate is acceptable and where improvement is needed. To some extent, this chapter chronicles the experience of producing a high-resolution Southern Ocean state estimate. Chapter 6 describes the general circulation of the Southern Ocean as deduced from analysis of the existing state estimate. The zonal transport across three meridional sections is evaluated. A zonally integrated meridional overturning streamfunction is constructed and analyzed. The water mass transport fluxed into and out of the Southern Ocean is quantified and compared to previous estimates. The reader is likely to conclude that the analysis carried out in Chapter 6 raises more questions than it answers. It is the hope of the author that the converged Southern Ocean state estimate will be used as a tool to address these questions in future work.

The remainder of this chapter presents the basic physical characteristics of the Southern Ocean. The purpose is to familiarize the reader with the terminology used in the state estimate analysis, and also to draw awareness to the importance of studying this Ocean.

\subsection{The Southern Ocean: An Introduction}

The circulation of the Southern Ocean is driven primarily by the wind. It is estimated that greater than $70 \%$ of the work done by the wind on the world's oceans occurs on the $30 \%$ of its surface found polewards of $40^{\circ} \mathrm{S}$ (Wunsch; 1998). The majority of this wind work results from the large zonal wind-stress component. How the Southern Ocean reacts to this large zonal momentum flux is a matter of debate (see for example (Johnson and Bryden; 1989; Warren et al.; 1998; Olbers et al.; 2004)). 
Substantial wind forcing combined with the lack of a complete meridional boundary results in the Southern Ocean's prominent circumpolar mass transport. This transport, which is one of the largest of the all the world's oceans, is known as the Antarctic Circumpolar Current. This system of currents moves waters at speeds on the order of $0.1 \mathrm{~m} / \mathrm{s}$, and reaches to depths deeper than $2 \mathrm{~km}$ (Tomczak and Godfrey; 1994). Significant bottom velocities in the ACC cause it to be greatly affected by topography (Tansley and Marshall; 2001). Ridge structures, such as the Scotia Ridge found to the east of the Drake Passage, alter the large-scale flow of the ACC and cause a local increase in small-scale turbulence (Garabato et al.; 2004).

The wind forcing acts to separate the Southern Ocean into several regions. While large isopycnal tilts are the principal indicator of fronts, Orsi et al. (1995) gave property (temperature, salinity, oxygen) indicators to determine the Southern Ocean frontal locations. Using these indicators, they were then able to give a description of the meridional extent and structure of the fronts that divide these regions from compiled historical data. A brief description of these regions follows ${ }^{2}$. Closest to the Antarctic Shelf, the Subpolar Zone (also sometimes called the Antarctic Zone) is characterized by westward winds ${ }^{3}$ and a corresponding a poleward Ekman transport. Several anti-cyclonic gyres, the Ross Gyre and the Weddell Gyre being the most notable, are found in this weakly stratified zone. Strong eastward winds are found north of the Subpolar Zone. These winds, which span the latitudes between approximately $65^{\circ} \mathrm{S}$ and $35^{\circ} \mathrm{S}$, drive an equatorward Ekman transport on the order of 30 Sverdrups $\left(1 \mathrm{~Sv}=10^{6} \mathrm{~m}^{3} / \mathrm{s}\right)$. This region of equatorward Ekman transport is split into a Polar Front Zone and a Subantarctic Front Zone. The Polar front marks the divergence region separating the Polar Front Zone and the Subpolar Zone. The Subantarctic Front separates the Polar Front Zone and the Subantarctic Front Zone. Much of the Antarctic Circumpolar Current transport occurs along the Polar and Subantarctic Fronts. To the north of the Subantarctic Front Zone there is a region

\footnotetext{
${ }^{2}$ It should be emphasized that the description given was derived by compiling historical data into a time mean climatology. The coverage of this data varies widely in space and time; it is possible that a state estimate may reveal a very different picture.

${ }^{3}$ Wind directions given are temporal means; snapshots of wind patterns over the Southern Ocean are very complex
} 
of westward winds driving waters poleward. The Subtropical Front, which is a region of convergence, marks the northern extent of the Subantarctic Front Zone. There are significant water property changes across the Subtropical Front because the subtropical gyre waters found to the north are much warmer and saltier than waters in the Subantarctic Front Zone. Frontal locations are influenced by many factors, determining what sets their exact position, a question first examined by Deacon in 1937, remains unresolved (Cunningham et al.; 2003).

One is unable to explain the features of the Southern Ocean using wind forcing alone; the Southern Ocean dynamics are effected by surface fluxes of both mass and buoyancy. Because evaporation is small in the Southern Ocean, mass input is dominated by a relatively high precipitation band centered around $50^{\circ} \mathrm{S}$. Poleward of this band. buoyancy forcing is dominated by ice dynamics; wintertime ice formation results in brine rejection, while summertime ice melt results in freshwater input (Tomczak and Godfrey; 1994). The buoyancy and winds combine to facilitate a great deal of water mass conversion and formation. The waters formed ventilate a substantial fraction of the world's oceans, making the Southern Ocean a key component in the global overturning circulation (Rintoul et al.; 2001).

A simplified picture of the water masses, taken primarily from the studies of Orsi et al. (1995), Sloyan and Rintoul (2001a), and Tomczak and Liefrink (2005), is given in the following paragraph. There are discrepancies between the studies largely resulting from the variability in space and time of the data used. Finding consistency in the disparate data sets, as is done with a state estimate, may allow a unified picture of Southern Ocean water masses to emerge.

The surface waters of the Subantarctic Front Zone are known as Subantarctic Mode Waters (SAMW). Because these waters are formed during deep late winter convection they are characterized by low potential vorticity and high oxygen. Cold and fresh SAMW ventilates the thermocline of the subtropical gyres by subducting at the Subtropical Front. Nutrient rich Upper Circumpolar Deep Water (UCDW) flows into the Southern Ocean at depth and is upwelled in the vicinity of the Polar Front. Buoyancy forcing and mixing with SAMW converts much of this water to Antarctic 
Intermediate Water (AAIW). This conversion is thought to occur primarily in the Southeast Pacific and southwest Atlantic regions. AAIW, which is characterized by a mid-depth salinity minimum, subducts along the Subantarctic Front such that it lies beneath the SAMW in the Subantarctic Front Zone. Lower Circumpolar Deep Water (LCDW) flows into the Southern Ocean at depths below the fresher UCDW. (North Atlantic Deep Water is associated with Circumpolar Deep Water.) Much of the LCDW upwells to become the surface waters of the Subpolar Zone. At several locations around Antarctica, particularly in the Ross and Weddell Seas, this water mass mixes with near freezing shelf waters and newly convected waters to form Antarctic Bottom Water. Antarctic Bottom Water, which is the densest water mass in the world oceans, gains heat and salinity through mixing as it moves out across the Polar and Subantarctic Front Zone. The resulting mixture makes up a large percentage of bottom water in the world's oceans.(Orsi et al.; 1995; Sloyan and Rintoul; 2001a; Tomczak and Liefrink; 2005) 


\section{Chapter 2}

\section{A Model-Observation \\ Least-Squares Optimization}

\section{$2.1 \quad$ Introduction}

With the goal of Estimating the Circulation and Climate of the Ocean, the ECCO consortium was founded in 1998 as part of the World Ocean Circulation Experiment (WOCE) (Stammer et al.; 1999). Their goal is to combine a modern ocean circulation model with diverse ocean observations in order to produce quantitative accounts of the global ocean state. In contrast to so-called numerical weather prediction, these estimates will include the ocean's history as well as predictions. ECCO's efforts toward this goal include a "production run" and several other projects. The existing ECCO-GODAE production run is a $1^{\circ}$ resolution, 23 depth level, global ocean state estimate running from 1992 through 2004. Wunsch and Heimbach (2005a,b) describe the preliminary results and explore their scientific impacts.

Model-data misfit in the ECCO-GODAE production run is greatest in the highly energetic regions, likely due to lack of resolution. Eddy parameterization schemes currently used in low resolution models inadequately reproduce high resolution model results (Hallberg and Gnanadesikan; 2005). A recent ECCO project proved the ability to carry out a high resolution state estimate with open ocean boundaries using the adjoint method (Gebbie; 2004; Gebbie et al.; 2005). The state estimate being 
carried out for this thesis builds on other ECCO projects. This project is in league with ECCO's goal of carrying out accurate high-resolution state estimates over large regions in order to better understand the oceans (Menemenlis et al.; 2003).

The forward model used, as described in Section 3.1, represents an approximation to ocean physics. It's assumed the model dynamics have a relatively small uncertainty with respect to the true ocean dynamics. There is, however, a significant level of uncertainty in the model inputs. Perturbations to these input fields control the model solution, and are thus referred to as the controls. The control vectors, which are initially zero, are iteratively solved for in the optimization process. The resulting model trajectory, which is within the combined model and data uncertainty, is the state estimate. (The optimization being carried out does not address the significant level of uncertainty in the parameterizations of small scale ocean processes. These parameterizations (see Section 3.1) also control the model solution.)

The performance of the state estimate is reduced to one number through the cost function. This function is a sum of squared model-data misfit terms and squared control vector magnitude terms. Every term has an appropriate weighting attributed to it. The task at hand is to minimize this weighted sum of squares. ${ }^{1}$

In the following section, the cost function is described. Section 2.3 describes the adjoint method, which is used to minimize the cost function. The weighting of the cost function terms, which is largely responsible for the state estimate solution, is described in Chapter 4. Notation used in this thesis is adopted primarily from Wunsch (1996). For brevity and simplicity, the time step, $\Delta \mathrm{t}$, is set to 1 in all equations.

\subsection{The Cost Function}

The cost function used to gauge the quality of our state estimate is written out in its entirety on page 20. Boldface denotes a matrix or vector field. Overbars denote an averaging, and primes denote a deviation from an average. The weighting matrices

\footnotetext{
${ }^{1}$ Reduction of the cost to an acceptable value is not the only requirement for a successful state estimate; see Section5.2
} 
are all denoted as W's for data-model misfit and as Q's for control penalty terms. The first three terms, (2.1a-2.1b), are model state misfits from in situ data. The following term, (2.1c), is the model sea surface temperature (SST) misfit from observations. The model sea surface height (SSH) anomaly and SSH mean misfits from altimetric observations are given as terms (2.1d-2.1e). The next two terms, (2.1f), are the mean model state misfit to hydrographic climatology. The following twelve terms, $(2.1 \mathrm{~g}$ 2.1q), are penalties applied to the magnitude of the control vectors. The last two terms, (2.1r), are a smoothness constraint placed on the control vector. Chapter 4 further elaborates on this cost function by describing the controls, observations, and weights used.

\subsection{Method of Lagrange Multipliers}

The ECCO consortium has examined several methods for carrying out state estimates. Their experience suggests the method of Lagrange multipliers, widely known in the oceanographic community as the adjoint method, is practical for this thesis. This method has proven success at eddy-permitting resolution (Gebbie et al.; 2005). The ECCO group has spent considerable time ensuring both the methods computational efficiency and robustness on a variety of supercomputer platforms (Heimbach et al.; 2005). Furthermore, the availability of semi-automatic differentiation tools allows the model setup time will not be excessive (Giering and Kaminski; 1998).

The adjoint method is, formally, a weighted least squares with Lagrange multiplier optimization problem. The goal is to minimize the cost function, which may be written as

$$
\begin{gathered}
\tilde{J}=\sum_{t=0}^{t_{f}}[\mathbf{E}(t) \mathbf{x}(t)-\mathbf{y}(t)]^{T} \mathbf{W}(t)[\mathbf{E}(t) \mathbf{x}(t)-\mathbf{y}(t)] \\
+\sum_{t=0}^{t_{f-1}} \mathbf{u}(t)^{T} \mathbf{Q}(t) \mathbf{u}(t)
\end{gathered}
$$

where the first summation represents all the model-data misfit terms, (2.1a-2.1f), and the second summation represents the control penalty terms, $(2.1 \mathrm{~g}-2.1 \mathrm{r}) . \mathbf{x}(t)$ is the state vector of temperature, salinity, velocity, and sea surface height. $\mathbf{y}(t)$ is 


$$
\begin{aligned}
& \tilde{J}=\sum_{t}^{12}\left(\overline{\mathbf{T}}-\overline{\mathbf{T}}_{c t d}\right)^{T} \mathbf{W}_{T_{i n s}}\left(\overline{\mathbf{T}}-\overline{\mathbf{T}}_{c t d}\right)+\left(\overline{\mathbf{S}}-\overline{\mathbf{S}}_{c t d}\right)^{T} \mathbf{W}_{S_{i n s}}\left(\overline{\mathbf{S}}-\overline{\mathbf{S}}_{c t d}\right) \\
& +\sum_{t}^{12}\left(\overline{\mathbf{T}}-\overline{\mathbf{T}}_{x b t}\right)^{T} \mathbf{W}_{T_{i n s}}\left(\overline{\mathbf{T}}-\overline{\mathbf{T}}_{x b t}\right) \\
& +\sum_{t}^{12}\left(\overline{\mathbf{T}}_{s f c}-\overline{\mathbf{T}}_{R e y}\right)^{T} \mathbf{W}_{S S T}\left(\overline{\mathbf{T}}_{s f c}-\overline{\mathbf{T}}_{R e y}\right) \\
& +\sum_{t}^{366}\left(\eta^{\prime}-\eta_{\mathbf{t p}}^{\prime}\right)^{T} \mathbf{W}_{\mathbf{t p}}\left(\eta^{\prime}-\eta_{\mathbf{t p}}^{\prime}\right)+\left(\eta^{\prime}-\eta_{\mathbf{e r s}}^{\prime}\right)^{T} \mathbf{W}_{\mathbf{t p}}\left(\eta^{\prime}-\eta_{\mathbf{e r s}}^{\prime}\right) \\
& +\left(\bar{\eta}-\bar{\eta}_{\text {grace }}\right)^{T} \mathbf{W}_{\text {geoid }}\left(\bar{\eta}-\bar{\eta}_{\text {grace }}\right) \\
& +\sum_{t}^{12}\left(\overline{\mathbf{T}}-\overline{\mathbf{T}}_{c l m}\right)^{T} \mathbf{W}_{T_{c l m}}\left(\overline{\mathbf{T}}-\overline{\mathbf{T}}_{c l m}\right)+\left(\overline{\mathbf{S}}-\overline{\mathbf{S}}_{c l m}\right)^{T} \mathbf{W}_{S_{c l m}}\left(\overline{\mathbf{S}}-\overline{\mathbf{S}}_{c l m}\right) \\
& +\left(\mathbf{T}_{i n i}-\mathbf{T}_{0}\right)^{T} \mathbf{Q}_{T_{0}}\left(\mathbf{T}_{i n i}-\mathbf{T}_{0}\right)+\left(\mathbf{S}_{i n i}-\mathbf{S}_{0}\right)^{T} \mathbf{Q}_{S_{0}}\left(\mathbf{S}_{i n i}-\mathbf{S}_{0}\right) \\
& +\sum_{t}^{37}\left(\mathbf{T}_{o . b .}-\mathbf{T}_{\text {o.b.ECCO}}\right)^{T} \mathbf{Q}_{T_{\text {o.b. }}}\left(\mathbf{T}_{o . b .}-\mathbf{T}_{\text {o.b.ECCO}}\right) \\
& +\sum_{t}^{37}\left(\mathbf{S}_{o . b .}-\mathbf{S}_{o . b . E C C O}\right)^{T} \mathbf{Q}_{S_{o . b .}}\left(\mathbf{S}_{o . b .}-\mathbf{S}_{o . b . E C C O}\right) \\
& +\sum_{t}^{37}\left(\mathbf{U}_{o . b .}-\mathbf{U}_{o . b \cdot E C C O}\right)^{T} \mathbf{Q}_{V_{o . b .}}\left(\mathbf{U}_{o . b .}-\mathbf{U}_{o . b \cdot E C C O}\right) \\
& +\sum_{t}^{37}\left(\mathbf{V}_{o . b .}-\mathbf{V}_{\text {o.b.ECCO}}\right)^{T} \mathbf{Q}_{V_{\text {o.b. }}}\left(\mathbf{V}_{\text {o.b. }}-\mathbf{V}_{\text {o.b.ECCO}}\right) \\
& +\sum_{t}^{1464}\left(\mathbf{U w i n d}-\mathbf{U w i n d}_{n c e p}\right)^{T} \mathbf{Q}_{U w i n d}\left(\mathbf{U w i n d}-\mathbf{U w i n d}_{n c e p}\right) \\
& +\sum_{t}^{1464}\left(\mathbf{V w i n d}-\mathbf{V w i n d}_{n c e p}\right)^{T} \mathbf{Q}_{V w i n d}\left(\mathbf{V w i n d}-\mathbf{V w i n d}_{n c e p}\right) \\
& +\sum_{t}^{1464}\left(\mathbf{T a t m}-\mathbf{T a t m}_{n c e p}\right)^{T} \mathbf{Q}_{\text {Tatm }}\left(\mathbf{T a t m}-\mathbf{T a t m}_{n c e p}\right) \\
& +\sum_{t}^{1464}\left(\mathbf{s p f h}-\mathbf{s p f h}_{n c e p}\right)^{T} \mathbf{Q}_{s p f h}\left(\mathbf{s p f h}-\mathbf{s p f h}_{n c e p}\right) \\
& +\sum_{t}^{1464}\left(\text { rain }-\operatorname{rain}_{n c e p}\right)^{T} \mathbf{Q}_{\text {rain }}\left(\text { rain }-\operatorname{rain}_{n c e p}\right) \\
& +\sum_{t}^{1464}\left(\mathbf{s w r}-\mathbf{s w r} \mathbf{r}_{n c e p}\right)^{T} \mathbf{Q}_{s w r}\left(\mathbf{s w r}-\mathbf{s w r} \mathbf{r}_{n c e p}\right) \\
& +\sum_{t}^{37}\left(\nabla^{\mathbf{2}} \mathbf{u}_{\mathbf{a f}}\right)^{T} \mathbf{Q}_{a f s m t h}\left(\nabla^{\mathbf{2}} \mathbf{u}_{\mathbf{a f}}\right)+\left(\nabla^{\mathbf{2}} \mathbf{u}_{\mathbf{o}}\right)^{T} \mathbf{Q}_{\text {ini smth }}\left(\nabla^{\mathbf{2}} \mathbf{u}_{\mathbf{o}}\right)
\end{aligned}
$$


the set of observations and $\mathbf{E}(t) \mathbf{x}(t)$ is the model estimate of the observations. $\mathbf{u}(t)$ is the control vector of perturbations to the boundary conditions, initial conditions, and atmospheric state. Theoretically, $\mathbf{u}(t)$ should contain all model variables subject to adjustment, including compensation for model error. In practice, model error is compensated by increasing the tolerance for observational error. This compensation does not change the problem mathematically, but it is important to differentiate these sources of error when interpreting results. $\mathbf{W}(t)$ and $\mathbf{Q}(t)$ are weighting matrices.

The state solved for, $\mathbf{x}(t)$, must be a solution of the forward model. Thus the problem at hand is to minimize (2.2) subject to the constraint:

$$
\mathbf{x}(t+1)=\mathcal{L}[\mathbf{x}(t), \mathbf{B q}(t), \mathbf{\Gamma} \mathbf{u}(t)]
$$

Here the operator $\mathcal{L}$ represents the full non-linear forward model (over 80,000 lines of fortran code). $\mathbf{q}(t)$ are the first guess boundary conditions and forcing, and again, $\mathbf{u}(t)$ are the perturbations to this vector. $\mathbf{B}$ and $\boldsymbol{\Gamma}$ map the effects of these vectors into the model.

Cost function extrema occur when $\partial \tilde{J} / \partial \mathbf{x}(t)$ and $\partial \tilde{J} / \partial \mathbf{u}(t)$ vanish. If there are $\mathrm{M}$ state variables and $\mathrm{N}$ controls, $\mathrm{M}+\mathrm{N}$ equations must be satisfied. The state, however, is determined by the controls, and thus there are only $\mathrm{N}$ degrees of freedom. The system is overdetermined; there is likely to be no solution. Yet the cost function does have an absolute minimum; the goal of the method of Lagrange multipliers is to find this state.

To find the constrained minimum of the cost function a vector of Lagrange multipliers, $\mu$, is introduce and used to append the model constraint to the cost function:

$$
\begin{aligned}
J= & \sum_{t=0}^{t_{f}}[\mathbf{E}(t) \mathbf{x}(t)-\mathbf{y}(t)]^{T} \mathbf{W}(t)[\mathbf{E}(t) \mathbf{x}(t)-\mathbf{y}(t)] \\
& +\sum_{t=0}^{t_{f-1}} \mathbf{u}(t)^{T} \mathbf{Q}(t) \mathbf{u}(t) \\
& -2 \sum_{t=0}^{t_{f-1}} \mu(t+1)^{T}\{\mathbf{x}(t+1)-\mathcal{L}[\mathbf{x}(t), \mathbf{B q}(t), \Gamma \mathbf{u}(t)]\}
\end{aligned}
$$

The model constraint always holds, ensuring that the appended cost function has the same value as the original function regardless of the value of $\mu$. A new vector, 
$\mu$, of $\mathrm{M}$ independent unknown elements, and thus $\mathrm{M}$ new degrees of freedom, have now been introduced. The problem now has $\mathrm{M}+\mathrm{N}$ degrees of freedom, and is no longer overdetermined. The M Lagrange multipliers take the value that ensures the vanishing of the $\mathrm{M}$ equations: $\partial J / \partial \mathbf{x}(t)=0$. The problem has been reduced to an unconstrained optimization problem where $\mathbf{u}$ and $\mu$ can be solved for to minimize $J$. This is a standard excercise; the procedure is to set the derivatives of (2.4) equal to zero and solve the resulting normal equations.

\subsection{The Normal Equations}

Taking the derivative of (2.4) with respect to $\mathbf{x}, \mathbf{u}$, and $\mu$ yields the normal equations:

$$
\frac{1}{2} \frac{\partial J}{\partial \mu(t)}=0 \Rightarrow \quad \mathbf{x}(t+1)=\mathcal{L}[\mathbf{x}(t), \mathbf{B q}(t), \boldsymbol{\Gamma u}(t)], \quad 0 \leq t \leq t_{f}-1
$$

$$
\begin{aligned}
\frac{1}{2} \frac{\partial J}{\partial \mathbf{x}(t)}=0 \Rightarrow \quad \mu(t)= & (\partial \mathcal{L} / \partial \mathbf{x}(t))^{T} \mu(t+1) & 0 \leq t \leq t_{f} \\
& +\mathbf{E}(t)^{T} \mathbf{W}(t)[\mathbf{E}(t) \mathbf{x}(t)-\mathbf{y}(t)], & \mu(0)=\mathbf{0}=\mu\left(t_{f}+1\right)
\end{aligned}
$$

$$
\frac{1}{2} \frac{\partial J}{\partial \mathbf{u}(t)}=0 \Rightarrow \quad \mathbf{u}(t)=-\mathbf{Q}(t)(\partial \mathcal{L} / \partial(\mathbf{u}))^{T} \boldsymbol{\Gamma}^{T} \mu(t+1), \quad 0 \leq t \leq t_{f}-1
$$

Equation (2.5) is the non-linear forward ocean model to be described in Section 3.1. The second and third equation combine to yield the so-called adjoint model. (The task of running the adjoint model is elaborated on in Section 3.3). The Lagrange multipliers, $\mu$, are the independent variables solved for by the adjoint model in accordance with equation (2.6). The dynamical operator acting on $\mu$ is $(\partial \mathcal{L} / \partial \mathbf{x}(t))^{T}$. The adjoint model is forced by the model-observation misfit, $\mathbf{E}(t) \mathbf{x}(t)-\mathbf{y}(t)$. The adjoint model uses the third equation to relate the Lagrange multipliers to the cost function gradient with respect to the controls. This can be more easily seen by rewriting equation 
$(2.7)$ as

$$
\frac{1}{2} \frac{\partial J}{\partial \mathbf{u}(t)}=\mathbf{Q}(t)^{-1} \mathbf{u}(t)+(\partial \mathcal{L} / \partial(\mathbf{u}))^{T} \boldsymbol{\Gamma}^{T} \mu(t+1)
$$

In total there are $\mathrm{M}+\mathrm{M}+\mathrm{N}$ equations $((2.5)+(2.6)+(2.7))$ and $\mathrm{M}+\mathrm{M}+\mathrm{N}$ variables $(\mu+\mathbf{x}+\mathbf{u})$. The procedure of solving this formally just-posed problem is described in the following section.

Before moving on, it is insightful to further analyze equation (2.8) in the following way. Consider a control vector, $\mathbf{u}^{*}(t)$, that is an additive perturbation to the model state. The cost function, equation (2.4), would become

$$
\begin{aligned}
& J=\quad \sum_{t=0}^{t_{f}}[\mathbf{E}(t) \mathbf{x}(t)-\mathbf{y}(t)]^{T} \mathbf{W}(t)[\mathbf{E}(t) \mathbf{x}(t)-\mathbf{y}(t)] \\
& +\sum_{t=0}^{t_{f-1}} \mathbf{u}(t)^{T} \mathbf{Q}(t) \mathbf{u}(t) \\
& -2 \sum_{t=0}^{t_{f-1}} \mu(t+1)^{T}\left\{\mathbf{x}(t+1)-\mathcal{L}[\mathbf{x}(t), \mathbf{B q}(t), \boldsymbol{\Gamma} \mathbf{u}(t)]-\mathbf{u}^{*}(t)\right\}
\end{aligned}
$$

The normal equation for this new variable would be

$$
\frac{1}{2} \frac{\partial J}{\partial \mathbf{u}^{*}(t)}=\mu(t+1)
$$

This example helps elucidate the physical meaning of the Lagrange multipliers. The Lagrange multipliers reveal the sensitivity of the cost function to perturbations in the controls. Were $\mathcal{L}$ linear in control space, each Lagrange multiplier would yield the influence of each control on the cost function directly. Though non-linearities of the model dynamics makes the relationship between cost function gradients and Lagrange multipliers more complicated, Equation (2.8) shows that the Lagrange multipliers are able to give the needed information on the direction and relative amplitude the controls should be perturbed in order to optimize the cost.

\subsection{Solution Method for the Normal Equations}

Equations (2.5-2.7) are non-linear, and thus not directly solvable using standard algorithms (e.g. Gaussian elimination). Trumping the non-linearity issue, however, is the problem of dimension as the control space being optimized has over 200 million 
degrees of freedom. The solution is to solve the equations iteratively.

The procedure is as follows: the ocean circulation model is run forward and the ocean state is calculated. This step has ensured equation (2.5) is satisfied. During this step, the cost function, equation (2.4) is calculated making $\mathbf{E}(t)^{T} \mathbf{W}(t)[\mathbf{E}(t) \mathbf{x}(t)-\mathbf{y}(t)]$ known. Considering equation (2.6), it is now possible to determine $\mu$ at all times by integrating backward from $t_{f}$. This integration is known as the adjoint model. As for initial conditions, $\mu\left(t_{f}+1\right)=0$ as there is no cost function sensitivity to the controls at time $t_{f}+1$, indeed there are no cost function terms at this time. With $\mu$ known, Equation (2.8) can be used to solve for $\partial J / \partial \mathbf{u}(t)$. Using these gradients, a quasiNewtonian optimization routine (Gilbert and Lemaréchal; 1989) solves for appropriate perturbations to the hundreds of millions of control terms in order to navigate the modeled state towards a cost function minimum (see Section 3.4). Iterative runs allow convergence to model input parameters that give a physically realistic state of the ocean most consistent with the observed ocean.

There is still one issue: the transposed partial derivatives of the forward model with respect to the control and state variables, $(\partial \mathcal{L} / \partial(\mathbf{u}))^{T}$ and $(\partial \mathcal{L} / \partial \mathbf{x}(t))^{T}$, must be determined. This is not a trivial exercise. The efficient calculation of the adjoint code has been made feasible thanks to the existence of automatic differentiation (AD) tools. Giering and Kaminski (1998) have provided an AD tool, known as Transformation of Algorithms in Fortran (TAF), which takes the gradients of the forward model with respect to the control and state variables by rigorously applying the chain rule, line by line, to the forward code. The resulting code allows calculation of the gradients of the cost function with respect to the controls. TAF does have pitfalls, but the forward ocean model has been developed to be compatible with it (Marotzke et al.; 1999). Certain coding structures are avoided, and the result, after much work, is the ability to automatically generate the adjoint model code (Heimbach et al.; 2005). 


\section{Chapter 3}

\section{Model Specifics}

\subsection{The Forward Model}

The dynamics of the world's oceans are accurately described by the Navier-Stokes equations. Some terms in the full set of these governing equations have been shown to have a small contribution to the ocean's dynamical balances. These insignificant terms are neglected in order to efficiently model the equations, and to more easily interpret the results. The simplifications used in this study include the Boussinesq approximation, which assumes density perturbations are negligible unless multiplied by gravity. This approximation reduces conservation of mass to conservation of volume. The momentum equations are simplified by neglecting the Coriolis acceleration terms that are thought to be small. In the vertical momentum equation, other terms are neglected by making the hydrostatic approximation. These assumptions lead to a set of equations known as the hydrostatic primitive equations (HPEs). The HPEs are appropriate for this study as they have been found to give essentially the same numerical model solution as the full set of incompressible Navier-Stokes equations at $1^{0}$ horizontal resolution(Marshall, Hill, Perelman and Adcroft; 1997). It is arguable that the refined model resolution in this study necessitates the addition of some of the omitted Coriolis and metric terms. It is assumed, however, that the effect these terms would have on the solution is negligible.

The MIT Ocean General Circulation Model (MITgcm) (Marshall, Adcroft, Hill, 
Perelman and Heisey; 1997; Marshall, Hill, Perelman and Adcroft; 1997; Adcroft et al.; 2006) is used to solve the HPEs on a "C" grid (Arakawa and Lamb; 1977). Computational resources are limited, and thus so too is model resolution. The model is unable to reproduce ocean dynamics occurring on scales on the order of the gridspacing, and the effect of these small-scale processes on resolved dynamics must be parameterized. The parameterizations implemented in the MITgcm, and used in this study include the Gent-McWilliams Redi eddy parameterization, and the non-local K-profile (KPP) vertical mixing parameterization. (Adcroft et al.; 2006)

The Gent-McWiliams Redi parameterization considers the effect of geostrophic eddies as two separate processes, and thus involves two separate parameterizations. The first parameterization represents the mixing of tracers along isentropes and takes the form of a diffusive operator acting parallel to density surfaces (Redi). The second parameterization (Gent-McWiliams) represents an adiabatic advection by eddies. The advective velocity used in this parameterization is a function of the slope of the density surfaces. For the Southern Ocean state estimate the along isopycnal diffusive and advective flux coefficients are both set to $2 \mathrm{~m}^{2} \mathrm{~s}^{-1}$.

The KPP scheme is implemented to account for vertical mixing caused by both wind stirring and convection (Large et al.; 1994). In this scheme the vertical fluxes are parameterized as $\overline{w^{\prime} x^{\prime}}=-\kappa_{x}\left(\partial \bar{x} / \partial z-\gamma_{x}\right)$ where $x$ is some tracer quantity (temperature or salinity in this state estimate). The turbulent diffusivity, $\kappa_{x}$, is given as a function of the turbulent velocity scale, $w^{*}$, and a non-dimensional shape function, $\mathcal{G}(\sigma)$, such that $\kappa_{x}=h w^{*}(\sigma) \mathcal{G}\{\sigma)$. Here $\sigma=z / h$ where $h$ is the depth of the turbulent boundary layer determined as the location where the bulk Richardson number exceeds some critical value. $\gamma_{x}$ is the non-local flux term implemented to represent the fact that scalar fields may be largely homogeneous in convective boundary layers, yet fluxes may still be finite. The MITgcm modeling community has had success representing proper physics with this parameterization (Gebbie; 2004).

For this thesis, the model is setup in spherical coordinates with $1 / 6^{\circ}$ horizontal resolution and 42 vertical levels of varying depth (see Section 3.2.1). A 1,200 second time-step is used. Laplacian viscosity and diffusivities are used with $\nu_{h}=10^{3}$ and 
$\kappa_{h}=10^{3}$ in the horizontal, and with $\nu_{v}=10^{-3}$ and $\kappa_{v}=10^{-3}$ in the vertical. A biharmonic horizontal viscosity of $10^{9}$ is also implemented. No-slip boundary conditions are used. Compared to other forward models of this resolution, the set-up uses high diffusivities and viscosities (Saunders and Thompson; 1993). This was done initially to make sure the adjoint method would be successful and is discussed further in Section 3.3.

\subsection{Forward Model Inputs}

\subsubsection{Topography}

The Southern Ocean bathymetry file was derived by first binning and averaging, on a $1 / 4^{\circ}$ grid, the high-resolution topography data (GTOPO30) of Smith and Sandwell (Smith and Sandwell; 1997). This data covers the domain equatorward of $\sim 72^{\circ} S$. Topography for the remainder of the domain comes from the ETOP05 data set (NOAA; 1988.). This $1 / 4^{\circ}$ field is then interpolated to fit the $1 / 6^{\circ}$ model grid. In this sense, the bathymetry used is slightly smoothed. Some degree of bathymetric smoothing is desirable in the absence of a correct bottom boundary layer parameterization (Penduff et al.; 2002).

The model is discretized on a finite volume grid; the bathymetry can only be fit as well as the model grid allows. The model uses 42 depth levels, and employs partial cells such that the cell may be cropped to fit the bottom bathymetry. Table 3.1 shows the minimum and maximum possible thickness for each level. The depth profile, exactly as represented by the model, is shown in Figure 3-1. The maximum depth thickness for each level was chosen carefully with three considerations in mind. The first being that the cell thickness difference between neighboring layers would be no larger than 10\%. (Large telescoping differences in model grids can cause unphysical wave refractions.) The second consideration was regarding the vertical gradients of the observed ocean temperature and salinity (T-S) properties. The upper ocean has larger T-S gradients, and thus a finer resolution is desired for the upper $\sim 1,000$ 
meters. The final consideration is of the topographic structure in the region of study. Ideally, one would well resolve the upper ocean, slowly telescoping out at depth. One would again increase the resolution upon approaching the bottom boundary layer. The location of the bottom boundary changes depending on location, however, and thus one cannot resolve this region efficiently in a model that uses a fixed vertical coordinate. Analysis of the Southern Ocean bathymetry reveals the ocean depth to be greater than 2,500 meters over much of the region. For this reason, the greatest cell thickness occurs around 2,000 meters.

Model bathymetry can greatly affect the flow field (Losch and Wunsch; 2003). The bathymetry used in many modeling efforts, including the ECCO-GODAE production run, has been tuned to ensure model transports are realistic in well observed ocean constriction points, for example in the Drake Passage and Florida Strait (Lu et al.; 2002). It is noteworthy that no tuning of the topography, or cropping of the ocean shelfs, has been carried out in this present state estimate. 


\begin{tabular}{|c|c|c|c|}
\hline z level & Min. $\Delta z[\mathrm{~m}]$ & $\operatorname{Max} . \Delta \mathrm{z}[\mathrm{m}]$ & $\begin{array}{l}\text { Depth of middle of cell }[\mathrm{m}] \\
\quad(\text { assumes max. } \Delta \mathrm{z})\end{array}$ \\
\hline 1 & 10 & 10 & -5.0 \\
\hline 2 & 11 & 11 & -15.5 \\
\hline 3 & 12 & 12 & -27.0 \\
\hline 4 & 13 & 13 & -39.5 \\
\hline 5 & 14 & 14 & -53.0 \\
\hline 6 & 16 & 16 & -68.0 \\
\hline 7 & 18 & 18 & -85.0 \\
\hline 8 & 20 & 20 & -104.0 \\
\hline 9 & 23 & 23 & -125.5 \\
\hline 10 & 26 & 26 & -150.0 \\
\hline 11 & 29 & 29 & -177.5 \\
\hline 12 & 33 & 33 & -208.5 \\
\hline 13 & 37 & 37 & -243.5 \\
\hline 14 & 42 & 42 & -283.0 \\
\hline 15 & 48 & 48 & -328.0 \\
\hline 16 & 50 & 55 & -379.5 \\
\hline 17 & 50 & 63 & -438.5 \\
\hline 18 & 50 & 72 & -506.0 \\
\hline 19 & 50 & 82 & -583.0 \\
\hline 20 & 50 & 94 & -671.0 \\
\hline 21 & 50 & 108 & -772.0 \\
\hline 22 & 50 & 124 & -888.0 \\
\hline 23 & 50 & 142 & -1021.0 \\
\hline 24 & 50 & 163 & -1173.5 \\
\hline 25 & 56.1 & 187 & -1348.5 \\
\hline 26 & 64.5 & 215 & -1549.5 \\
\hline 27 & 74.1 & 247 & -1780.5 \\
\hline 28 & 85.2 & 284 & -2046.0 \\
\hline 29 & 78.6 & 262 & -2319.0 \\
\hline 30 & 75.0 & 250 & -2575.0 \\
\hline 31 & 75.0 & 250 & -2825.0 \\
\hline 32 & 75.0 & 250 & -3075.0 \\
\hline 33 & 75.0 & 250 & -3325.0 \\
\hline 34 & 75.0 & 250 & -3575.0 \\
\hline 35 & 75.0 & 250 & -3825.0 \\
\hline 36 & 75.0 & 250 & -4075.0 \\
\hline 37 & 75.0 & 250 & -4325.0 \\
\hline 38 & 75.0 & 250 & -4575.0 \\
\hline 39 & 75.0 & 250 & -4825.0 \\
\hline 40 & 75.0 & 250 & -5075.0 \\
\hline 41 & 75.0 & 250 & -5325.0 \\
\hline 42 & 75.0 & 250 & -5575.0 \\
\hline
\end{tabular}

Table 3.1: Vertical grid. The maximum depth is 5,700 meters. 


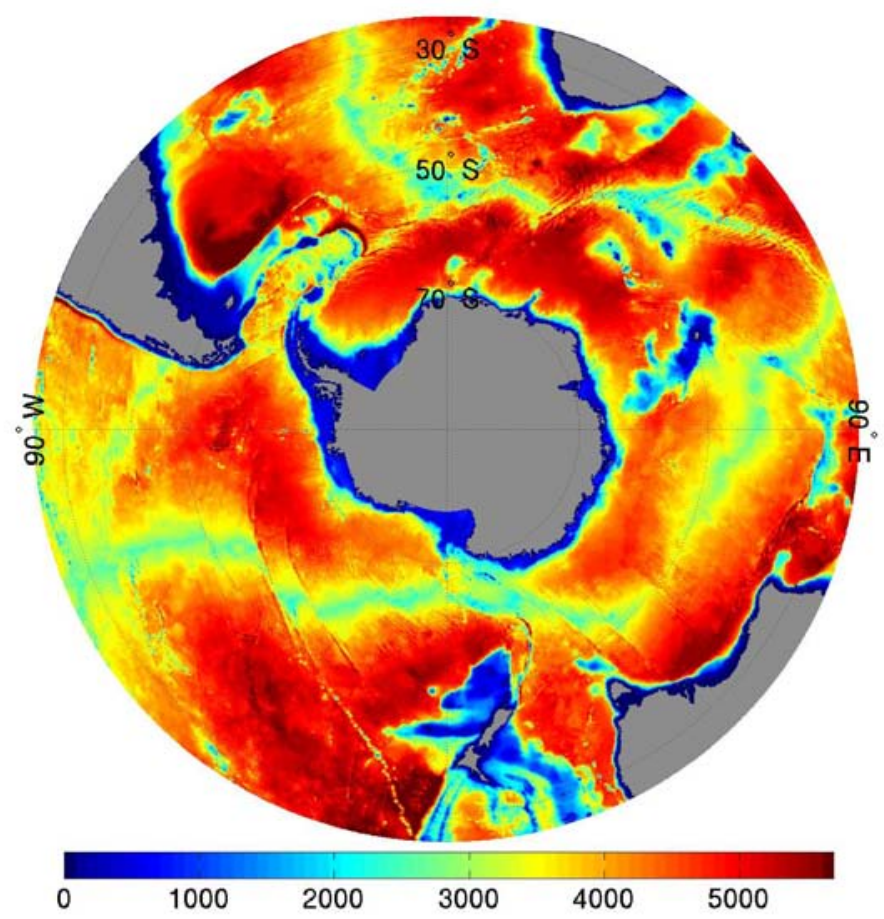

Figure 3-1: Model bathymetry [meters]

\subsubsection{Atmospheric State}

The initial estimate of the atmospheric state is obtained from a re-analysis of historical data produced in a joint effort between the National Centers for Environmental Prediction and the National Center for Atmospheric Research (the NCEP/NCAR Reanalysis Project, (Kalnay and coauthors; 1996)). The following fields, which are available at 6 hourly intervals, are used to prescribe the atmospheric state:

- Air temperature [Kelvin] at 2 meters

- Specific humidity [kilogram water vapor / kilogram air] at 2 meters

- Zonal wind speed [meters / second] at 10 meters

- Meridional wind speed [meters / second] at 10 meters

- Precipitation [meters / second]

- Short wave radiative flux [watts / meter $^{2}$ ]

- Long wave radiative flux [watts $/$ meter $^{2}$ ] 
A monthly river (freshwater) runoff climatology is also prescribed in the model. This climatology was determined by analyzing both observed river runoff and the climatological difference between evaporation and precipitation over continents (Fekete et al.; 2002). The prescribed atmospheric state is compared to the model ocean state using bulk formulas (Large and Pond; 1982) to determine appropriate momentum, heat, and mass fluxes (see Adcroft et al. (2006) for more information).

\subsubsection{Initial and Northern Boundary Conditions}

The initial conditions are derived from the ECCO-GODAE production run (Iteration 163). The mean January 1999 ECCO-GODAE state was interpolated to the model grid. Using the appropriate prescribed atmospheric state and northern boundary conditions, the model is run forward for a year. The first month of the spin-up shows a significant geostrophic adjustment, as well as a large increase in the zonal transport. This zonal transport levels off in September and then begins to decrease throughout the remainder of the year. The reason for the decrease in transport appears to be the establishment of strong currents near Antarctica (opposing the ACC transport) during the latter half of the year. The meridional transport shows a large jump in January, but is relatively constant for the remaining eleven months of the year. The model state is stored on January 1, 2000 to be used as the first guess initial conditions.

The northern extent of this regional state estimate, located at $24.7^{\circ} \mathrm{S}$, has sections of open ocean. The ocean state at this location must be prescribed. The dynamical balances near land boundaries are different than those found in the ocean interior. Boundary regions are characterized by strong velocity gradients as the flow is brought to rest. These gradients often lead to significantly stronger turbulent dissipation and vertical motions than are found in the open ocean. The prescribed northern boundary condition is not one of vanishing velocity. Boundary effects may still occur, however, if the prescribed conditions are inconsistent with the interior circulation. An improper prescribed northern boundary condition therefore leads to unphysical

boundary features. Previous regional models have included so-called sponge layers at open boundaries to damp these unphysical features. Sponge layers are unphysical 
themselves, however, and they are absent in this state estimate. As the model is constraint to the observed ocean, it is the hope that the adjoint method will determine a northern boundary condition consistent with the interior.

A first guess northern boundary condition was taken directly from the ECCOGODAE production run (Iteration 163). The production run monthly mean state (velocity, temperature, and salinity) was interpolated onto the model grid, and then linearly interpolated in time such that a northern boundary condition is prescribed at every model time-step. This first guess northern boundary conditions should be reasonably consistent with the initial run as the first guess initial conditions are derived from the same state estimate.

\subsection{The Adjoint Model}

As explained in Chapter 2, the calculation of the adjoint model code from the MITgcm code is possible using the AD tool TAF. The code TAF produces is the exact adjoint of the forward model code with two exceptions. The adjoint of the Gent-McWilliams Redi parameterization and the KPP mixed layer parameterization are omitted. For the Gent-McWilliams Redi parameterization this omission is rather insignificant, as the effect of this scheme has been reduced substantially by choosing a background along isopycnal mixing coefficient of $2 \mathrm{~m}^{2} \mathrm{~s}^{-1}$. A standard value of $\sim 1,000 \mathrm{~m}^{2} \mathrm{~s}^{-1}$ is used in coarse resolution models (Visbeck et al.; 1997; Ferreira et al.; 2005), however this value is reduced as model resolution approaches the deformation radius. There is a good deal of uncertainty in the choice of the mixing coefficient; the appropriate way to deal with this parameterization would be to have the optimization determine the coefficient.

Availability of the adjoint model code does not guarantee success of the optimization. Cost function complexity increases with both model run duration and model non-linearity. A complex cost function makes finding its absolute minimum difficult. Several strategies have been employed to ensure the success of this state estimate. Previous experience shows the number of iterations required to converge to an accept- 
able cost function minimum may be significantly reduced by employing a multi-scale approach, where one uses information from a state estimate of lower resolution or shorter temporal duration for a new state estimate. Using this knowledge, the initial and northern boundary conditions for this estimate were derived from the the ECCO-GODAE $1^{\circ}$ optimization (as explained in the previous section). Analogous to this multi-scale approach, this project begins by using a relatively viscous model to reduce model non-linearity. The hope is that a multi-viscosity approach will be analogous to the proven multi-scale approach. The idea is to lower the viscosity in the model when the high-viscosity model state is near convergence. It is possible that the initial condition information will have to be modified to allow for a more adequately spun-up eddy field. Still, this field, along with the northern boundary condition controls and the atmospheric state controls will be useful. They will contain the large scale information necessary to allow the model to reproduce the observed sea surface height and meridional mass, heat, and freshwater flux. It is also important to remember that the adjoint model is forced by the model-data misfit. Starting with a more converged solution results in weaker forcing, and thus reduces the chance of adjoint model instabilities. In a highly non-linear low viscosity model, one must be wary of the optimization stalling due to cost function local minima. Beginning at an initial state relatively close to an acceptable minimum is extremely desirable.

Non-linear models often have rough and highly variable cost functions and this increases the chances of the optimization stalling (Gebbie; 2004). The chance of the cost function stalling can be reduced by emphasizing the large scale state. This is most rigorously achieved by providing the error covariances in the weighting matrices. Unfortunately, error covariances have yet to be estimated for the controls and observations being used. Furthermore, were these covariances available, the current cost function numerical code would need to be modified. The implementation of error covariances will be deferred for future work. An attempt to approximate them can be made, however, by the implementation of a smoothness constraint for the controls. This smoothness constraint is a penalty placed on the magnitude of the Laplacian of the atmospheric state and the initial condition control vectors (see equation (2.1r). 
Forcing a smooth control field constrains the optimization to the physically reasonable assumption that the error in neighboring model input points is of similar magnitude and direction.

\subsection{Gradient Descent}

A review of optimization methods is far beyond the scope of this thesis. There is a substantial literature on optimization theory (see (Gill et al.; 1986) for example ). It is worth noting, however, that this optimization problem of considerable dimension is made possible because the Lagrange multiplier method employed makes known the gradients of the cost function with respect to the controls $(\partial J / \partial \mathbf{u}(t))$. Knowledge of these gradients increases the efficiency of search algorithms. Research has shown quasi-Newtonian optimization algorithms to be superior to other optimization algorithms for the sort of non-linear ocean optimization at hand because these methods account for the curvature of the cost function(Gebbie; 2004). Quasi-newtonian algorithms are more efficient than steepest descent algorithms at avoiding local minimas (Press et al.; 1992). The gradient search method of Gilbert and Lemaréchal (1989) uses stored gradients of the cost function from previous iterations to approximate the Hessian matrix. By only approximating the Hessian matrix, valuable second derivative (curvature) information is made available without an excessive computational demand. 


\section{Chapter 4}

\section{Observations and Uncertainties}

\subsection{Observations}

\subsubsection{Altimetry}

Satellite altimeters, which provide the most observations in the year 2000, are used to constrain both the model's mean and time-varying component of sea surface height (SSH). The separation of these two SSH components is made in order to distinguish errors associated with the geoid from those due to the time-evolving dynamics. The model is constrained to the mean ocean dynamic topography relative to the Gravity Recovery and Climate Experiment (GRACE) project geoid. This data set is processed and provided by the Physical Oceanography Distributed Active Archive Center (PODAAC) at the Jet Propulsion Laboratory. More information on this data set and its processing is available at http://podaac.jpl.nasa.gov/.

The model sea surface height anomaly is constrained by data from the European Remote-Sensing Satellites (ERS-2), and data from the the Topex/Poseidon (T/P) satellite. The ERS-2 satellite provides coverage for the full state estimate spatial domain, and the $\mathrm{T} / \mathrm{P}$ satellite has coverage equatorward of $\sim 67^{\circ} S$. The ERS-2 and $\mathrm{T} / \mathrm{P}$ data are processed and provided by Aviso (http://www.aviso.oceanobs.com/) and PODAAC respectively. The along orbital track data provided is binned into the state estimate grid for each day of the simulation; no alteration or interpolation of 
the data is performed. The model sea surface height is averaged daily, the time mean is subtracted off, and the resulting field is compared to the SSH anomaly observations data set as cost function terms (2.1d) on page 20 .

\subsubsection{Climatology}

From the surface to 300 meters the model state is constrained to the monthly hydrographic climatology obtained from the NOAA World Ocean Atlas 2000 (Levitus et al.; 2001; Conkright et al.; 2002). At depths below 300 meters the model is constrained to the temporally constant climatology of Gouretski and Koltermann (2004). These temperature and salinity climatologies are interpolated to the model grid and compared to the model state climatology. (The in situ temperatures given in the climatologies are converted to potential temperatures for comparison with the model state.)

\subsubsection{CTD and XBT In Situ Data}

CTD (conductivity, temperature, and depth) and XBT (expendable bathythermograph) in situ data were bin-averaged to the model $1 / 6^{\circ}$ grid for each month of the state estimate. There are 536 CTD temperature and salinity observations and 11,943 XBT observations in the state estimate domain for the year 2000. In situ temperature observations were converted to potential temperature for comparison with the model state. It must be noted that Argo float profiles are present in the Southern Ocean only after July 2001. The number of observations taken by these autonomous floats increases dramatically after this date. The fact that there is no Argo data in 2000 is an unfortunate oversight that was made when the optimization time interval was chosen.

\subsubsection{Sea Surface Temperature}

The model is constrained by mean sea surface temperature (SST) fields provided by Reynolds, Rayner, and Smith (2002). This data set, which accounts for sea ice 
cover, is a combination of in situ and satellite observations. More information can be found at http://www.emc.ncep.noaa.gov/research/cmb/sst_analysis/. The SST data is acquired as monthly fields and then interpolated to the model grid.

\subsection{Uncertainty}

The following two sections, Section (4.3) and Section (4.4), explain the choice of error covariance matrices ( $\mathbf{Q}$ and $\mathbf{W}$ of Equation (2.2)) used in this work. The oceanographic community has not yet come to agreement on procedures for determining these matrices, and the ECCO group has thus far only estimated the diagonal terms. In this work, all non-diagonal terms have been set to zero. (As discussed in Section 3.3, the smoothness constraint imposed on the control vectors does imply a level of error covariance.)

The weighting matrices used represent a combination of both model and observational uncertainty. Observational uncertainty arises from instrument noise and noise introduced by the removal of processes not being modeled. Model uncertainty, which is the error expected in the model-data misfit were the data perfect, arises due to missing small scale dynamics owing to a lack of resolution. For example, boundary current transport may be correctly estimated, but if the boundary layer is not resolved, the flow structure near this boundary will be incorrect. For this reason, it is to be expected that the model state will be further from observations in highlyenergetic western boundary current regions. In other words, the acceptable misfit of an XBT temperature profile in the Agulhas Current should be larger than in the relatively quiescent Brazil Basin. Model representation error should become less significant as resolution is increased.

The degree of representation error in the simulation at hand is unknown, and so it is important to note that the uncertainty fields used are estimates. Estimating error covariance matrices is a difficult and time consuming task of great importance. The state estimate is no better than the choice of these matrices as the weighting of the cost function determines the solution. If the error is underestimated, the model 
is constrained to noise; if the error is overestimated, useful information is discarded. Unfortunately, like the Southern Ocean's role in the global climate, there is a gap between significance and knowledge. Much work is still needed in determining the misfit expected when fitting modern ocean models to observations.

The weighting matrices used in this work are ever evolving; if a constraint appears to be too loose or too tight, the uncertainty estimate will be evaluated and, if thought appropriate, altered. The current weighting matrices used in the state estimation are given below. The reader should remember that error due to model representation of the observations is included in the weighting matrices of each data source.

\subsection{Estimated Uncertainty in the Observations}

All the uncertainty estimates used to constrain the model to the observations are adopted from the ECCO-GODAE $1^{\circ}$ resolution global state estimate. One is referred to this work (see for example Lu et al. (2002) or Wunsch and Heimbach (2005b)) for a supplemental accounting of the fields.

\subsubsection{Altimetry}

\section{Time-Varying Sea Surface Height Anomaly}

A comparative analysis between the $\mathrm{T} / \mathrm{P}$ and Jason-1 altimeters during their "tandem" orbits by Ponte, Wunsch, and Stammer (2005), hereafter PWS, found the measurement errors ranged from $\sim 2 \mathrm{~cm}$ in the tropics to $\sim 4 \mathrm{~cm}$ in the high latitudes. They found altimeter errors to be strongly dependent on significant wave height amplitudes; a finding especially important (and unfortunate) for this study as the significant wave height in the Southern Ocean is relatively high, often on the order of 10 meters.

PWS derive two spatially varying global SSH observational uncertainty estimates. The first is derived by combining tropospheric uncertainties estimated from differences in atmospheric reanalysis products (these errors are on the order of $0.5 \mathrm{~cm}$ ) with the 
root mean squared differences between $\mathrm{T} / \mathrm{P}$ and Jason-1 tandem mission data. They note that this estimate should be a lower bound as it omits errors common to the altimeter observations and the re-analysis products.

A globally averaged uncertainty budget for $\mathrm{T} / \mathrm{P}$ is summarized in Chelton et al.(2001). PWS derive their second error estimate by adding the globally averaged wet tropospheric uncertainty, ionospheric uncertainty, and orbital uncertainty from Chelton et al. $2001(1.1 \mathrm{~cm}+0.5 \mathrm{~cm}+2.5 \mathrm{~cm}=4.1 \mathrm{~cm})$ to the uncertainty from radar error and electromagnetic and skewness bias that they parameterize as being proportional to the standard deviation of the significant wave height, $\sim 1.02 \sigma_{s w h}$. The two error maps PWS calculated were in reasonable agreement, with the latter method showing a greater tendency for large uncertainty values.

Uncertainty arises from the removal of both ocean tides and atmospheric pressuredriven signals from the altimetric observations. In the analysis carried out by PWS for the ECCO-GODAE state estimate, $1 \mathrm{~cm}$ was added to the representation error for deep-sea tides. An uncertainty for pressure-driven signals was added using a simple inverted barometer relation of $\sim 1 \mathrm{~cm} / \mathrm{hPa}$. A lower bound for the error in the atmospheric sea level pressure field was derived by taking the standard deviation of the differences between the European Centre for Medium-Range Weather Forecasts (ECMWF) and NCEP pressure re-analysis fields.

Model representation uncertainty was calculated by comparing the variance in the ECCO-GODAE $1^{\circ}$ state estimate to that of a forward model with $1 / 8^{\circ}$ resolution. It was found that in highly energetic regions such as the ACC, the SSH anomaly uncertainty budget is dominated by the representation error due to unresolved mesoscale activity.

The final uncertainty field produced for the ECCO-GODAE state estimate is a combination of the observational error, the processing error, and the representation error components described above. The observational error field used is the one PWS derived using the standard deviation of the significant wave height (the second method described above). In quiet ocean regions, these three error components contribute comparably. In energetic regions the contribution from representation error 
dominates.

For this project, the sea surface height anomaly uncertainty field determined by PWS is interpolated to cover the model domain. Regions with extremely sparse data coverage have been set to the maximum uncertainty value of $\sim 36.3 \mathrm{~cm}$. The median uncertainty value in the domain is $\sim 10.3 \mathrm{~cm}$.

\section{Mean Sea Surface Topography}

The mean sea surface height error file used in the ECCO-GODAE production run is interpolated to the model grid. The dominant contribution to the field comes from taking $1 \%$ of the significant wave height field provided by Chelton et al.(2001). Instrument error, which is expected to be about $1 \mathrm{~cm}$, is included in the estimated error. A representation error of another centimeter is added. Relatively small contributions from the mean variance in $\mathrm{T} / \mathrm{P}$ and from the mean inverted barometer effect are also included. In the Southern Ocean this uncertainty field ranges from $\sim 3.1 \mathrm{~cm}$ to $\sim 5.6 \mathrm{~cm}$. Where uncertainty data was absent (e.g. the Ross Sea) the error was set to the maximum expected GRACE geoid error of $\sim 5.6 \mathrm{~cm}$. The median uncertainty is $\sim 4.7 \mathrm{~cm}$. To ensure no sharp contrasts in the uncertainty field a gaussian smoothing is applied to the field.

\subsubsection{In Situ Data}

As part of the ECCO consortium, Gaël Forget has determined a spatially varying uncertainty field for the in situ hydrographic data (Forget and Wunsch; 2006). The field is a climatological error in the sense that it is based on binning the data and examining the standard deviation of the error within the bins. This assumption of stationary ocean variability is necessary due to scarcity of data. The mean seasonal cycle was accounted for, however, in the top 1000 meters. An extrapolation mapping technique (Rhein et al.; 2002) was necessary where data were too scarce for a standard deviation to be calculated. This mapping method was used as a smoother where data were more abundant. An uncertainty field was produced from standard deviations 
provided by Levitus et al.(2001), and from the standard deviations calculated by Forget. Forget's analysis focused on recent CTD and Argo data, which were absent in the calculation of Levitus et al.(2001). The vertical structure of the two uncertainty fields is consistent. This is reassuring as the Levitus et al. vertical uncertainty profile is used to weight the open boundary condition control vectors. The two uncertainty fields are averaged, and then the mapping is re-applied to slightly smooth the solution. The derived uncertainty estimate suggests observational variability on par with a $1 / 8^{\circ}$ resolution forward model run, and thus representative error in this estimate is of acceptable magnitude. As one would expect, the uncertainty field has greatest magnitudes in the highly energetic regions.

The uncertainty field was interpolated to the $1 / 6^{\circ}$ model grid and smoothed. Uncertainty values for temperature below $.05^{\circ} \mathrm{C}$ and below .01 for salinity are replaced by the vertical uncertainty profile values derived by Levitus et al. The median (in the horizontal) of the uncertainty field used is plotted in Figure (4-1).

\subsubsection{Climatology}

The estimated climatological uncertainty used in the ECCO-GODAE state estimate is derived by Gaël Forget. This field must represent the error from instrument noise and representation errors. The field must also include errors introduced by climatic variability, as the model does not cover the full period of time the climatology represents. The spatially varying error estimate provided by Gouretski and Koltermann (2004) is used for the noise and representation errors. An uncertainty for climatic variability is included which allows the model to deviate from the climatology to a degree on par with the overall climatic variability. The field Forget derived is interpolated to the $1 / 6^{\circ}$ and smoothed using a Gaussian filter. The median (in the horizontal) of the climatological uncertainty field is plotted in Figure (4-1). 


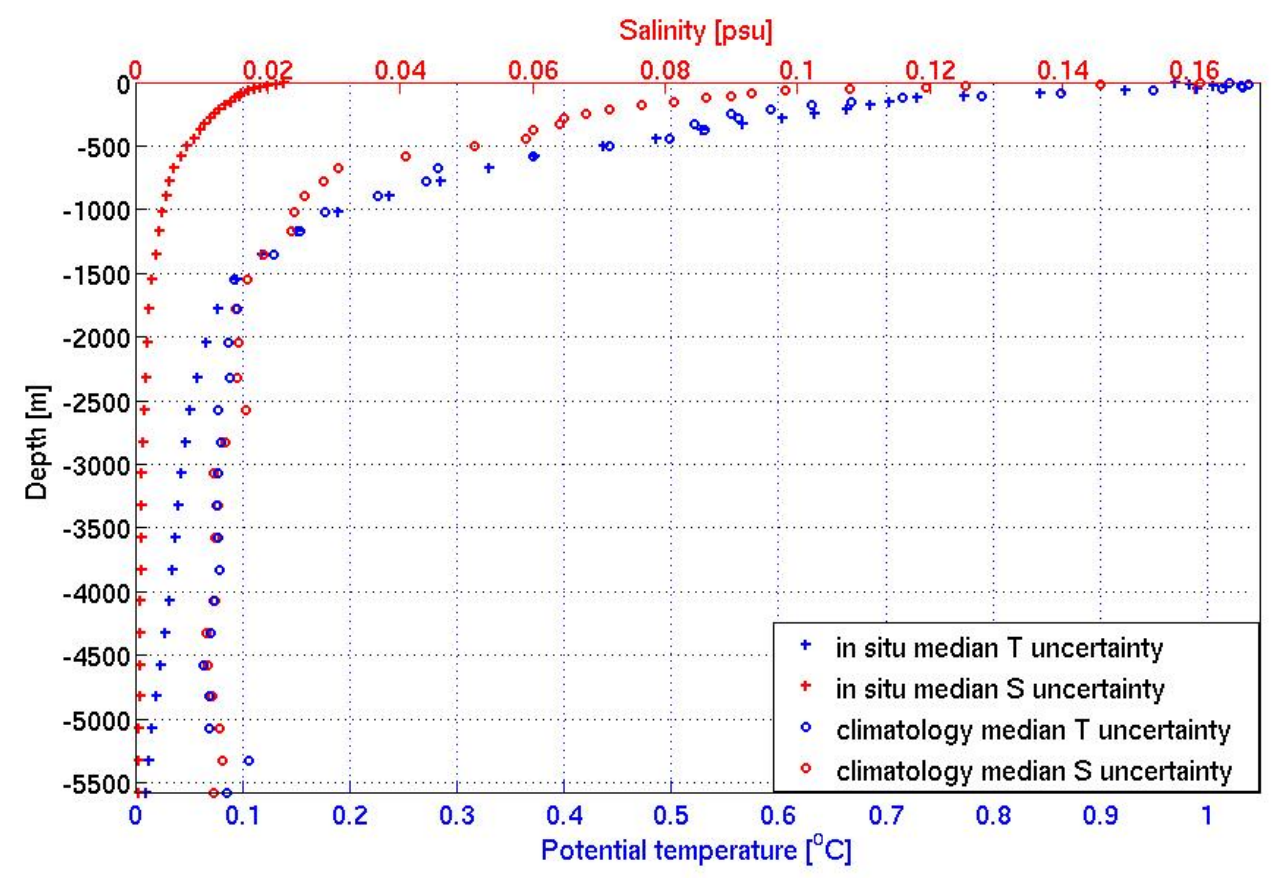

Figure 4-1: Median uncertainty (calculated horizontally over the domain) for the in situ observations and hydrographic climotology.

\subsubsection{Sea Surface Temperature}

A constant uncertainty value of $2.8^{\circ} \mathrm{C}$ has been assigned to this field. This represents a considerable increase from the $\sim 0.5^{\circ} \mathrm{C}$ value used in the ECCO-GODAE production run, and was chosen to account for both interpolation errors and for the smoothness of processed sea surface temperature field.

\subsection{Estimated Uncertainty in the Controls}

The control vectors are the perturbations to the initial conditions, the northern boundary conditions, and the prescribed atmospheric state. The first estimate used for the control values is zero. The optimization procedure determines values for these vectors that minimize the cost function. The controls, however, are constrained to remain within the uncertainty of the fields they represent. The uncertainties attributed 
to the controlled fields are described below. The atmospheric state controls and the northern boundary controls are linearly interpolated between values given at 10 day intervals. All the control vectors have full spatial resolution.

\subsubsection{Prescribed Atmospheric State Uncertainty}

As explained in Section (3.2.2), an initial guess of the atmospheric state is taken from the NCEP re-analysis fields. Control vectors are used to perturb this estimated state. The time-mean uncertainty is given as a constant value. The values used, chosen from experience with the ECCO $1^{\circ}$ bulk formula state estimate, are given in Table (4.1).

The uncertainty in the time-varying NCEP prescribed atmospheric state are derived from the temporal standard deviations of the fields from 1992 to 2003. These standard deviations are questionably small over large regions of the domain. To remedy this, any value that was less than $50 \%$ of the maximum standard deviation in the domain was increased to this larger value. The fields were then all smoothed with a Gaussian filter. Table (4.2) gives the minimum, median, and maximum uncertainty values for the time-variable component of the prescribed atmospheric state

\begin{tabular}{|l|l|}
\hline \multicolumn{2}{|l|}{ Atmospheric state uncertainty estimate (time mean component) } \\
\hline Air temperature & $4^{o}$ Celsius \\
\hline Specific humidity & $2 \times 10^{-3}$ kilogram water vapor / kilogram air $^{-1}$ \\
\hline Meridional wind speed & 4 meters second \\
\hline Zonal wind speed & 4 meters second $^{-1}$ \\
\hline Precipitation & $6 \times 10^{-8}$ meters second $^{-1}$ \\
\hline Short wave radiative flux & 60 watts meter \\
\hline
\end{tabular}

Table 4.1: Uncertainty in the time mean component of the NCEP prescribed atmospheric state 


\begin{tabular}{|l|c|c|c|l|}
\hline \multicolumn{5}{|c|}{ Atmospheric state uncertainty estimate (time variable component) } \\
\hline Atmospheric field & minimum & median & maximum & units \\
\hline Air temperature & 10 & 10 & 20 & degrees Celcius \\
\hline Specific humidity & 1.7 & 1.7 & 3.5 & $10^{-3} \mathrm{~kg} \mathrm{H}_{2} \mathrm{O}(\mathrm{kg} \text { air })^{-1}$ \\
\hline Meridional wind speed & 2.7 & 4.3 & 5.4 & meters second $^{-1}$ \\
\hline Zonal wind speed & 3.3 & 4.6 & 6.6 & meters second $^{-1}$ \\
\hline Precipitation & 4.4 & 4.4 & 8.7 & $10^{-8}$ meters second $^{-1}$ \\
\hline Short wave radiative flux & 55 & 74 & 110 & watts meter $^{-2}$ \\
\hline
\end{tabular}

Table 4.2: Minimum, median, and maximum uncertainty in the time variable component of the NCEP prescribed atmospheric state

\subsubsection{Initial Condition Uncertainty}

A one year spin up from the ECCO-GODAE production run (iteration 163) is used as the first guess initial conditions. The model configuration used in this project is quite different from the ECCO-GODAE state estimate, and it is possible that the interpolation and spin-up applied may have resulted in an initial condition field rather far from the optimal state. The uncertainty used to constrain the model to the climatology (see Section 4.3.3) is used to weight the initial conditions. As this field may have large errors, the uncertainty was increased by a factor of 20 from the climatology uncertainty field.

\subsubsection{Open Northern Boundary Condition Uncertainty}

A longitudinally constant vertical profile is used to specify the uncertainty in the open boundary condition. For the prescribed temperature and salinity field, this uncertainty profile was derived from the standard deviations provided from Levitus et al.(2001). (This profile was found to be consistent with one calculated from recent CTD and Argo data by Forget, see Section 4.3.2). The northern boundary condition, applied at $\sim 24.7^{\circ} \mathrm{S}$, is taken from the ECCO-GODAE production run (iteration 163). The error field being used to constrain the open boundary is derived as an uncertainty 
estimate for the hydrographic climatology; using it implies that the uncertainty in the ECCO-GODAE $1^{\circ}$ state estimate is approximately equal to that of the climatology. This may seem to be an overestimate, however considering representation error between the Southern Ocean state estimate and the interpolated ECCO field, this error bound is reasonable.

The observed surface geostrophic velocity at the northern boundary is calculated from the sea surface height field given by the $\mathrm{T} / \mathrm{P}$ altimeter. This field is compared to the surface geostrophic velocity as calculated from the ECCO-GODAE production run sea surface height. The variance of the misfit between these two fields is calculated. The assumption is then made that half this variance can be explained by the barotropic velocity squared, and half by the fist baroclinic mode surface velocity squared. (The average buoyancy frequency from the region is used to determine the vertical mode decomposition.) Using these two vertical modes, an error profile can be extrapolated to depth. This profile is averaged longitudinally. The derived vertically varying uncertainty profile is a good estimate over much of the region, but it is probable too small in boundary current regions. In the future it would be beneficial to loosen this constraint by increasing the error in energetic regions. For now it is assumed that a reasonable lower bounds on the error has been obtained. 


\section{Chapter 5}

\section{Production of a Southern Ocean State Estimate}

Two goals were set for this thesis. The first goal was to determine if a large-scale eddy-permitting Southern Ocean ocean model could be brought into consistency with observations. Proof of feasibility results in the production of a Southern Ocean state estimate. With the first goal accomplished, one can address the second goal of the thesis, which is to use the state estimate to shed light on the climate and dynamics of the Southern Ocean.

The first goal has been accomplished; the production of a high resolution Southern Ocean state estimate is feasible. Work carried out, which allows this conclusion to be drawn, is described in Section 5.1. This section discusses the performance of the optimization, and shows that the model-data misfit was considerably reduced. In Section 5.2 the consistency of the state estimate with observations is discussed. It is found that the state estimate solution at hand has yet to converge to what is deemed an acceptable solution. Nonetheless, it is worthwhile to pause the optimization and analyze the current (preliminary) state estimate.

The state estimate is a solution to a high-resolution state-of-the-art numerical model. Much of what is known of the Southern Ocean comes from similar models (e.g. FRAM and OCCAM). Analysis of the model solution produced in this work has a large advantage over past model analysis. The state estimate solution that has been 
produced has been compared to observations in detail. Inferences drawn from this solution may therefore be tested. Section 5.2 points out the strengths and weaknesses of the state estimate solution.

\subsection{Feasibility of Eddy-Permitting Southern Ocean State Estimation}

Ocean state estimation is extremely computationally intensive. To run a global state estimate on a single processor computer one would be limited to a horizontal resolution of approximately $4^{\circ}$. To approach resolutions considered eddy-permitting, a supercomputer is absolutely necessary. The Southern Ocean state estimate presented, though regional, still covers roughly a third of the globe, and so acquiring supercomputing resources was a necessary step.

Besides simply acquiring massive computers to push through massive computations, one must work to ensure the efficiency of the computation at hand. The ECCO group has spent considerable time testing and optimizing the MITgem forward and adjoint model on a variety of platforms. One of the greatest impediments to the feasibility of large state estimates is efficiency reading to, and writing from, storage devices during computations (known as input/output, I/O). Customization of the code allows one to maximize stored variables and minimize I/O. This project represents the state estimate with the largest grid ever carried out by the ECCO consortium. The beginning of this project consisted of a large effort, led by Patrick Heimbach, aimed at maximizing the efficiency of carrying out this state estimate (and this effort continues). Many new I/O routines were added to the MITgcm that will aid this project, and future large scale state estimates. Routines were also optimized to minimize the amount of local storage necessary. State estimation using the adjoint method remains, however, significantly restricted by the amount of local memory available per processor. 
The MIT ECCO group received an invitation to test the project at San Diego Super Computing Center's (SDSC) new 32GB, 8 processor nodes on their DataStar cluster. DataStar demonstrated excellent I/O performance. It was determined that running a $1 / 6^{\circ}$ state estimate on 600 processors at SDSC would be feasible; each iteration would take about 48 computer hours. It's worth noting that the 600 processor Southern Ocean state estimate runs at comparable speeds to the 60 processor ECCOGODAE production run set-up. That both the forward and the adjoint components scale well is a considerable feat of software and hardware engineering giving great promise for next generation ECCO projects.

A proposal to SDSC for computer time (beyond the test period) was submitted in January 2006, and accepted in March ensuring that this $1 / 6^{\circ}$ resolution Southern Ocean state estimate could be attempted. In the Southern Ocean, the decorrelation scale for spatial variability is on the order of $85 \mathrm{~km}$ (Gille; 1995), and the Rossby deformation radius is on the order of $18 \mathrm{~km}$ (Marshall et al.; 1993). A model resolution of $1 / 6^{\circ}$ (18.5 km meridionally, and $3.9 \mathrm{~km}$ to $16.8 \mathrm{~km}$ zonally) can be considered "eddy-permitting", as larger eddies are resolved. For a truly eddy resolving simulation, a model must resolve higher order derivatives on the scale of the deformation radius.

A $1 / 6^{\circ}$ forward model was designed (Section 3.1), and run, for the year 2000. Best guess initial and northern boundary condition were derived from the $1^{\circ}$ ECCO global ocean state estimate (Section 3.2). Output from the model was compared to observations, and found to be qualitatively consistent. A cost function was then designed (Section 2.2) to quantify the model misfit from the observations. Model control variables were identified (Section 2.3 and 4.4). Using the adjoint method (Section 2.3 to 2.5 ), the gradient of the cost function with respect to the controls was obtained. Perturbations to the controls were found based on these gradients (Section 3.4). At the time of the writing of this thesis, eight iterations (each consisting of running the forward model, calculating the cost, running the adjoint model to calculate the cost function gradients, and then updating the control vectors) had been carried out. The model-observation misfit was reduced over the eight iterations. 


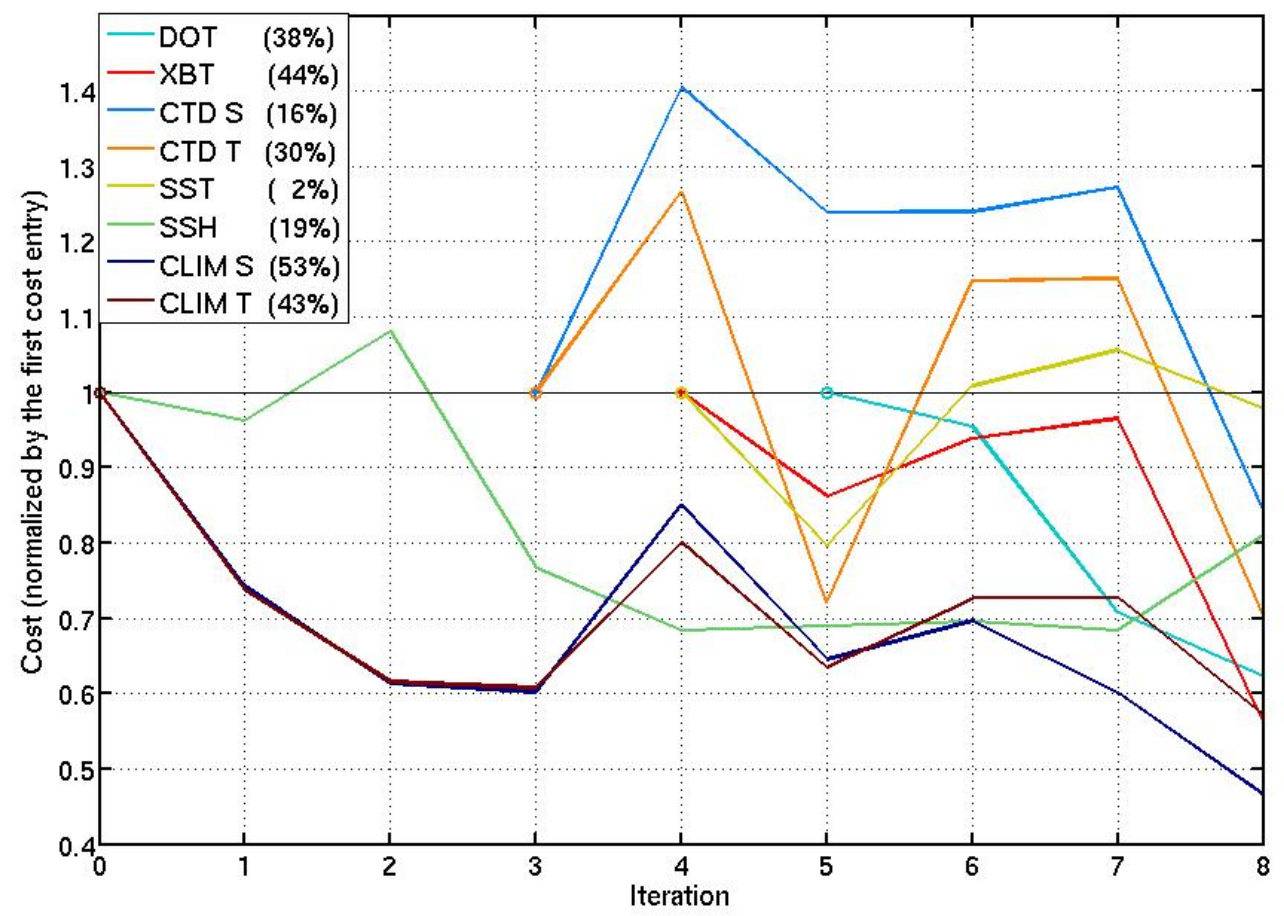

Figure 5-1: Cost terms due to model state misfit from observations. These terms are normalized by the first cost value so that the performance of the adjoint method can be deduced. The percent decrease from first cost entry to iteration 8 is shown in the legend. See Section 4.1 for a description of the observations and Section 4.3 for a description of their weights.

The machinery employed to reduce the model state's misfit from observations is working. Figure (5-1) plots the cost of the misfit to the observations for each iteration. Cost terms were added as the state estimate ran, so each cost term has its own starting iteration. The cost terms have been divided by their starting value. While this masks the magnitudes of the costs, and thus hides the adequacy of the model-observation fit, it does allow evaluation of the performance of the state estimate. (The actual misift to observations of the state estimate (iteration 8) is evaluated in Section 5.2.)

Figure (5-1) shows that every cost term has been reduced. The reduction for each term, from its initial inclusion to iteration 8, is given in the figure legend. The adjoint method works to reduce the total cost. The optimization will bring the average misfit down, which means some individual cost terms may increase. It is acceptable for one observational data set cost to increase, if other terms decrease. At the final converged 
state, however, all cost terms should be reduced to an acceptable level. At the current stage of the state estimate, some cost are still increasing from iteration to iteration. For example, the sea surface height anamoly (SSH) cost has increased since iteration 4, likely owing to the fact that the optimization focused its efforts on the many other cost terms present. The optimization appears to be working very well at reducing the misfit to the mean sea surface height (DOT). This cost term has come down by $38 \%$ over just 3 iterations.

Though the total cost has come down $84 \%$ since the optimization began, iteration 8 has yet to reach an acceptable state. The downward trend of Figure (5-1) suggests, however, that the cost will continue to be reduced as the state estimate continues. Bringing a $1 / 6^{o}$ eddy-permitting Southern Ocean model into consistency with observations is a feasible, though demanding, exercise.

\subsection{Model-Observation Misfit}

Though the initial model run used what was arguably the most realistic boundary and initial conditions available, the state it produced was not fully consistent with observations. Using the adjoint model, this state has been brought considerably closer to observations (see Section 5.1 above). The state estimate is currently consistent with observations in some regions, and inconsistent in others. In this section the performance of the state estimate in fitting the observations is evaluated.

The goal of the state estimate is to bring the model state into consistency with observations. If each observation were independent (this is not the case for the climatology due to its processing), consistency would be defined such that the mean of the misfit would approach zero, and the variance of the normalized misfit (misfit magnitude divided by uncertainty) would approach one. The cost, which is the normalized misfit squared, should then have a $\chi^{2}$ distribution. A $\chi^{2}$ distribution is plotted in Figure (5-2). From this figure it can be seen that while $\sim 68 \%$ of the cost values

should be less than one, it is to be expected that an acceptable solution will have a small percentage of cost values far greater than one. (The $\chi^{2}$ distribution plotted 
assumes 1 degree of freedom implying that there is one independent state variable, $x_{i}$, to fit each observation, $y_{i}$.)

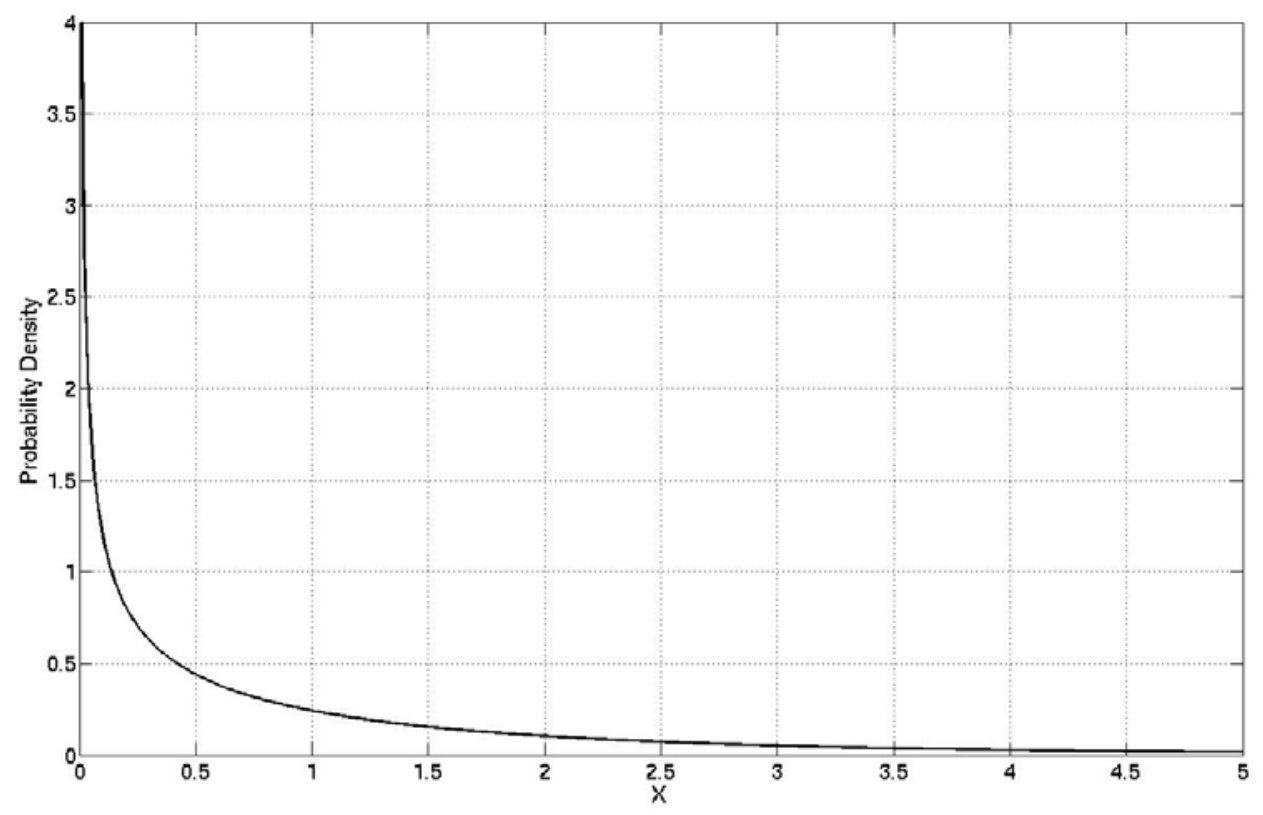

Figure 5-2: Chi-square probability density function.

In the following subsections, the model misfit to climatology and altimetry is evaluated. Plots of model misfit to XBT and CTD in situ data are not shown. The $\mathrm{XBT}$ data exists primarily equatorward of $40^{\circ} \mathrm{S}$ and at depths above 3000 meters. On average, the model is fairly consistent with the XBT data; the average cost per term is 2.5. The CTD data, which are far more sparse, are not fit as well by the model; the mean cost per term is 21.5 for temperature observations and 11.0 for salinity observations. The uncertainty assigned to the sea surface temperature data requires greater analysis; at present a constant tolerance of $2.8^{\circ} \mathrm{C}$ is allowed. Analysis of the sea surface temperature data, and the model misfit to this data, is left for future work. 


\subsubsection{Climatology}

\section{Temperature}

Figure (5-3) shows the misfit of the model state to the ocean temperature climatology. Panel A shows that compared to the climatology, the model state has a slight cooling trend near Antarctica, and a slight warming trend equatorward of $60^{\circ} \mathrm{S}$. The climatology data are smooth, and therefore one may expect large scale patterns in the misfit. Panels B and C do show large patterns, but they are smaller than individual ocean basins, suggesting no full-basin heat-content misfit tendency is present in the model. Panel D shows that there is a misfit dependence on latitude. On average, the surface waters are $\sim 1^{\circ} \mathrm{C}$ cooler than the climatology equatorward of $50^{\circ} \mathrm{S}$, which is an acceptable misfit. The model surface waters are $\sim 1^{\circ} \mathrm{C}$ warmer than the climatology poleward ward of this latitude, and this misfit is greater than the uncertainty prescribed to the climatology. (The uncertainty in the climatology is discussed in Section 4.3.3.) The misfit of the intermediate waters have the opposite misfit signature, warmer to the north, colder to the south. Taking into account climatological uncertainty, the intermediate water misfit is acceptable in the northern part of the domain, but poleward of $\sim 60^{\circ} \mathrm{S}$ the misfit is too large. At depths below 2,000 meters the model state is within $0.5^{\circ} \mathrm{C}$ of the climatology. A $0.5^{\circ} \mathrm{C}$ misfit is considered acceptable above $\sim 2,500$ meters, but below this depth is outside of the climatological uncertainty.

Figure (5-4) shows the cost associated with the climatology misfit. As explained above, a cost greater than 1 implies the model state is outside the uncertainty bounds placed on the climatology. This uncertainty is greater than $1^{\circ} \mathrm{C}$ at depths less then 1,000 meters and latitudes equatorward of $\sim 60^{\circ} \mathrm{S}$. For this reason, the solution is for the most part consistent with the climatology northwards of the Polar Front. Above 1,000 meters and poleward of $\sim 60^{\circ} \mathrm{S}$ the uncertainty is order $0.5^{\circ} \mathrm{C}$, and thus there is a large cost associated with the order $1^{\circ} \mathrm{C}$ misfit in this region. A large cost is also found below 3000 meters where the uncertainty in the climatology is only expected to be $\sim 0.2^{\circ} \mathrm{C}$. 

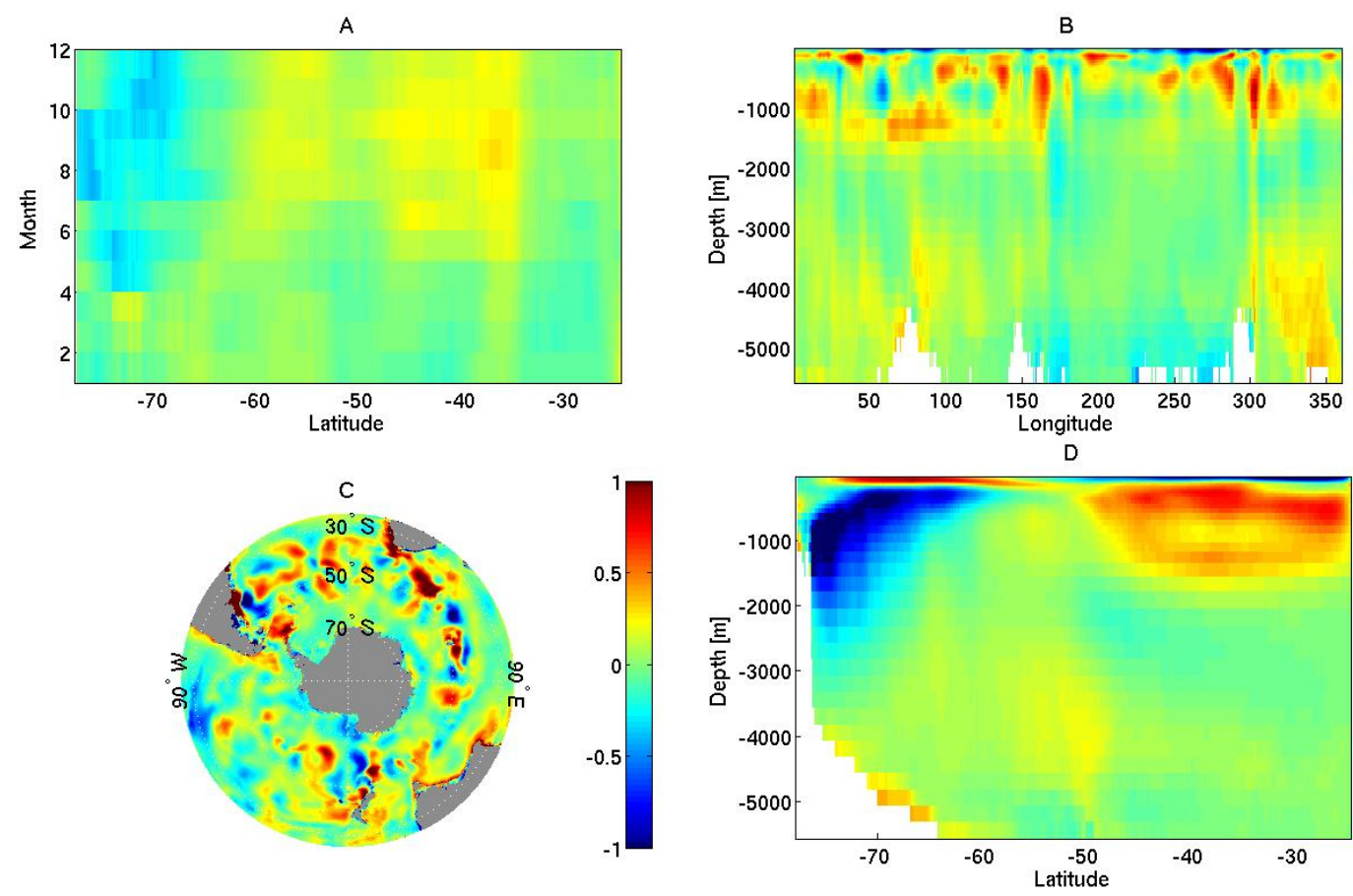

Figure 5-3: Model monthly temperature means minus climatology (positive values denote the model state is warmer than the climatology). Units are potential temperature $\left[{ }^{\circ} \mathrm{C}\right]$. A) Latitude vs. month (zonally and vertically averaged). B) Longitude vs. depth (latitudinally and temporally averaged). C) Longitude vs latitude (vertically and temporally averaged). D) Latitude vs. depth (longitudinally and temporally averaged).
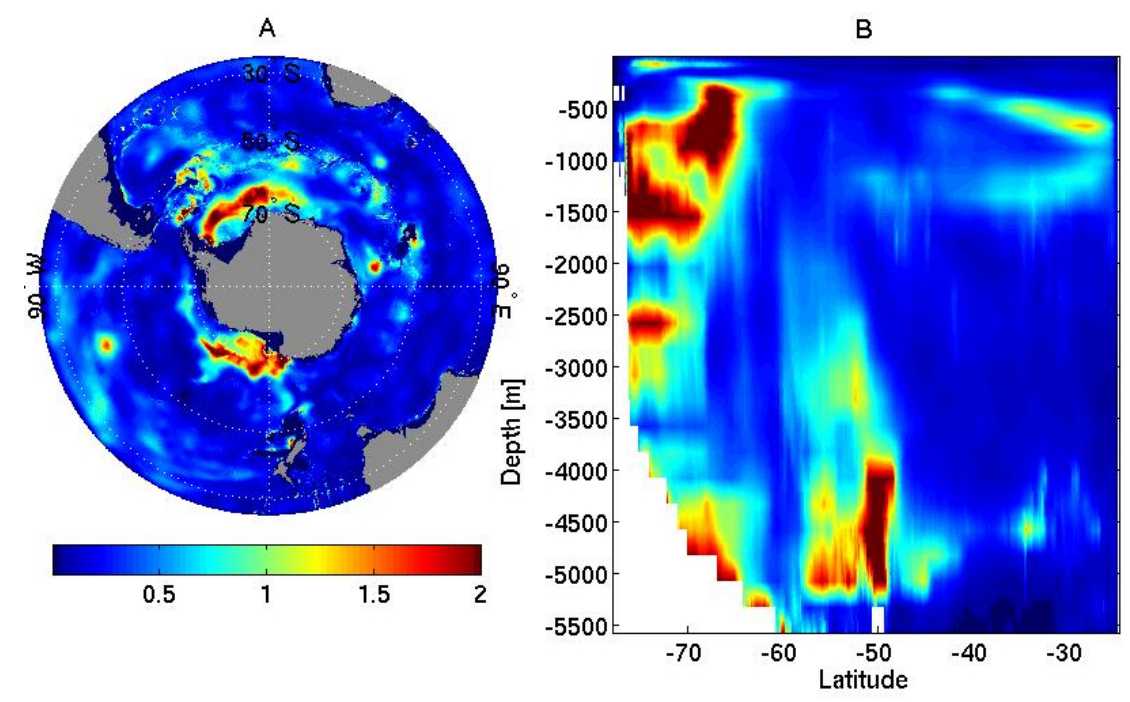

Figure 5-4: Cost associated with misfit shown in Figure (5-3). The cost equals the weighted (by uncertainty) misfit squared. Panels A and B are analogous to Panels C and D of Figure (5-3). 


\section{Salinity}

Figure (5-5) shows the misfit of the model state to the ocean salinity climatology. Panel A shows a small freshening trend in the model at latitudes equatorward of the Subantarctic front. Panel B shows that, in contrast to the temperature misfit, the salinity misfit is rather constant in longitude. Panel $\mathrm{C}$ shows the misfit to be dependent on latitude. Panel D shows that, near the surface, the model state is saltier than the climatology poleward of $50^{\circ} \mathrm{S}$, and fresher than the climatology equatorward of this latitude. The opposite pattern is shown for the intermediate water. Comparing this plot to Panel D of Figure (5-3), it can be seen that salinity and temperature differences compensate such that the model density structure is likely to be similar to that of the climatology (i.e. the model is either warmer and saltier, or colder and fresher, than the climatology).

Figure (5-6) shows the cost associated with the salinity misfit. The model state is largely consistent with the climatology at depths below 2,000 meters. At shallower depths the model state is rather consistent in the Antarctic Circumpolar Current region, but deviates north and south of these latitudes. Similar to the temperature cost, there is still much improvement needed to bring the model into consistency with the climatology near the Ross and Weddell Seas.

Sparsity of observations was accounted for in determining the uncertainty in the climatology. Nonetheless, one is most distrustful of the climatology in the poorly sampled Subpolar Zone. Panel B of Figures (5-4) and (5-6) show that this is the very region where the state estimate is least acceptable with respect to the climatology. Future work should include a reevaluation of the climatological uncertainty in this region. It is very possible that variability (for example in the strength of the Ross and Weddell Sea Gyres, or in the atmospheric forcing) causes a larger uncertainty in the climatology along the Antarctic Shelf than was previously realized. 

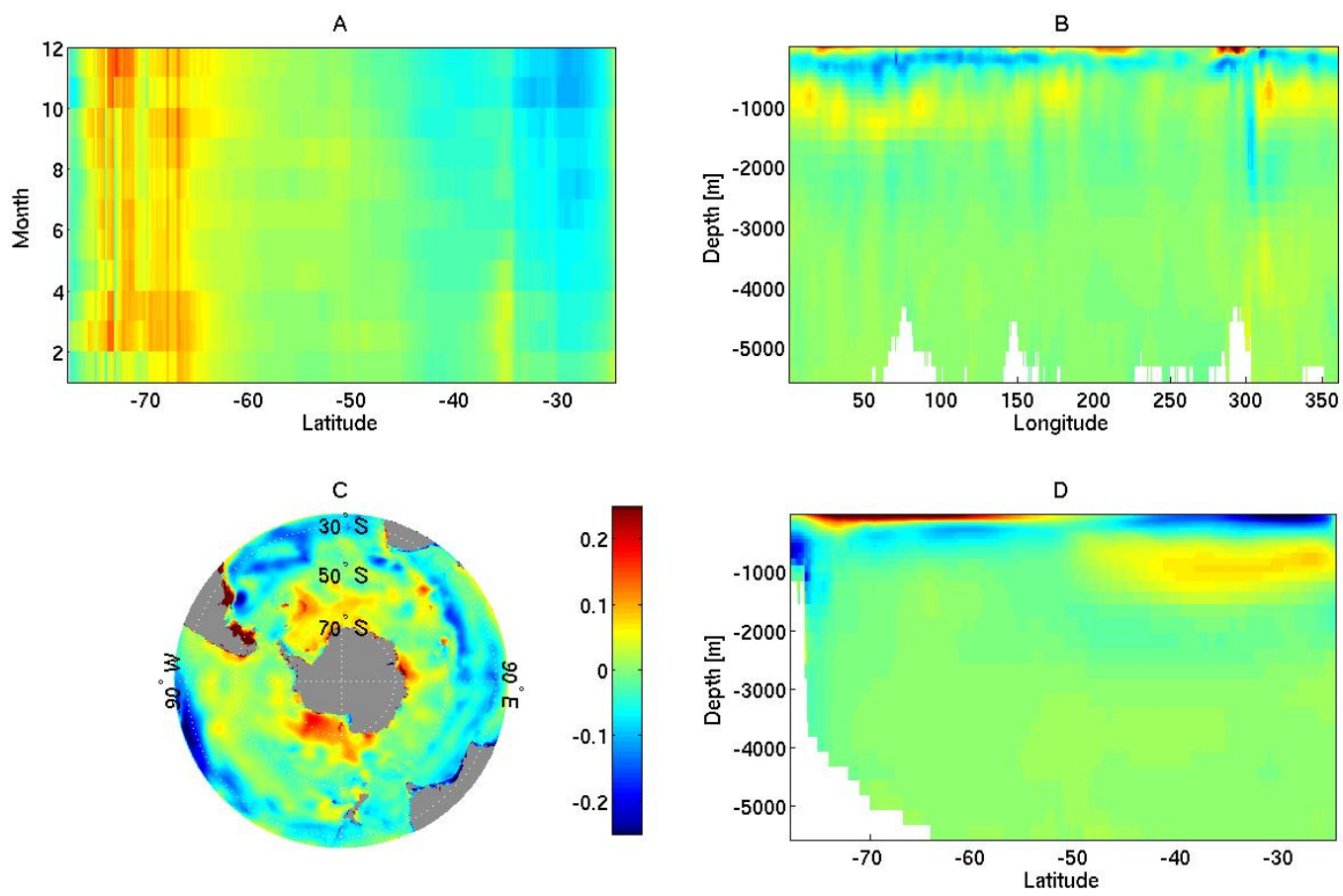

Figure 5-5: Model monthly salinity means minus climatology (positive values denote the model state is saltier than the climatology). A) Latitude vs. month (zonally and vertically averaged). B) Longitude vs. depth (latitudinally and temporally averaged). C) Longitude vs latitude (vertically and temporally averaged). D) Latitude vs. depth (longitudinally and temporally averaged).
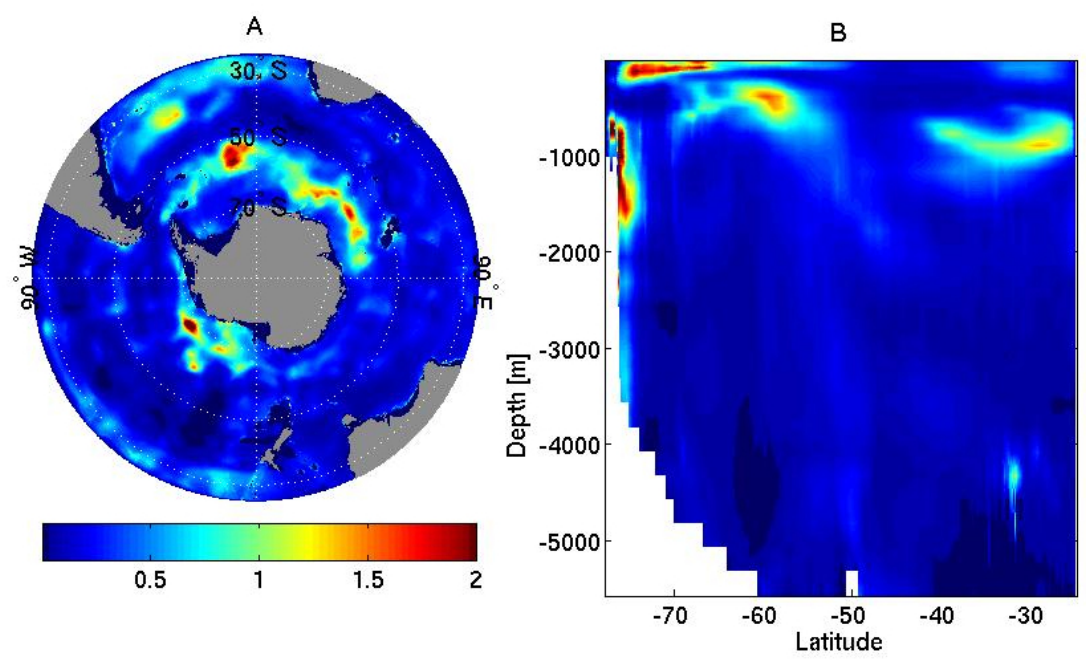

Figure 5-6: Cost associated with misfit shown in Figure (5-5). The cost equals the weighted (by uncertainty) misfit squared. Panels A and B are analogous to Panels C and D of Figure (5-5). 


\subsubsection{Altimetry}

The misfit of the model mean sea surface height to altimetry, and the cost associated with this misfit is shown in Figure (5-7). While some regions are consistent (for example, the eastern South Indian Ocean), there are still some some regions where much improvement is needed (east of the Drake Passage for example).
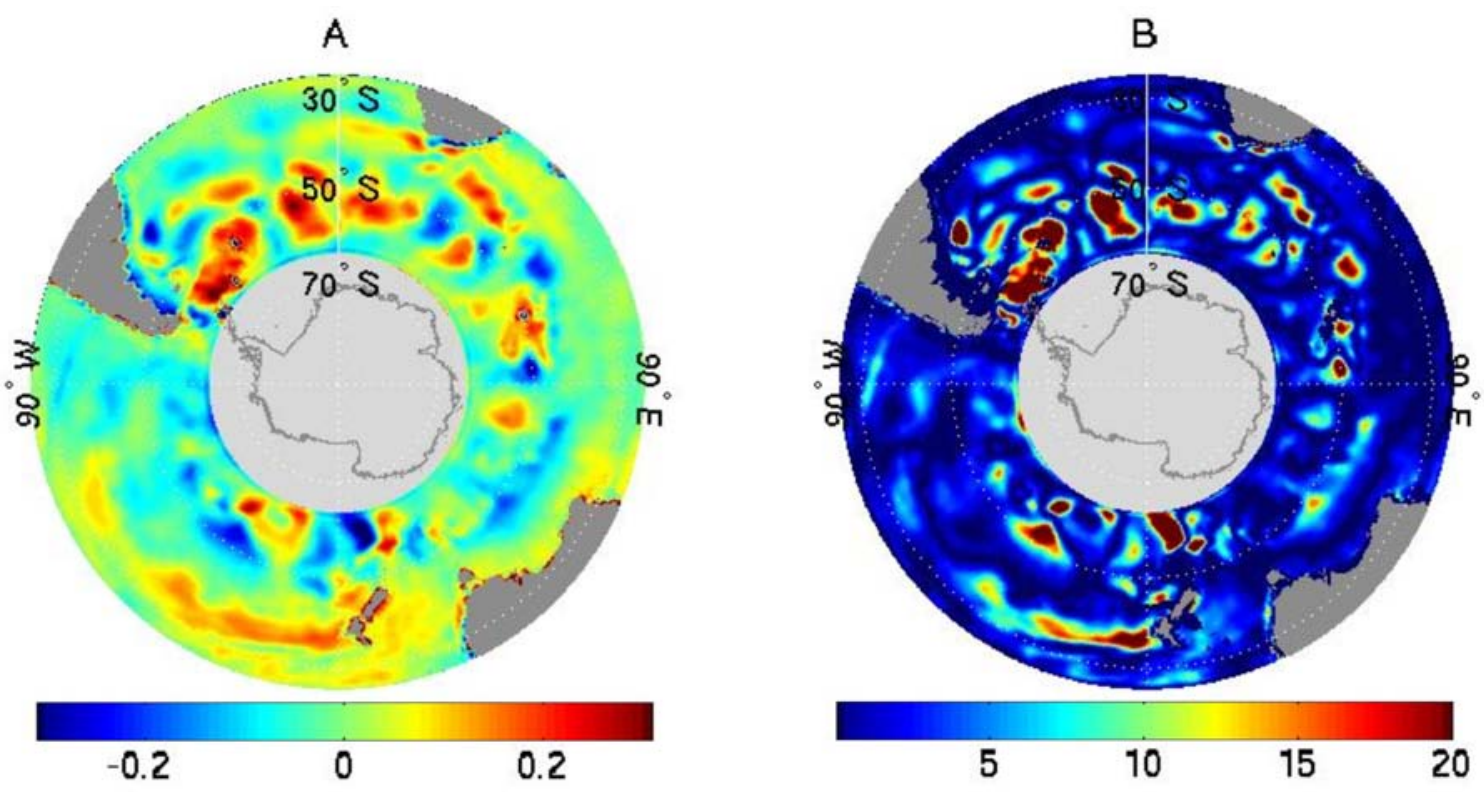

Figure 5-7: A) Mean model sea surface height misfit to altimetry in meters. B) Cost associated with this misfit.

Figure (5-8) shows the model sea surface height anomaly misfit to A) the Topex/Poseidon $(\mathrm{T} / \mathrm{P})$, and $\mathrm{B})$ the European Remote-Sensing (ERS) altimeter observed anomalies. The sea surface height anomaly is only available at locations along the altimeter orbital paths. Plots shown in Figure (5-8) are attained by binning and averaging the misfit over the full year of the state estimate. The misfit is then smoothed in order to be better visualized. The average misfit of each term over much of the region is order 0.1 meters, with the largest values along the path of the Antarctic Circumpolar Current. The acceptable misfit for sea surface height altimetric observations is also on the order of 0.1 meters. The cost, summed in time, is quite large however, with an average cost per term of about 16. Panel C in Figure (5-8) shows the summed cost contribution from sea surface height anomaly misfits. There are regions where the 
cost is quite high, for example in the South Pacific region of the Southern Ocean, especially around New Zealand. These high cost regions are, for the most part, scattered throughout the Southern Ocean
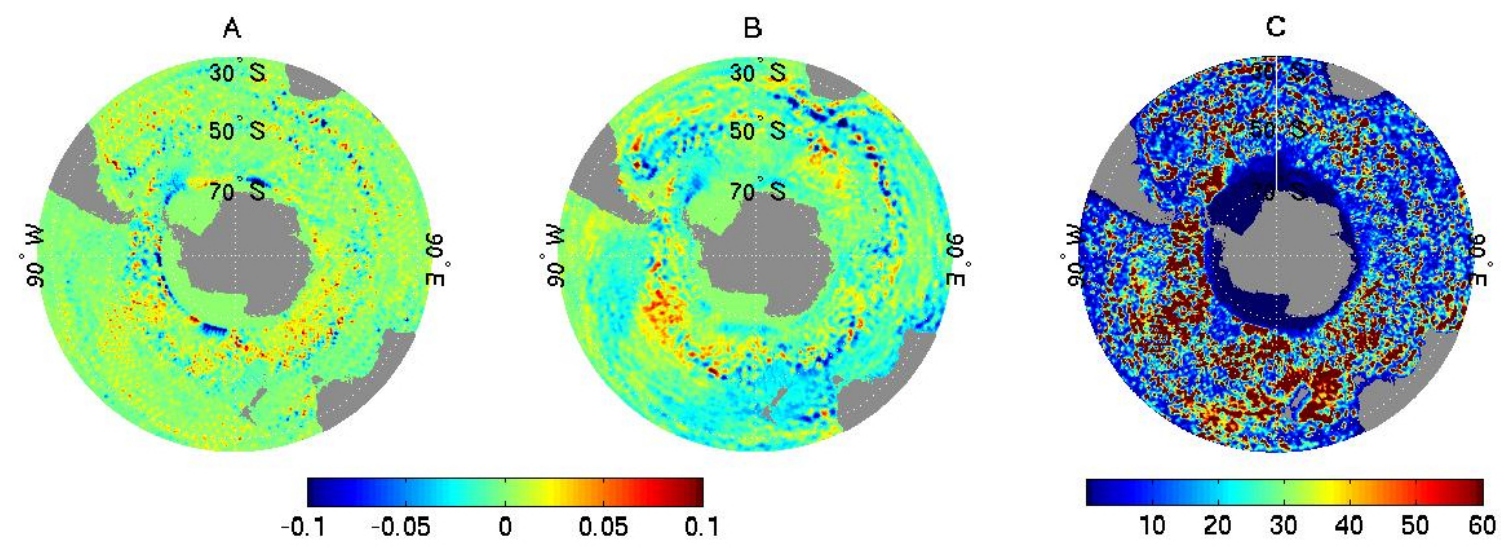

Figure 5-8: Sea surface height anomaly misfit of the model state from A) T/P and B) ERS observed anomalies. The misfit was binned and averaged over the state estimate duration, and then smoothed, in order to make the misfit more easily visualized. The colorbar (to the left) is misfit in meters. Panel $\mathrm{C}$ shows the combined cost (summed in time) associated with the misfit to the altimeters.

\subsection{Summary}

Remaining cost in the state estimate solution results from either an underestimate of the uncertainty in the data, an underestimate of the representation error in the model, or simply that more iterations are needed for the optimization to converge to an acceptable state. While uncertainty estimates for the model and the observations are imperfect, remaining misfits in the Southern Ocean state estimate primarily result from the need for more iterations to be carried out. Previous state estimates have taken on the order of 75 (Stammer et al., 2002; Ayoub, 2006) iterations to converge. Although the number of iterations needed for convergence is strongly dependent on how far the initial controls and the initial state deviated from acceptable values, 8 iterations is too few to expect a converged acceptable solution to be obtained. Figure (5-1) shows that the cost is still being considerable reduced, and there is no reason to expect this trend will not continue. 
A model ocean state has been brought closer to observations through the adjoint method. In select regions, this state is consistent with observations. Over the majority of the domain, however, the state is still unacceptable. Bringing the state into consistency with observations is possible through further iterations of the adjoint method. Carrying out these iterations is left for future work. 


\section{Chapter 6}

\section{The Southern Ocean General Circulation}

\subsection{Introduction}

The physical oceanographer's primary goals are to determine the general circulation of the ocean, and then to explain this circulation by uncovering which mechanisms dominate the dynamics. Much progress has been made towards both these goals, and a large part of this progress can be attributed to the tools of inverse calculations and numerical models. Combining inverse methods with high-resolution physically realistic numerical models, as was done in this work, now allows even greater light to be shed on the ocean circulation and the dynamics driving it. This final chapter covers the preliminary steps of analysis; that is, this chapter begins to describe the general circulation of the Southern Ocean. Determining the dominant dynamical processes driving this circulation is left for future work.

The state estimate used to describe the Southern Ocean's circulation is not fully consistent with observations. Chapter 5 described the "goodness" of the state estimate analyzed here. That "goodness" information can be use to gauge the accuracy of the inferences made in this chapter. Future work should determine a method for calculating accurate error bounds on the Southern Ocean circulation based on modelobservation misfit. For now, only the approximate circulation is presented and no 
attempt to gauge uncertainty is given.

The state estimate employs the Boussinesq approximation, and thus volume transport becomes the appropriate surrogate for mass transport. Section (6.2) examines the zonal volume transport. Though the meridional volume flux is higher order in the Southern Ocean, it is still of great interest in climate science because a large amount of water class transformation occurs in the Southern Ocean. The meridional transport is described in Section (6.3). To quantify this meridional transport of water masses, here characterized by their density signatures, it is useful to integrate zonally, and then calculate the meridional overturning streamfunction, $\psi$, where $\left(\bar{v}=\psi_{z}, \bar{w}=-\psi_{y}\right)$. As water bodies flow primarily along neutral density surfaces, the path of integration should follow these surfaces or spurious features may become present (Döös and Webb; 1994). For analyzing the pattern and approximate strength of the flow, using potential density referenced to 2000 decibars, $\sigma_{2}$, is a sufficient substitute for neutral density (Lee and Coward; 2003). A quantification of the meridional inter-ocean exchange along $\sigma_{2}$ surfaces is given in Section (6.3).

To gain insight into water masses flowing into and out of the Southern Ocean, it is beneficial to break up the overturning streamfunction into smaller regions. Three study regions are highlighted for analysis below. These regions, the South Atlantic Ocean, the South Indian Ocean, and the South Pacific Ocean, are denoted in Figure (6-1). This figure also shows the meridional cross-sections where the zonal transport is analyzed.

Throughout this chapter the reader must remember that this analysis is of preliminary results. Development of a state estimate is a substantial project and one should not wait for a converged solution to begin analysis. While the final converged solution will be different from the intermediate solution, the model grid will be the same. Developing software to analyze the state estimate can begin with the first iteration. Putting this software to use as early as possible is beneficial as well. For example, in the preliminary analysis presented in this chapter it is shown that, similar to many Southern Ocean models, the zonal transport is much larger than observed. This transport magnitude not only motivates several immediate questions, but also 


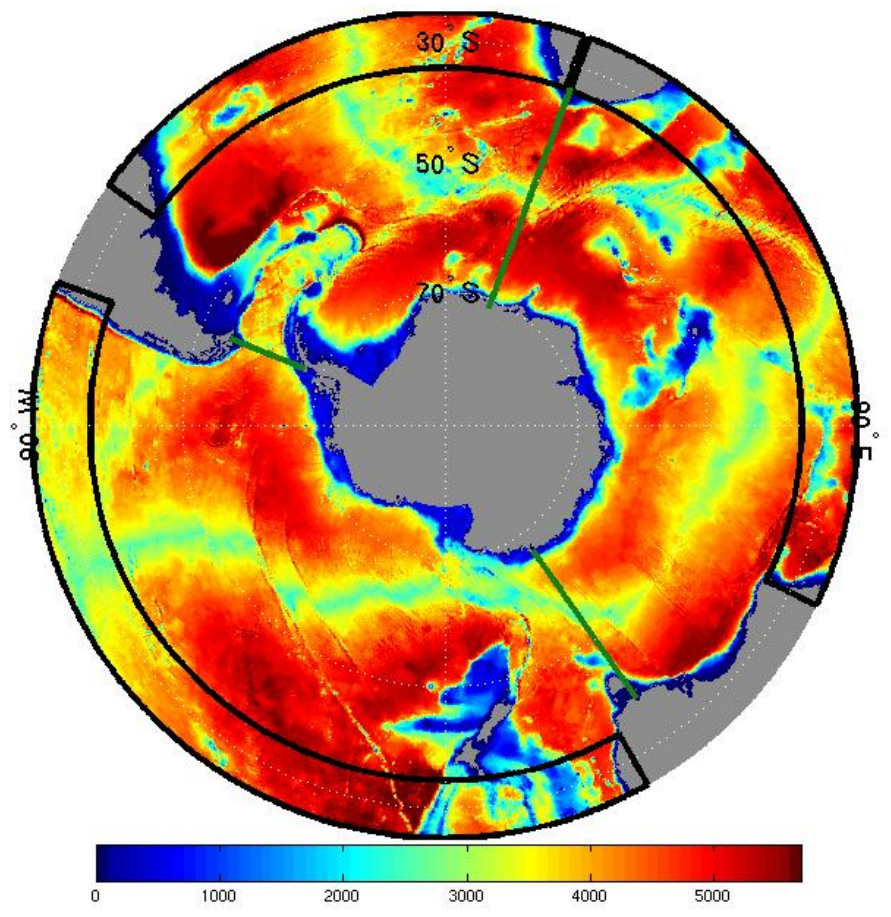

Figure 6-1: Bathymetry of the domain (colorbar is depth in meters). Regions where the zonal mean streamfunction was analyzed are outlined with thick black lines. Dark green lines show meridional sections where the zonal transport was analyzed.

provides a baseline to compare the converged solution too. If the converged solution has a zonal transport magnitude that is consistent with the observed estimate, determining what has driven this change will be insightful.

\subsection{Zonal Transport}

A standard diagnostic in numerical ocean models is the transport through the Drake Passage (the SR01 WOCE section). The observed annually averaged transport numerical models aim to reproduce is $134 \pm 27 \mathrm{~Sv}$ (Cunningham et al.; 2003). Consistency with this observed estimate is not readily achieved, in fact the bathymetry of many coarse resolution models is tuned in order to come closer to this value. Model estimates range from well under 100Sv, to well over 200Sv (Russell et al.; 2005). Most eddy permitting models come close, but are still well above, the preferred value 
(Olbers et al.; 2004). This state estimate is no exception, with an annual mean transport of $238 \mathrm{~Sv}$. The standard deviation of this transport time series, which excludes interannual variability, was $7 \mathrm{~Sv}$. This transport, along with the transport across meridional sections between Antarctica and Australia (the WOCE SR3 repeat section), and between Antarctica and South Africa, is plotted in Figure (6-2A). The SR3 section has a mean transport of $256 \mathrm{~Sv}$ with a standard deviation of $7 \mathrm{~Sv}$. The section south of Africa has a mean transport of $240 \mathrm{~Sv}$, also with a standard deviation of 7 Sv. From these transports an Indonesian throughway transport of approximately 17 $\mathrm{Sv}$ can be inferred. (One may claim that $16 \mathrm{~Sv}$ must pass through the Indonesian throughway and $2 \mathrm{~Sv}$ must pass through the Arctic Ocean, but the magnitude of the uncertainty in the zonal transport makes this claim a bit far-fetched).

The temporal correlation of the total transport between slices, as shown in Figure (6-2 A), is remarkable. The transport magnitude signal travels much faster than the fluid moves itself. It is possible that the fastest fluid parcels moving on a direct path may be able to make it from one section to the next on the order of 1 month. The plot shows, however, not even a 1 week lag between sections. It is impossible that Rossby waves could be transmitting this signal, since they propagate westward and are therefore slowed by advection from the eastward moving Antarctic Circumpolar Current. A more likely cause of this highly temporally correlated transport is atmospheric forcing, since atmospheric signals, and the barotropic oceanic responses to them, are capable of moving this quickly. Coherent patterns of circumpolar atmospheric forcing have been found, and fluctuations in this forcing have been shown to correlate with sub-surface pressure gauges around Antarctica (Hughes et al.; 2003). In further support of the hypothesis that barotropic responses to atmospheric forcing is responsible for this coherent circumpolar transport variability, several studies have shown that changes in the zonal wind stress in a model result in a change in the zonal transport (Hallberg and Gnanadesikan; 2005; Webb and de Cuevas; 2006). Hughes et al. (1999) found a strong correlation between the zonal transport variability in the FRAM model and the zonally averaged wind at $65^{\circ} \mathrm{S}$. They found an even stronger correlation with the transport variability and the wind stress curl poleward of $65^{\circ} \mathrm{S}$, 

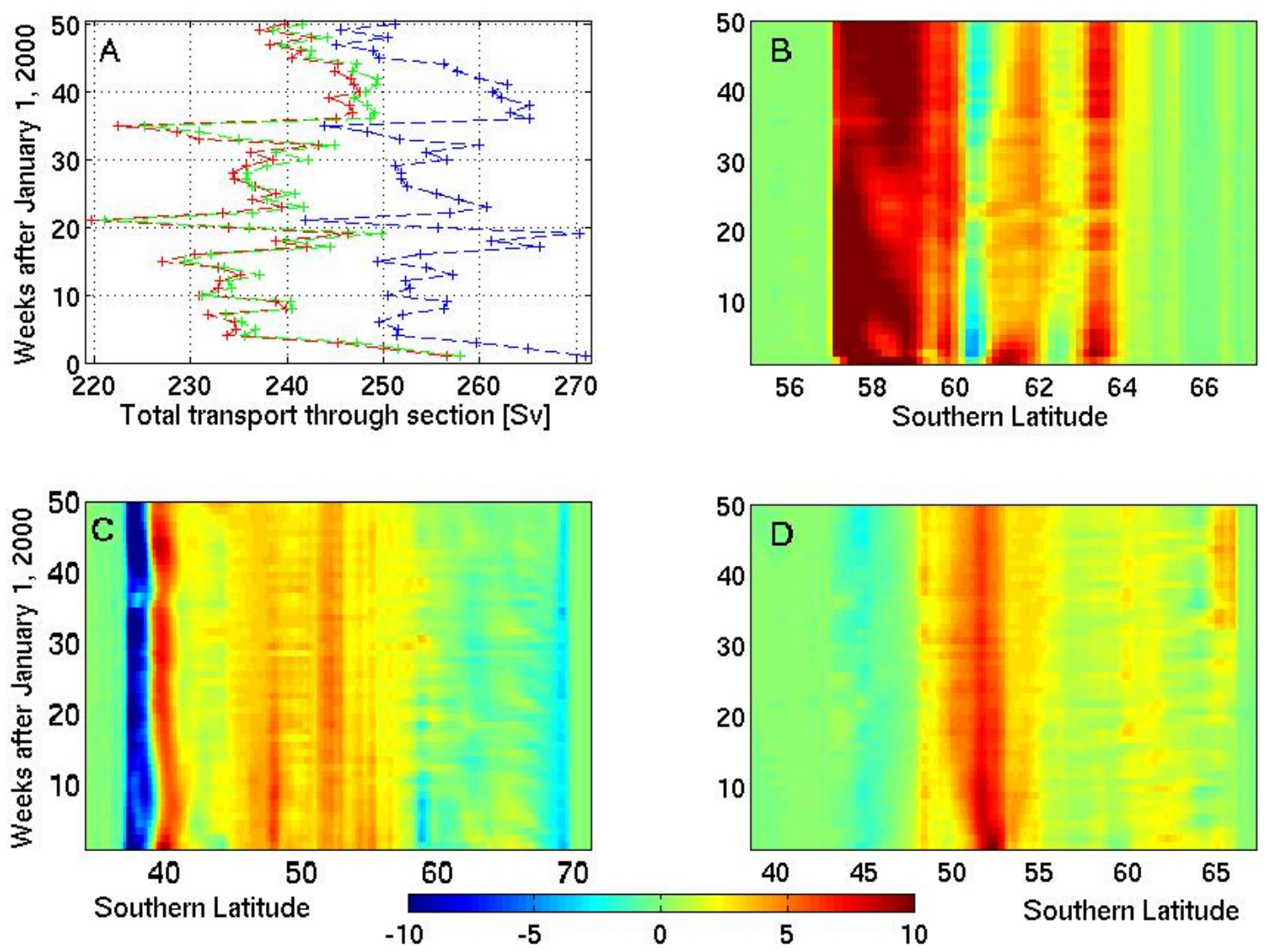

Figure 6-2: Panel A is the total zonal transport between South America and Antarctica (red), Africa and Antarctica (green), and Australia and Antarctica (blue) as a function of time. Panel B, C, and D shows the vertically integrated transport through these respective sections. The colorbar denotes the transport in Sverdrups. See the green meridional lines in Figure (6-1) for the exact location of these sections.

which suggests the strength of the Subpolar Region circulation is significant in setting the zonal Antarctic Circumpolar Current transport magnitude. An initial test of this wind driven zonal transport hypothesis is given in Figure (6-3). This figure plots the mean meridional and zonal wind speeds along the section between Africa and Antarctica. The mean wind speeds and the zonal transport across this section are not significantly correlated; the correlation coefficient for both wind speeds to transport is approximately -0.1. Clearly the relationship between the atmospheric forcing and the zonal transport variability is complex. Future work should investigate this relationship, and try to determine why there is an insignificant lag along these three meridional sections that are separated by such a great distance. 


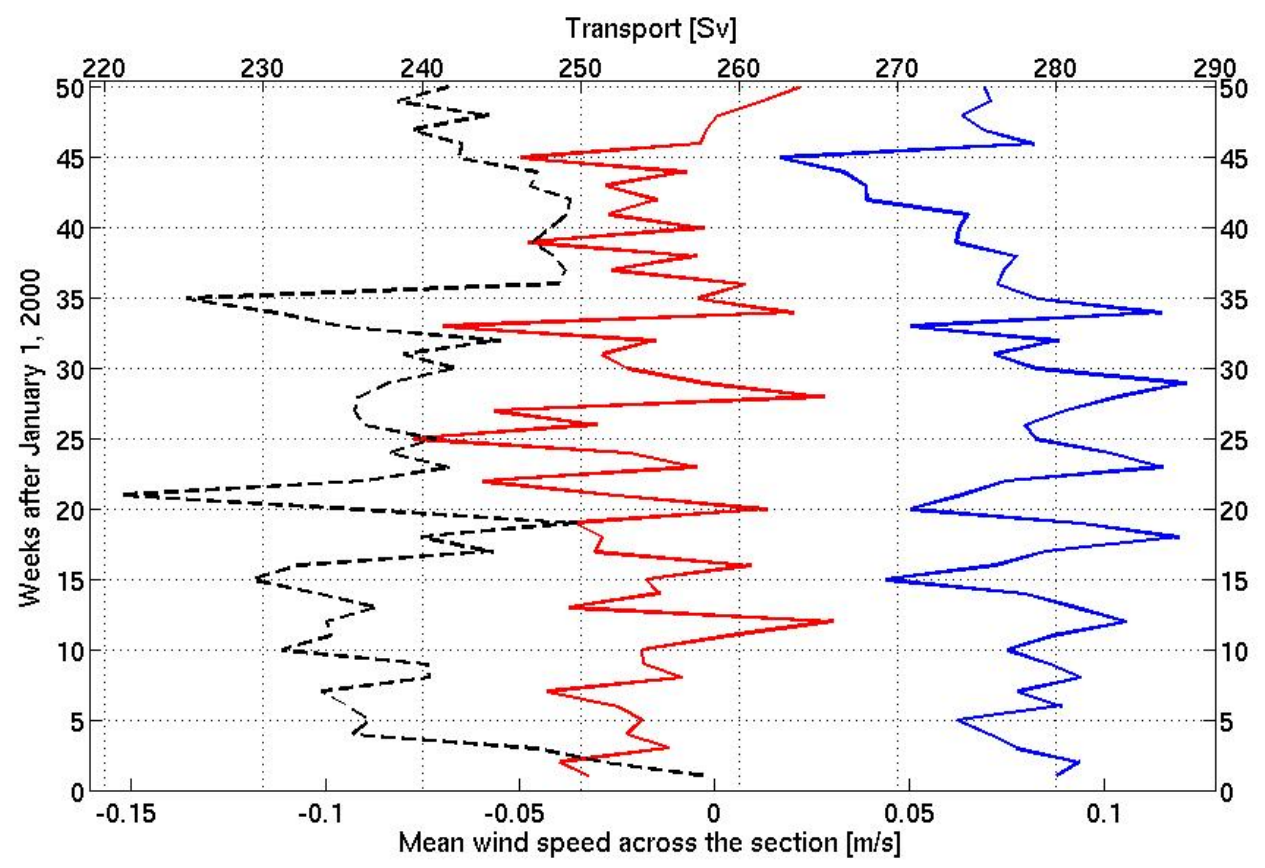

Figure 6-3: The dashed black line is the total transport between Africa and Antarctica in Sverdrups as a function of time. The blue and red lines are the mean zonal and meridional wind speeds across this section respectively. These are sampled at the same frequency as the transport.

Figure (6-2 B,C,\&D) show the vertically integrated transport along the sections from Antarctica to South America, Africa, and Australia respectively. This shows that, for the most part, the large transport is actually achieved in narrow fronts. Where the influence of topography is less significant, the Subantarctic and Polar Fronts are quite distinguishable. In some cases the so-called Southern Front, which is located poleward of the Polar Front, is also distinguishable. Though topography is influential in the three sections shown, the two frontal regions are still distinguishable. Also notable in the sections are the (continental) boundary currents, which often oppose the Antarctic Circumpolar Current transport. The most notable of these currents is the Agulhas overshoot, which can be seen in Figure (6-2 C) to have a transport on the same order as what is found along the Subantarctic front. These two strong opposing flows make it easy to understand why this is one of the most 
turbulent and eddy rich regions of the World Oceans.

The large discrepancy between the observational transport estimate and the state estimate's transport value may be due to the fact that the state estimate is not converged. The results analyzed are preliminary; as was shown in Chapter 5, there are large regions over the Antarctic Circumpolar Current that are inconsistent with the observed mean sea surface height. It is almost certain that the mean Antarctic Circumpolar Current transport will vary as the mean sea surface height changes. There are other possible reasons for this inconsistency, for example an improper parameterization of small scale ocean dynamics or an inappropriate representation of bottom topography could contribute to the discrepency. The ECCO $1^{o}$ global state estimate used to initialize this model does give a zonal Antarctic Circumpolar Transport consistent with observations. Possible reasons for this difference in the current high-resolution model are discussed in Section (6.4).

\subsection{Meridional Transports}

The Southern Ocean ventilates large regions of the World Oceans, with its signatures found even in the North Atlantic intermediate waters (Marsh et al.; 2000). This role in ventilation stresses the importance of the Southern Ocean to the global climate. To quantify and understand the Southern Ocean's role in climate it is necessary to determine its meridional overturning circulation. This section describes this Southern Ocean circulation.

It is natural for scientists to view the oceans in depth coordinates, indeed this is how humans (and the MITgcm) view the world. The proper vertical reference frame for ocean dynamics, however, is along lines of neutral density. A procedure to calculate the meridional transport (or any physical processes) in density space, and then portray the result in depth space, is extremely useful. A framework to do this was derived for temporally constant flows by McIntosh and McDougall (1996). Lee and Coward (2003) appended a temporal dependence to this derivation. A more complete

derivation of the the meridional overturning streamfunction than that published in 
these two works is given in Appendix A. Below the result is given, and the physical significance of the terms are noted.

The goal is to find the zonal integral of the time mean flux of a property, $C$, between two isopycnal layers, $\rho_{1}$ and $\rho_{2}$, of depth $z_{1}$ and $z_{2}$ :

$$
\int_{x_{W}}^{x_{E}} \frac{1}{\tau} \int_{0}^{\tau} \int_{z_{2}\left(x, y, \rho_{2}, t\right)}^{z_{1}\left(x, y, \rho_{1}, t\right)} v C d z d t d x
$$

where $v$ is the meridional velocity, $\tau$ is the duration of the analysis period, and $C$ is any fluid property in question (e.g. heat content). To determine the volume transport between the isopycnals one would set $C=1$.

To gain insight into the processes at work in this transport, variables are separated into mean components and perturbations from the means: $v=\bar{v}+v^{\prime}=[\bar{v}]+\bar{v}^{*}+v^{\prime}$. Here an overbar denotes a time mean, a square bracket denotes a zonal mean, a prime denotes a deviation from the time mean, and an asterisk denotes a deviation from the zonal mean. This is also done for the isopycnal depth such that $z_{n}\left(x, y, \rho_{n}, t\right)=$ $\overline{z_{n}}+z_{n}^{\prime}=\left[\overline{z_{n}}\right]+{\overline{z_{n}}}^{*}+z_{n}^{\prime}$. In the derivation the assumption is made that perturbation values are higher order than the mean. That is, while barred and bracketed variables are considered $\mathrm{O}(1)$, primed and starred variables are considered $\mathrm{O}(\epsilon)$ where $\epsilon<<1$. The calculation is carried out to second order; all terms of $\mathrm{O}\left(\epsilon^{3}\right)$ are neglected. The validity of this approximation is discussed below.

The analysis given in this chapter focuses on the volume transport, and thus $C$ is set to $1 . h$ is defined to be the isopycnal thickness, and $L$ is defined to be length of zonal integration $\left(L \equiv \int_{x_{W}}^{x_{E}} d x\right)$. Keeping $h$ finite, but taking the limit where $\rho_{1}\left(z_{1}\right)$ approaches $\rho_{2}\left(z_{2}\right)$, such that $z_{1} \approx z_{2} \approx z$, one finds (see Appendix A)

$$
\begin{aligned}
{[\bar{h}]^{-1} \int_{x_{W}}^{x_{E}} \frac{1}{\tau} \int_{0}^{\tau} \int_{z_{2}}^{z_{1}} v d z d t d x=} & L[\overline{h v}] /[\bar{h}] \\
= & L[\bar{v}] \\
& -L\left[\bar{v}^{*} \bar{\rho}^{*}\right]_{z} /\left[\overline{\rho_{z}}\right] \\
& -L\left[\overline{v^{\prime} \rho^{\prime}} / \overline{\rho_{z}}-(1 / 2)(\bar{v})_{z} \overline{\rho^{\prime 2}} / \overline{\rho_{z}}\right]_{z} .
\end{aligned}
$$


This equation gives the zonally integrated and time mean meridional volume transport in an isopycnal layer. In the continuum limit where the isopycnal layer thickness approaches zero, Equation (6.3) gives the zonally integrated and time mean transport along isopycnals. The only assumption made in its derivation is that perturbations from the mean are small. When the vertical density gradient approaches zero this assumption is violated. As is shown in Appendix A, when $\rho_{z}$ becomes small, perturbations in the layer thickness can be of very large amplitude. It is to be expected that the analysis below will be robust over much of the ocean. There are two regions, however, where $\partial \rho / \partial z$ is very small and the formulation may break down. These two regions are near the Antarctic Continent (specifically in the Ross and Weddell Seas), and in the surface mixed layer. In these regions caution must be taken in evaluating the meridional transport with this formulation.

Integrating the continuity equation $\left(u_{x}+v_{y}+w_{z}=0\right)$ zonally, either between continents where $u$ must be zero, or around full latitude circles where $u$ is periodic, gives $L[v]_{y}+L[w]_{z}=0$. One may therefore define a streamfunction $\left(\psi_{z},-\psi_{y}\right)=$ $(L[v], L[w])$, which ensures continuity. This is the streamfunction for the Eulerian mean circulation. The Eulerian streamfunction, which is attained by vertical integration of term (6.3a), does not take into account isopycnal meanders from latitude circles. Neglecting these meanders causes spurious circulation features(Döös and Webb; 1994). The transformed Eulerian mean streamfunction, also known as the residual mean streamfunction, is defined as $\psi_{z}^{\dagger}=L[v h] /[h]$. This is the zonally integrated volume weighted isopycnal transport of Equation (6.2). It is desirable to analyze, and work with the residual mean circulation, as buoyancy and other water properties (e.g. CFCs) are advected by this velocity. In the residual mean circulation framework, no "eddy" terms show up explicitly in the buoyancy equation; only diabatic processes force property transport across $\psi^{\dagger}$ streamlines. Equation (6.2) shows that the eddy terms, (6.3b) and (6.3c), are subtracted from the Eulerian circulation, (6.3a), leaving the residual mean circulation.

Physical interpretation of the "eddy" terms is relatively straightforward. The 
residual mean transport, Equation (6.2), is the mean volume flux within an isopycnal layer. The layer thicknesses vary in space and time. When integrating zonally, the volume flux resulting from these thickness perturbations is accounted for by the eddy terms. The Eulerian mean transport, $L[\bar{v}]$, is the volume transport derived by integrating around latitudinal circles; it does not account for layer thickness variations. The thermal wind relation shows that, when integrated zonally, time mean layer stretching in the presence of a background velocity shear results in a higher order meridional transport (see Appendix A). A significant mean transport can result, however, from velocity perturbations being correlated with the time mean layer thickness perturbations. Term (6.3b), which is often referred to as the "standing eddy" term (suggesting standing eddies are responsible for this isopynal stretching and velocity perturbation), accounts for this transport. Transient features of the flow, for example from convective events or frontal instabilities, are accounted for in the so-called "transient eddy" terms. These terms, (6.3c), show that isopycnal layer stretching correlated with time varying velocity perturbations, or in the presence of a background velocity shear, may cause a significant transport within an isopycnal layer.

A standing and transient eddy streamfunction may be defined as

$$
\begin{gathered}
\psi_{z}^{s}=-L\left[\bar{v}^{*} \bar{\rho}^{*}\right]_{z} /\left[\overline{\rho_{z}}\right] \\
\psi_{z}^{t}=L\left[-\overline{v^{\prime} \rho^{\prime}} / \overline{\rho_{z}}+(1 / 2)(\bar{v})_{z} \overline{\rho^{\prime 2}} / \overline{\rho_{z}}\right]_{z} .
\end{gathered}
$$

The residual stream function becomes $\psi_{z}^{\dagger}=\psi_{z}+\psi_{z}^{s}+\psi_{z}^{t}$. Vertically integrating from the bottom to depth $z$ allows calculation of the residual mean streamfunction from the meridional velocity and the density: 


$$
\begin{aligned}
\psi^{\dagger} & =\int_{z_{b}}^{z} L[\overline{v h}] /[\bar{h}] d z \\
& =\int_{z_{b}}^{z}[\bar{v}] d z-L\left[\bar{v}^{*} \bar{\rho}^{*}\right] /\left[\overline{\rho_{z}}\right]-L\left[\overline{v^{\prime} \rho^{\prime}} / \overline{\rho_{z}}\right]+L\left[(\bar{v})_{z} \overline{\rho^{\prime 2}} / 2 \overline{\rho_{z}}\right]
\end{aligned}
$$

Bottom contributions from standing and transient eddies vanish in the above equation because the no-slip boundary condition dictates that the mean and perturbation meridional velocity both vanish identically at the sea floor. The residual mean transport is determined to within a constant (which was set to zero at the bottom), and it is important to remember that actual transports are proportional to the difference between values at different depths.

The residual mean circulation is plotted in Figure (6-4). As expected, the values are questionably large near Antarctica and in the surface mixed layer; these are regions where the assumptions made above may break down. Nevertheless, the streamfunction plotted gives a good qualitative look at the Southern Ocean meridional overturning. Upper Deep Water enters the Southern Ocean approximately between 1,500 meters and 4,000 meters. This water upwells between $\sim 27^{\circ} \mathrm{S}$ and $\sim 35^{\circ} \mathrm{S}$. Convergence at the Subtropical front causes subduction at $\sim 37^{\circ} \mathrm{S}$. This thermocline water joins with the upwelled deep water in a strong equatorward flow. A significant portion of the upwelled upper deep waters never enter the Antarctic Circumpolar Current because the strong fronts of the Antarctic Circumpolar Current act as a barrier to their transport. This barrier effect is apparent from the weak meridional flow poleward of $40^{\circ} \mathrm{S}$ at depths between $500 \mathrm{~m}$ and $3500 \mathrm{~m}$. South of these fronts, and at mid-depths, another large circulation is found. Water sinks near the Antarctic coast and then recirculates, upwelling just south of the polar front. At depths above 500 meters, and below 3500, meters some of this water escapes this near-continent recirculation. At depths below 4,000 meters and north of the Drake Passage latitudes, a relatively strong outflow of bottom water is found. This outflow is a mix of waters formed near Antarctica and of poleward flowing Lower Circumpolar Deep Wa- 


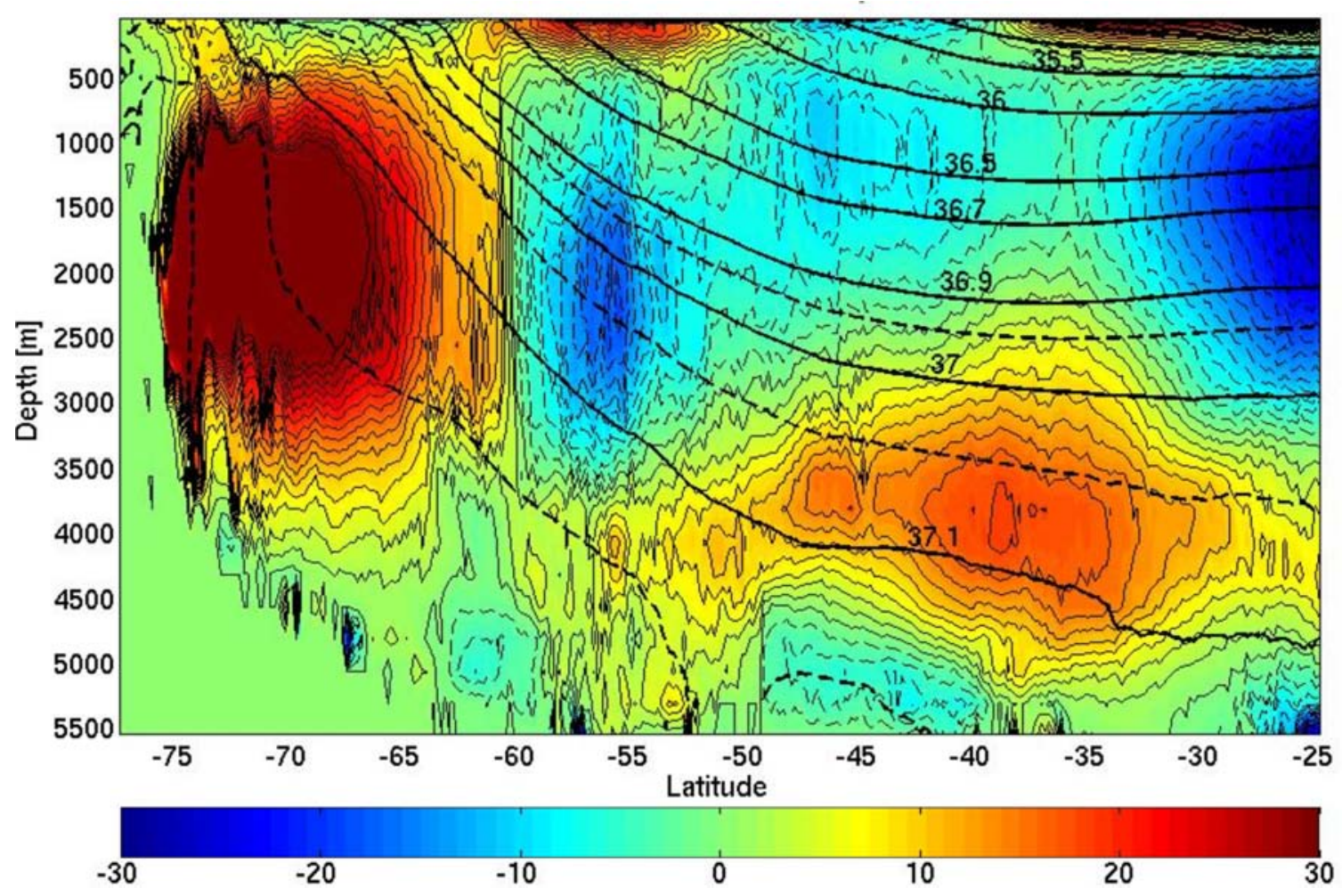

Figure 6-4: Residual mean streamfunction, $\psi^{\dagger}$, in Sverdrups (Sv) for the state estimate domain. The contour interval is $2 \mathrm{~Sv}$. Positive values denote counter-clockwise circulations, and negative values, which have dashed contours, denote clockwise circulations. Temporal and zonal mean $\sigma_{2}$ (potential density referenced to $2000 \mathrm{db}$ ) contours are overlayed. Note the $\sigma_{2}$ contour intervals change from $0.5 \mathrm{~kg} / \mathrm{m}^{3}$ to 0.1 $\mathrm{kg} / \mathrm{m}^{3}$ at $\sigma_{2}=36.5 \mathrm{~kg} / \mathrm{m}^{3}$. Dashed contours represent $\sigma_{2}=37.05 \& 37.15 \mathrm{~kg} / \mathrm{m}^{3}$.

ter. How much each of these two sources contributes to this volume of equatorward flowing bottom water has important climate implications, as the age since ventilation for the two water masses is very different. A significant feature of the residual mean streamfunction in Figure (6-4) is a substantial diapycnal flow. This is also found when the streamfunction is calculated along isopycnals. Discussion of this result is deferred to Section 6.4.

It should be noted that the residual streamfunction plotted is in good agreement with others derived for similar models, e.g. FRAM (Döös and Webb; 1994) and OCCAM (Lee and Coward; 2003). One difference is the state estimate finds a stronger overturning at depth than that of FRAM. Also FRAM and OCCAM both find a negligible overturning poleward of $65^{\circ} \mathrm{S}$. In contrast to this, the inverse calculation of 
Sloyan and Rintoul (2001b) finds a near-continent overturning on the order of $50 \mathrm{~Sv}$ which is of the same magnitude found in the residual streamfunction calculation in this work. The amplitude of this streamfunction in this region, however, is questionable (as explained above).

Many factors account for discrepancies between numerical model solutions, ranging from choice and implementation of boundary conditions to spatial resolution. The rest of this section will focus on the difference in the circulation inferred from the Southern Ocean state estimate produced, and that inferred directly from observations. For purposes of quantitative comparison, however, the formulation above, with its assumptions of small isopycnal perturbations from the mean, is inferior to a calculation of the streamfunction in isopycnal space. Converting the model state from length coordinates to isopycnal coordinates is computationally intensive. The problem is made more tractable, however, if the transformation is made only on the time-mean state. It is possible to determine if this state is a good representation of the full time variable transport, because the formulation of the residual mean circulation given above, with its separation of the temporal mean and anomalous state, reveals the significance of the transient circulation to the mean transport.

The transport resulting from the time variable circulation is shown in Figure (6-5). To determine the contribution of transients, the model state was sampled at 7 day intervals. This is a sufficient sampling interval as altimeter data indicates an e-folding scale of 34 days for temporal variability in the Southern Ocean (Gille; 1995). Transient features drive a deep and narrow subduction cell around $68^{\circ} \mathrm{S}$. The time variable flows also play a role in the Antarctic Circumpolar Current, forming several small recirculations at frontal latitudes. The transient circulation appears to be significant near the surface, which is likely due to temporal shifts in the wind stress location and strength. The transient component of the meridional overturning circulation is, however, higher order. Except in locations where the time mean circulation goes to zero, the ratio of the magnitude of the transient component to the time mean component is order 0.1 (see Figure (6-6)). The results that follow focus on the time mean streamfunction calculated in isopycnal space. Neglecting the transient circulation is 


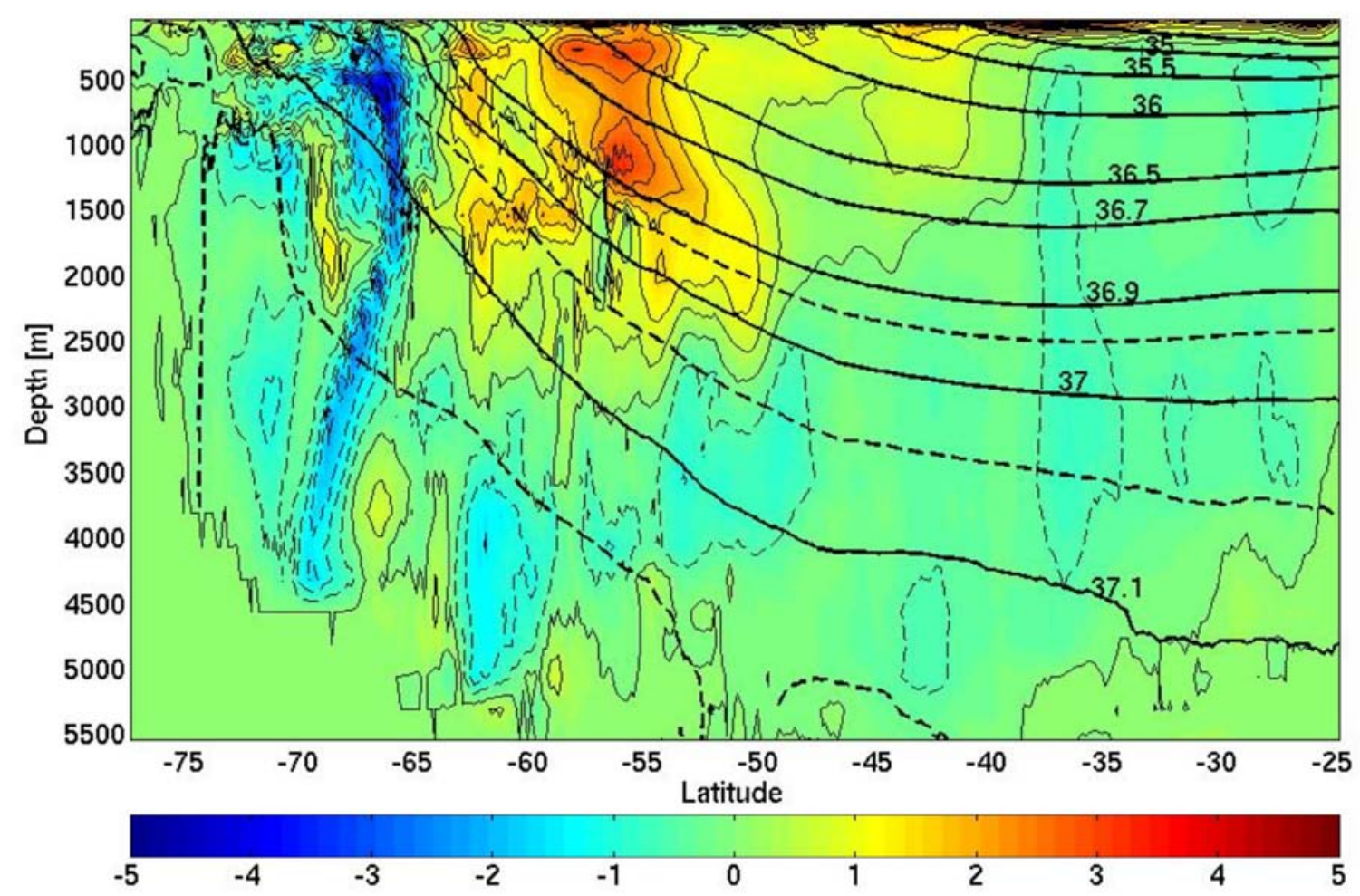

Figure 6-5: Contribution to the residual mean streamfunction from the time variable circulation. The contour interval is 0.5 Sverdrups. $\sigma_{2}$ contours are overlayed.

justified by Figure (6-6), but there is associated error in this omission that should not be overlooked.

The time mean meridional transport calculated in isopycnal coordinates (potential density referenced to 2000 decibars is the vertical coordinate) is shown in Figure (6-7). While Figure (6-4) may be more readily interpreted, Figure (6-7) allows the transport to be determined more accurately. This is especially true near Antarctica, where not only is the streamfunction magnitude more reasonable, but it can now also be seen that two circulation cells exist. Both circulations have magnitudes on the order of 10 Sv. Wind drives the surface waters towards the Antarctic continent where they enter boundary currents and eventually subduct. The subducted waters flow northward rising to the surface again at the Polar Front. This upper cell circulation is of a counter-clockwise orientation. Some of the subducted waters reach to greater depths where they enter a separate deep cell. This deeper cell is characterized by a clockwise circulation. 


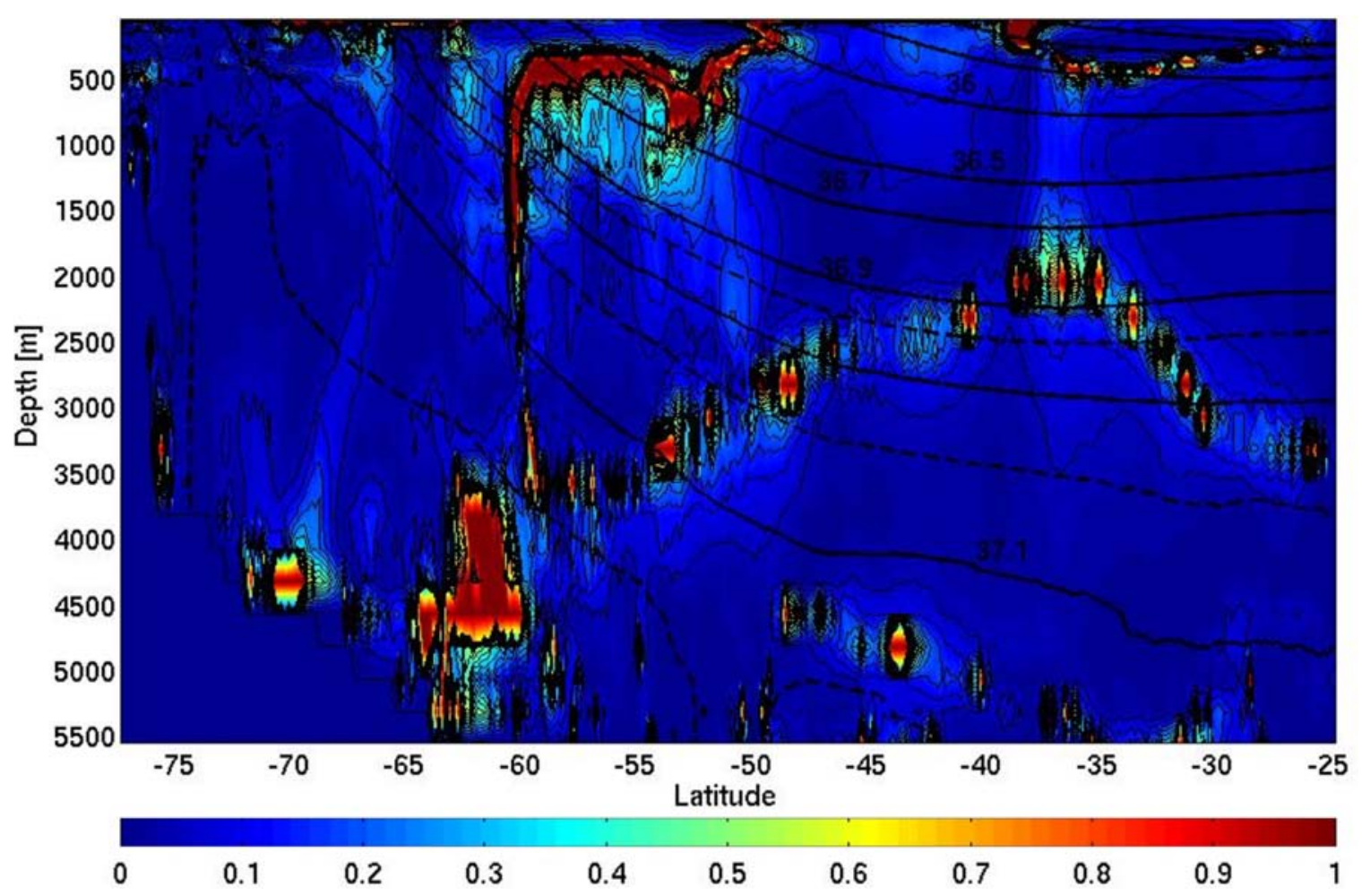

Figure 6-6: Ratio of the time variable streamfunction magnitude to the time mean streamfunction magnitude. $\sigma_{2}$ contours are overlayed.

Equatorward of the Polar Front another large wind driven circulation exists. This clockwise circulation cell, which spans the latitudes from $55^{\circ} \mathrm{S}$ to $40^{\circ} \mathrm{S}$, drives surface waters equatorward. The cell reaches its maximum transport value of about $40 \mathrm{~Sv}$ around $48^{\circ} \mathrm{S}$. The water continues north, gaining buoyancy as it goes, until dramatically weakening upon reaching a weaker southward flow at the Subtropical Front at $\sim 40^{\circ} \mathrm{S}$. The southward flow interacting with this cell is actually two separate circulations, one near the surface and one at depth. The deep inflow, with density $34.0<\sigma_{2}<36.3$, has a magnitude of about $25 \mathrm{~Sv}$. A portion of the denser inflow waters lose buoyancy and return equatorward before ever crossing into the Antarctic Circumpolar Current latitudes. This implies substantial mixing is occurring around the Subtropical Front. Much of the lighter poleward inflow penetrates far into the Southern Ocean before being upwelled at the Polar Front. Hallberg and Gnanadesikan (2005) have experimented with models of various resolutions and found that this inflow was only able to cross the Subantarctic Front and penetrate to the Polar Front 


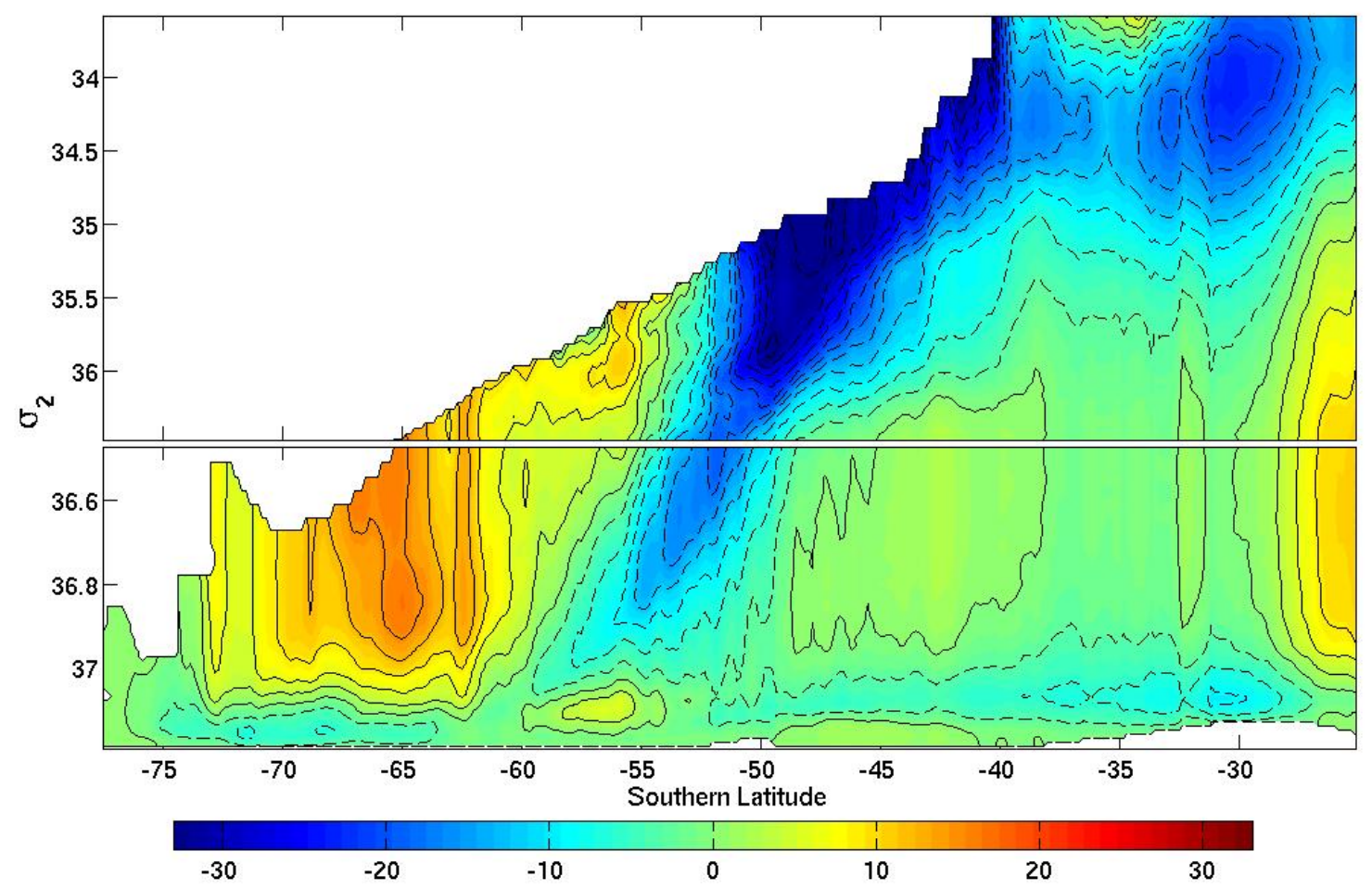

Figure 6-7: Temporal and zonal mean streamfunction calculated in isopycnal space for the state estimate domain. The contour interval is $2 \mathrm{~Sv}$. Note the change of scale at $\sigma_{2}=36.5 \mathrm{~kg} / \mathrm{m}^{3}$.

at eddy permitting resolutions, suggesting eddies are the means for which waters may cross the Southern Ocean's strong fronts.

The description of the meridional circulation given above by no means implies a strictly two-dimensional flow in the Southern Ocean. On the contrary, the meridional overturning depicted in Figure (6-4) and Figure (6-7) occurs in the background of the much larger zonal flow described in Section (6.2). Much of this overturning circulation can in fact be attributed to meridional and vertical meanders of the predominantly zonal streamlines. ${ }^{1}$ A net meridional transport is achieved, however, and its impact on the global ocean circulation is far from trivial.

Many studies have attempted to use observations to quantify the flux of water (and its properties) into, and out of, the Southern Ocean. Figures (6-8), (6-9), and (6-10)

\footnotetext{
${ }^{1}$ In general, the Antarctic Circumpolar Current exhibits a gradual southward shift across the Atlantic, Indian, and Pacific basins, and a strong northward shift (the Falkland Current) along the eastern coast of South America.
} 


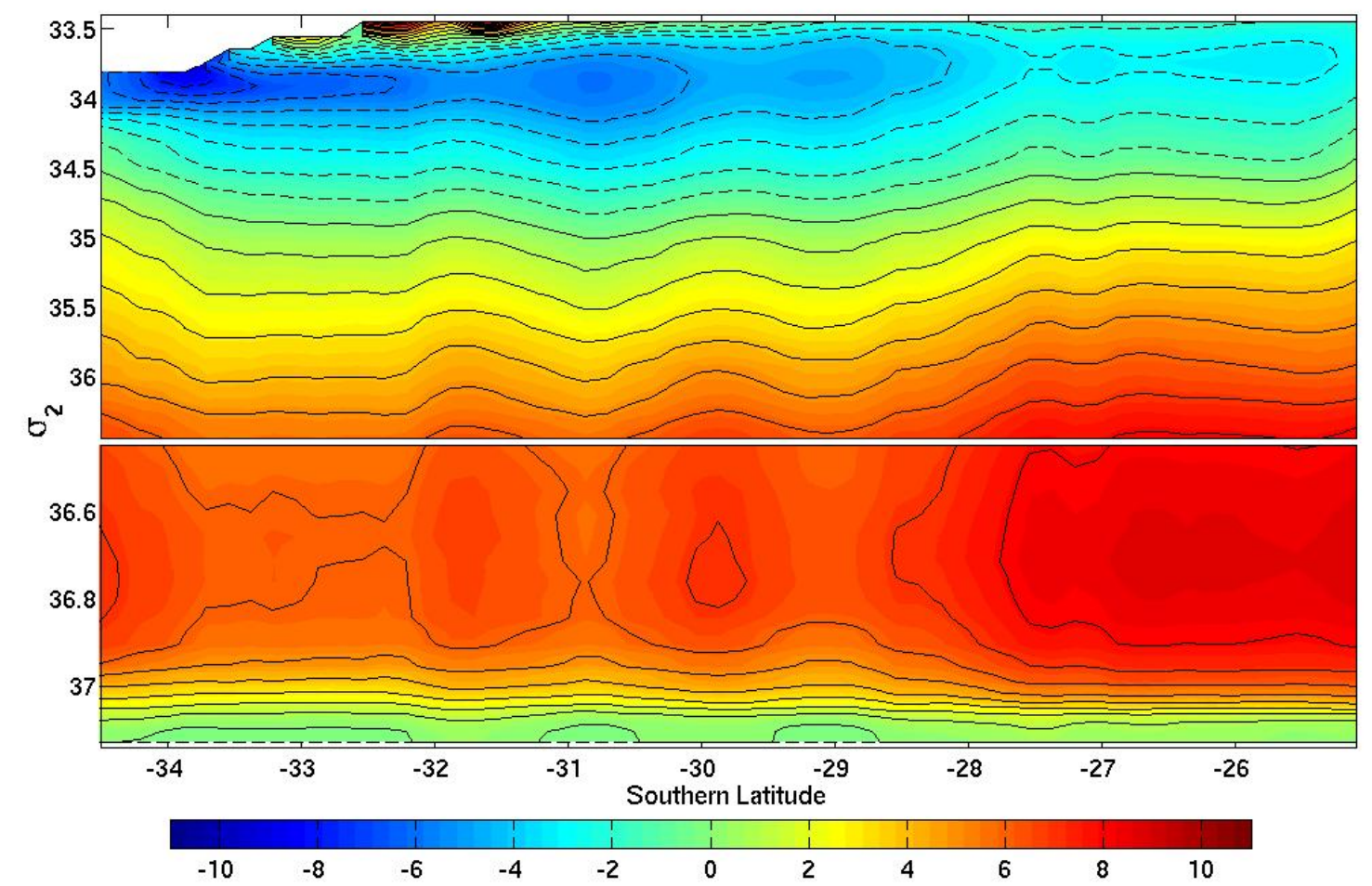

Figure 6-8: Temporal and zonal mean streamfunction calculated in isopycnal space for the South Atlantic Ocean. The contour interval is $1 \mathrm{~Sv}$.

show the meridional overturning streamfunction in the South Atlantic, South Indian, and South Pacific Oceans. The South Atlantic overturning streamfunction (Figure (6-8)) shows about $12 \mathrm{~Sv}$ of North Atlantic Deep Water entering the domain from the South Atlantic. South of $27^{\circ} \mathrm{S}$ this poleward flow mixes with denser deep waters, with some of it entraining into the dense water outflow. Poleward of approximately $30^{\circ} \mathrm{S}$ the North Atlantic Deep Water inflow mixes with less dense surface waters, entraining them and slowly increasing its transport to $18 \mathrm{~Sv}$ by $34^{\circ} \mathrm{S}$. Mass is balanced by outflows into the Atlantic near the surface and at depth. These outflows have a much tighter density class than the North Atlantic Deep Water inflow.

The circulation of the Southern Indian Ocean is quite complicated, as can be seen in Figure (6-9). With the exception of a rather large poleward surface flow, the circulation is characterized by a number of relatively small recirculations. The circulation equatorward of $30^{\circ} \mathrm{S}$ suggests a great deal of diapycnal flow and mixing .

The circulation of the South Pacific Ocean (Figure (6-10)) is no easier to interpret 


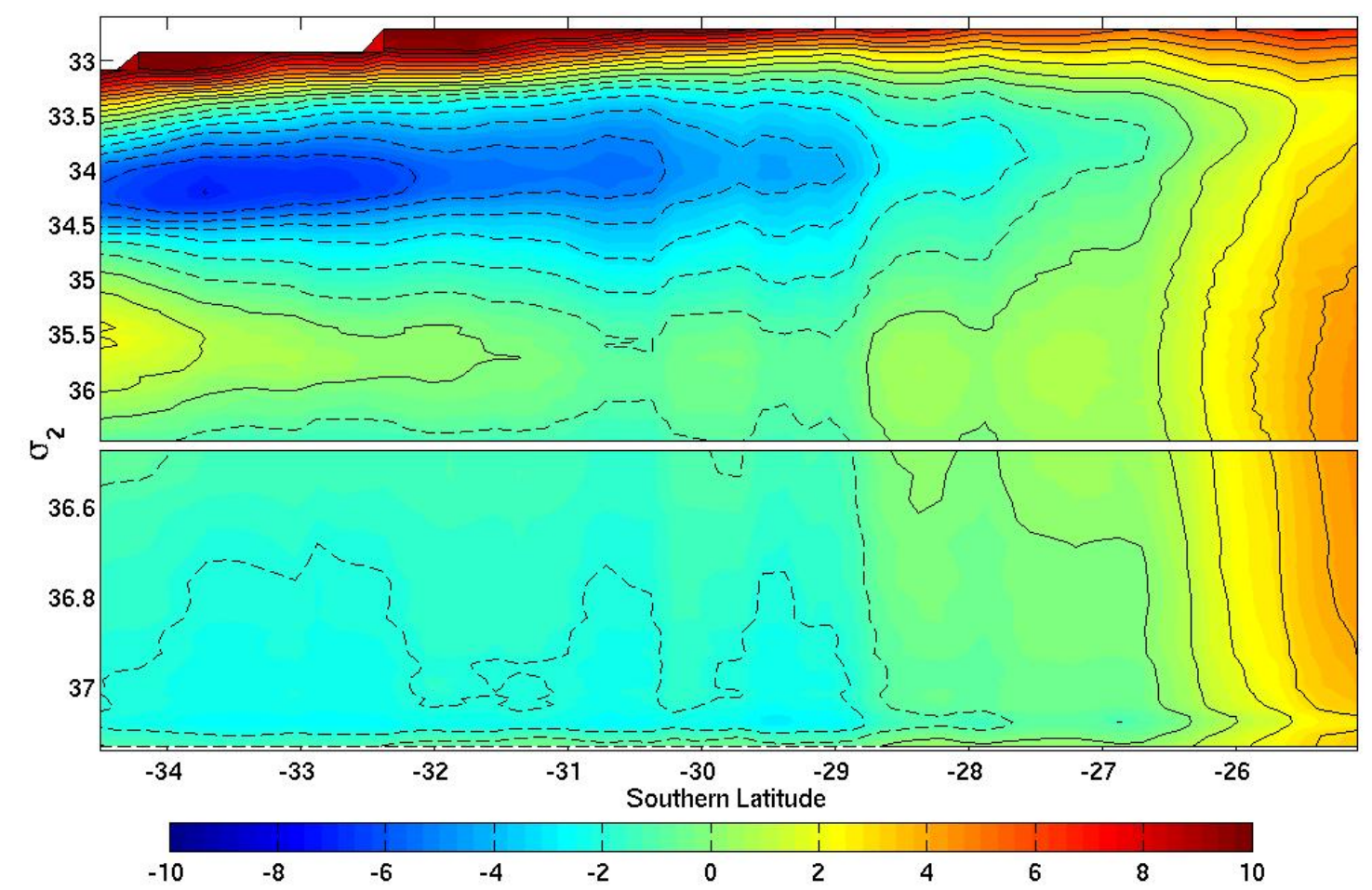

Figure 6-9: Temporal and zonal mean streamfunction calculated in isopycnal space for the South Indian Ocean. The contour interval is $1 \mathrm{~Sv}$.

than in the Indian Ocean. There is a surface cell of order 10 Sverdrups, which continually gains buoyancy as its waters move equatorward. The buoyancy source is most likely mixing from above with poleward flowing Ekman layer waters. A weaker flow beneath this cell is subject to several deep recirculations. While the sinking appears to happen primarily at, and equatorward of, $\sim 30^{\circ} \mathrm{S}$ in the South Indian Ocean, the sinking in the South Pacific appears to be at all latitudes shown in Figure (6-10). This is likely due to the fact that the latitude of the Subtropical Front is relatively constant $\left(\sim 40^{\circ} \mathrm{S}\right)$ over the South Indian Ocean. Over the South Pacific Ocean, however, the latitude of the Subtropical Front ranges from $\sim 45^{\circ} \mathrm{S}$ in the west to $\sim 30^{\circ} \mathrm{S}$ in the east(Orsi et al.; 1995). One can imagine an increase in mixing found at frontal locations. Since the South Pacific Ocean fronts exhibit large latitude shifts, it is to be expected that mixing is found across a large latitude range.

Figures (6-8), (6-9), and (6-10) are used to compare transport values with those from some of the most recent studies that infer transports from observations. The 


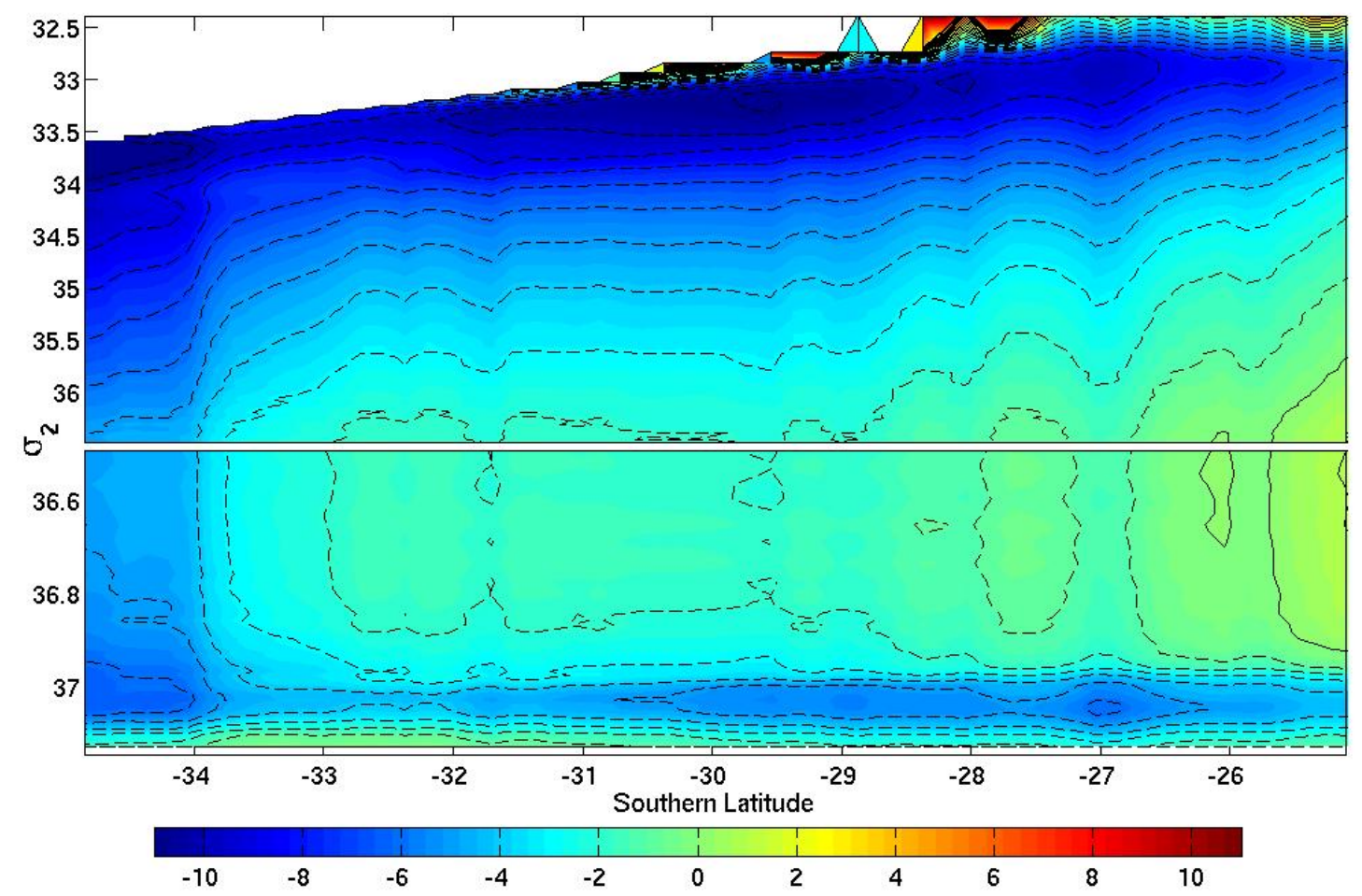

Figure 6-10: Temporal and zonal mean streamfunction calculated in isopycnal space for the South Pacific Ocean. The contour interval is $1 \mathrm{~Sv}$.

fluxes across $\sim 30^{\circ} \mathrm{S}$ given in these studies, and in the Southern Ocean state estimate, are given in Table (6.3). Transports from the Fine Resolution Antarctic Model (FRAM) (Saunders and Thompson; 1993) are also included in this table. Water class distinctions in the table (Ekman, thermocline water (TW), intermediate water (IW), upper deep water (UDW), lower deep water (LDW), and bottom water (BW) water) are non-uniform, and their associated density varies from study to study. This study finds $30^{\circ} \mathrm{S}$ to be a region of convergence (see Figure (6-7)) where poleward flowing surface waters meet slightly denser equatorward flowing surface waters and cause subduction. The complexity of dividing this region into separate water masses highlights the complexity of the Southern Ocean circulation. Nonetheless, an attempt is made such that a comparison between the state estimate flow with common modeled and observed overturning circulation patterns can be attained. For this study, the potential density of the water classes at $30^{\circ} \mathrm{S}$ are approximately: 


$$
\begin{aligned}
& \text { Ekman layer } \quad<32.6 \mathrm{~kg} / \mathrm{m}^{3} \\
& 32.6 \mathrm{~kg} / \mathrm{m}^{3}<\text { thermocline water }<33.2 \mathrm{~kg} / \mathrm{m}^{3} \\
& 33.2 \mathrm{~kg} / \mathrm{m}^{3}<\text { intermediate water }<33.6 \mathrm{~kg} / \mathrm{m}^{3} \\
& 33.6 \mathrm{~kg} / \mathrm{m}^{3}<\text { upper deep water }<36.5 \mathrm{~kg} / \mathrm{m}^{3} \\
& 36.5 \mathrm{~kg} / \mathrm{m}^{3}<\text { lower deep water }<37.0 \mathrm{~kg} / \mathrm{m}^{3} \\
& 37.0 \mathrm{~kg} / \mathrm{m}^{3}<\quad \text { bottom water }
\end{aligned}
$$

Again, these classes are approximations and distinctions will vary from basin to basin. Note that these cutoff points would have been different if this analysis had been done at a different latitude.

Loosely combining water classes in Table (6.1) allows a decent agreement between the observational inferences and the state estimate in the South Atlantic and South Pacific Ocean. For example, considering the Southern Ocean to Atlantic Ocean exchange, the observations imply about 8 to $20 \mathrm{~Sv}$ of the upper three water classes and about 0 to $7 \mathrm{~Sv}$ of bottom water leave the Southern Ocean to the Atlantic. These observations infer between 13 to $23 \mathrm{~Sv}$ enter the Southern Ocean as upper deep water. The state estimate gives $5 \mathrm{~Sv}$ of upper density class waters and about $7 \mathrm{~Sv}$ of bottom water $(\mathrm{LDW}+\mathrm{BW})$ leaving the Southern Ocean. These $12 \mathrm{~Sv}$ return as upper deep waters. Within the several Sverdrup margin of error expected for the non-converged state estimate, this is consistent. The Pacific Ocean exchange is similar, though the bottom water outflow is quite small. The Indian Ocean exchange is rather different, though this is not surprising as Figure (6-9) depicts a complex circulation where in-flowing and out-flowing water masses are not easily recognized.

It should be noted that open boundary controls are implemented, but only the temperature and salinity boundary conditions have thus far been adjusted. The total meridional transport out of the model domain is therefore dictated by the ECCOGODAE global state estimate prescribed boundary conditions. Besides this prescribed boundary condition, mass sources and sinks in the model are minimal. There 


\begin{tabular}{|c|c|c|c|c|c|}
\hline & & Observation & & & \\
\hline $\begin{array}{c}\text { Circulation at } \\
\sim 30^{\circ} \mathrm{S}\end{array}$ & $\begin{array}{c}\text { Talley et al. } \\
2003^{*}\end{array}$ & $\begin{array}{c}\text { Sloyan \& } \\
\text { Rintoul 2001a }\end{array}$ & $\begin{array}{l}\text { Ganachaud \& } \\
\text { Wunsch } 2000\end{array}$ & FRAM & SOSE \\
\hline Estimated error & \pm 3 to 5 & ? & & ?? & ? \\
\hline "Atlantic: $\quad$ Ekman & 0.99 & & & & 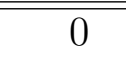 \\
\hline Atlantic: $\quad$ TW & 7.4 & 2 & & 13 & 3 \\
\hline Atlantic: $\quad$ IW & 5.2 & 8 & $16 \pm 3$ & 6 & 2 \\
\hline Atlantic: $\quad$ UDW & -178 & -7 & $-23+3$ & 205 & $\begin{array}{l}-12 \\
\end{array}$ \\
\hline Atlantic: LDW & -16.8 & -10 & $-2 J \pm 3$ & -20.5 & 2 \\
\hline Atlantic: $\quad$ BW & 3.8 & 6 & $6 \pm 1.3$ & 1.5 & 5 \\
\hline Ekman & 2.2 & & & & $\overline{-10}$ \\
\hline Indian: & -11.2 & -6 & & -11 & 12 \\
\hline Indian: & 107 & -8 & $27+6$ & & 3 \\
\hline Indian: & -10.8 & -20 & $-21 \pm 0$ & -4.5 & -5 \\
\hline Indian: $\quad$ LDW & 128 & 13 & $3 \pm 5$ & 10 & 2 \\
\hline Indian: & 13.8 & 10 & $8 \pm 4$ & 5.5 & -2 \\
\hline Ekman & -4.4 & & & & -12 \\
\hline Pacific: & 18 & 8 & $10+5$ & 8 & 22 \\
\hline Pacific: & 4.8 & 0 & $19 \pm 5$ & -4 & 1 \\
\hline Pacific: & -9.3 & -25 & $-9 \pm 3$ & -11 & -9 \\
\hline Pacific: & 138 & 18 & $7+2$ & 7 & 3 \\
\hline Pacific: & 13.8 & 8 & $1 \pm 2$ & $r$ & -5 \\
\hline Global: & $-1.3,-1.3^{*}$ & & & & -11 \\
\hline Global: & $-7.7,-8.0^{*}$ & 4 & & 10 & 30 \\
\hline Global: & $9.0,11.8^{*}$ & 0 & $35 \pm 8$ & -2.5 & 4 \\
\hline Global: & $-21.8,-30.3^{*}$ & -52 & $-59 \pm 12$ & -21.5 & -23 \\
\hline Global: & 2 127 2* & 21 & 2 & 1 & 9 \\
\hline Global: & $2 Z .1,21.0$ & 25 & $24 \pm 12.0$ & 14 & -9 \\
\hline
\end{tabular}

Table 6.1: Fluxes out of (positive) and into (negative) the Southern Ocean in Sverdrups. Water class distinctions are intentionally vague. The table is intended to allow comparison between the modeled and observed overturning circulation patterns. Only the most recent observational estimates were used in this table; many regional and older estimates were omitted (e.g. Bryden and Beal (2001); Macdonalds (1998); Robbins and Toole (1997); Schmitz (1995); Toole and Warren (1993)). *From Talley (2003): the first value uses Indian Ocean velocities from Robbins and Toole (1997), and the second value uses Indian Ocean velocities from Reid (2003). Fine Resolution Antarctic Model (FRAM) transports are from Saunders and Thompson (1993). SOSE denotes the Southern Ocean state estimate analyzed in this study. A "?" in the estimated error row denotes error bounds were not readily accessible. 
is a surface freshwater flux which allows evaporation and precipitation, and runoff is prescribed at land boundaries, but one expects the net effect of these sources to be insignificant compared to inter-ocean exchange. Therefore, to lowest order, the model is constrained to the lower resolution ECCO prescribed northern boundary conditions.

The prescribed northern boundary conditions are taken from the ECCO-GODAE $1^{o}$ state estimate. This is an improved solution to the global $2^{o}$ state estimate previously carried out by the ECCO consortium. The time mean horizontal transports were calculated from the $2^{o}$ state estimate, and it was found that the transports had converged to those given by independent box model inversions (Stammer et al.; 2003). It is therefore expected that the transports implied by the northern boundary conditions are of reasonable magnitudes. The structure of the prescribed transport, however, may be largely inconsistent with what an eddy-permitting state estimate would find. At the resolution of the model in this project, it is likely that additional transport pathways have become available, both through the resolution of small scale ocean dynamics, and through the use of more realistic topography. Open boundary controls should eventually bring the northern boundary condition into consistency with the interior. At this point in the work, however, this has not happened. A boundary condition that prescribes the proper inter-basin exchanges in a $1^{\circ}$ ECCO state estimate may not prescribe the proper exchanges when interpolated to be used in a $1 / 6^{\circ}$ resolution state estimate.

\subsection{Discussion}

How are water masses and their properties fluxed from one basin to another? We may envision two pathway regimes. The first path is by direct current flows, e.g. boundary currents or filaments of the Antarctic Circumpolar Current. This mechanism becomes apparent by looking at mean streamlines. The second inter-basin transport mechanism is transient eddy fluxes. The transient eddy transport is not along stream-

lines; this transport results from a breaking of streamline filaments causing fluid to 
move, possibly adiabatically, from one streamline to another. It is interesting that the meridional circulation in the Southern Ocean state estimate is, for the most part, weaker than observations imply, ${ }^{2}$ yet the zonal circulation is much stronger. Perhaps an inadequate representation of the latter transport mechanism (i.e. a poor representation of streamline instability processes) is preventing cross-frontal flows, and is thus responsible for both these transport anomalies.

The model may be more dynamically stable than the real ocean. Insight into how this model error may cause the transport discrepancies found above (too low meridional transport and too high zonal transport) is given by three numerical calculation carried out by Olbers and Eden (2003) . These three numerical models of the Southern Ocean all had the same wind stress applied at the surface, and thus they all had similar Ekman transport. The first experiment had an homogenous ocean and a flat bottom. The only way mass (and vorticity) could be balanced on circumpolar streamlines was through a frictional bottom boundary layer. In this scenario the transport of the Antarctic Circumpolar Current became much larger than observed (more than 600 Sverdrups), so that this bottom boundary layer could be effective. Olbers and Eden then introduced more realistic bathymetry. Now the meridional Ekman flux could be more easily balanced by deep western boundary currents. In this regime, where stretching of the water column (topographic $\beta$ ) and bottom torques are present, the Antarctic Circumpolar Current transport drops to approximately 30 Sv, a value much smaller than observed. In Olbers and Eden's last experement they added a density structure. In this regime a reasonable Antarctic Circumpolar Current transport was found. The conclusion is that the baroclinicity of the Southern Ocean is a key factor in determining the Antarctic Circumpolar Current transport. They found that vortex stretching within the water column plays a significant role in the Antarctic Circumpolar Current dynamics, allowing the current to deviate from $f / h$ contours and increasing its transport.

From this result it may be inferred that the strength of the fronts in the South-

\footnotetext{
${ }^{2}$ Compared to other studies, the meridional overturning in the Subpolar Zone is of reasonable magnitude.
} 
ern Ocean are a dominant factor in determining the Antarctic Circumpolar Current transport. This finding is substantiated by the work of Gent et al. (2001), which concludes that the Drake Passage Transport is set by the strength of the meridional Ekman transport at the latitude of the Drake Passage, and by the strength of the overturning circulation off the Antarctic shelf. One could infer that both these processes actually act to do the same thing, that is, they both increase the strength of the Polar Front. The strength of a front cannot increase without bounds, however; eventually instabilities will occur and break down the steep isopycnals. A balance occurs between surface forcing and instability processes. This balance determines the strength of the front, and thus the strength of both the along-front zonal transport, and the cross-front meridional transport. Model parameters are tuned to prevent numerical instabilities, and in doing so, may suppress physical instabilities of the flow. Theoretical barotropic and baroclinic instability criteria do not involve diffusive or viscous parameters (see Chapter 7 of Pedlosky (1987)), but that these model parameterizations do not damp out instability events is not obvious. The inferences gained from the work of Olbers and Eden (2003) imply that a model able to resolve strong fronts, yet built to suppress dynamical instabilities, is likely to find too high a zonal transport. The strong fronts in this model may also cause a weakened inter-basin exchange. Future work should investigate the instability processes occurring in the model and reevaluate the parameterization coefficients used.

The ECCO $1^{o}$ state estimate gives a reasonable Drake Passage transport. Three possible reasons for this are, first, that the ECCO state estimate does not resolve fronts as well as the Southern Ocean state estimate presented, and thus has a significantly different zonal flow structure. Second, the topography of the ECCO state estimate has been manually tuned (Lu et al.; 2002). Topography is very influential to circulation, especially in energetic regions like the Southern Ocean (Losch and Wunsch; 2003; Losch and Heimbach; 2006). Third, the choice of eddy parameterizations has been shown to have a great effect on the Drake Passage transport in numerical models (Gent et al.; 2002; Hallberg and Gnanadesikan; 2005). The Gent-McWilliams Redi coefficient in the ECCO state estimate is three order of magnitudes larger than 
that used in the Southern Ocean state estimate. It is not obvious which of these three factors is the dominant reason for the transport discrepancy. Incorporating bottom topography and the Gent-McWilliams Redi along-isopycnal mixing coefficient into the control vector would be the best way to address this issue. Future work should attempt to incorporate these controls.

A dominant feature of the meridional overturning streamfunctions plotted in Section 6.3 is a significant cross-isopycnal flow over most depths and latitudes (see for example Figure (6-7)). Significant diapycnal flow is confined to topographically complex boundary areas (Wunsch and Ferrari; 2004). A study by Garabato et al. (2004) suggests that not only is the Southern Ocean full of these boundary regions, but that there influence can span distances of thousands of kilometers. One concern this raises is that if boundary regions can influence remote areas, could unphysical processes occuring at the open boundary (at $24.7^{\circ} \mathrm{S}$ ) forcing the strong diapycnal flow noted near $28^{\circ} \mathrm{S}$ ? This concern aside, with the complex topography and strong flows in the Antarctic Circumpolar Current, the work of Garabato et al. (2004) implies that the results presented are feasible. Other observational studies have also inferred high diapycnal mixing rates in the Southern Ocean. (Polzin and Firing (1997) found large mixing near the Kerguelen Plateau caused by interactions of the Antarctic Circumpolar Current with topography, and Heywood et al. (2002) inferred large mixing in the Weddell Sea from heat budgets.) The streamfunctions presented in Section 6.3 are zonal integrals; future work must determine which regions contribute to the strong diapycnal flow found in the Southern Ocean state estimate.

\subsection{Conclusion}

A high-resolution state estimate of the Southern Ocean has been produced. As discussed in Chapter 5, this state estimate has yet to reach what is deemed complete consistency with observations. Analysis of this non-converged solution, however, has been insightful. More than drawing conclusions, this work has raised questions.

A strong temporal correlation with a negligible time lag is found in the zonal 
transport across meridional sections. It is hypothesized that this is caused by atmospheric forcing. Wind stress curl and buoyancy forcing combine to setup fronts in Southern Ocean. Barotropic and baroclinic instabilities occur, redistributing buoyancy and breaking down the fronts. A balance occurs between these processes and sets the strength of the Antarctic Circumpolar Current. Thus wind and buoyancy forcing should be correlated with both the meridional redistribution of buoyancy and the zonal volume transport. Future work should try to unravel these correlations.

Dense waters, $37.05 \mathrm{~kg} \mathrm{~m}^{-3} \leq \sigma_{2} \leq 37.2 \mathrm{~kg} \mathrm{~m}^{-3}$, are found at the northern boundary of the domain. Plots of the meridional streamfunction (Figure (6-7)) show that there is no clear path of this water from formation sites, which are located primarily in the Ross and Weddel Seas, to this boundary. Besides determining what drives the formation of the deep water, future work should try to determine how this water reaches the subtropical oceans.

One discrepancy between the state estimate and previous work on Southern Ocean transport is in the flow of bottom water, The state estimate finds a much lower outflow into the South Indian and South Pacific Oceans than what was implied from observations. What is alarming about the state estimate solution in these regions is the recirculation of bottom waters where denser water is found to flow poleward. It appears that as deep water flows north, much of it loses buoyancy, sinks, and returns. This seems unphysical, one imagines deep water gaining buoyancy as it flows equatorward and mixes with buoyant waters above it. A likely cause of this circulation is topographic interaction. If deep water pathways are blocked, the water must recirculate, and this recirculation may occur over a very large area in the horizontal plane. The deep water circulation features plotted are likely more of meanderings between basins than an overturning. It is possible that the flow pattern may appear inverted in the zonal average, even when careful care was taken to make the average along isopycnals. This emphasizes, once again, that the Southern Ocean circulation is complex, and needs to be considered in all three spatial dimensions. Future work must describe the Southern Ocean volume flux in all spatial dimensions. This description should also include an accounting of where across-isopycnal mixing is significant. Prelimi- 
nary work suggests comparing flow along isopycnal sections with zonally integrated sections can provide great insight to the pathways of transport. Future work should also separate water masses more rigorously through analysis of their temperature and salinity properties.

Much of what is known about the Southern Ocean comes from numerical models (e.g.FRAM) that are not rigorously compared or constrained to data. This work marks a step towards determining a model state of the Southern Ocean that is consistent with data. There is still much work to be done in both progressing the state estimate towards a more acceptable solution, and in answering the many questions raised throughout this thesis. 


\section{Appendix A}

\section{Formulation of the Residual Mean}

\section{Circulation}

A formulation for calculating the residual mean streamfunction in vertical coordinates was derived for temporally constant flows by McIntosh and McDougal (1996). Lee and Coward (2003) appended a temporal dependence to this derivation. A more complete derivation of this formulation is given below.

The goal is to find the zonal integral of the time mean flux of a property, $C$, between two isopycnal layers, $\rho_{1}$ and $\rho_{2}$, of depth $z_{1}$ and $z_{2}$ :

$$
\int_{x_{W}}^{x_{E}} \frac{1}{\tau} \int_{0}^{\tau} \int_{z_{2}\left(x, y, \rho_{2}, t\right)}^{z_{1}\left(x, y, \rho_{1}, t\right)} v C d z d t d x
$$

where $v$ is the meridional velocity, $\tau$ is the duration of the analysis period, and $C$ is any fluid property in question (e.g. heat content). To determine the volume transport between the isopycnals one would set $C=1$.

To gain insight into the processes at work in this transport, variables are separated into mean components and perturbations from the means: $v=\bar{v}+v^{\prime}=[\bar{v}]+\bar{v}^{*}+v^{\prime}$. Here an overbar denotes a time mean, a square bracket denotes a zonal mean, a prime denotes a deviation from the time mean, and an asterisk denotes a deviation from the zonal mean. This is also done for the isopycnal depth such that $z_{n}\left(x, y, \rho_{n}, t\right)=$ $\overline{z_{n}}+z_{n}^{\prime}=\left[\overline{z_{n}}\right]+{\overline{z_{n}}}^{*}+z_{n}^{\prime}$. In the following calculation the assumption is made that 
perturbation values are of higher order than the mean. That is, while barred and bracketed variables are considered $\mathrm{O}(1)$, primed and starred variables are considered $\mathrm{O}(\epsilon)$, where $\epsilon<<1$. The following calculation will be carried out to second order; all terms of $\mathrm{O}\left(\epsilon^{3}\right)$ will be neglected. The validity of this approximation is discussed below.

Separating the time mean and anomaly in Equation (1.1), the first integral to evaluate gives

$$
\int_{\overline{z_{2}}+z_{2}^{\prime}}^{\overline{z_{1}}+z_{1}^{\prime}} v C d z=\int_{\overline{z_{2}}}^{\overline{z_{1}}} v C d z+\int_{\overline{z_{1}}}^{\overline{z_{1}}+z_{1}^{\prime}} v C d z-\int_{\overline{z_{2}}}^{\overline{z_{2}}+z_{2}^{\prime}} v C d z
$$

A time mean of this integral must be taken. For the first term on the right hand side of Equation (1.2), this is simply

$$
\frac{1}{\tau} \int_{0}^{\tau} \int_{\overline{z_{1}}}^{\overline{z_{2}}} v C d z d t=\int_{\overline{z_{1}}}^{\overline{z_{2}}}\left(\overline{v C}+\overline{v^{\prime} C^{\prime}}\right) d z
$$

Since $z^{\prime}$ denotes a small deviation from $\bar{z}$, the last two terms on the right hand side of Equation (1.2) can be evaluated by a Taylor expansion about $\bar{z}$. The Taylor expansion need only be carried out to second order as the integral is $\mathrm{O}(\epsilon)$ and, as stated above, $\mathrm{O}\left(\epsilon^{3}\right)$ terms are neglected. Thus the last two terms can be written as

$$
\begin{aligned}
\frac{1}{\tau} \int_{0}^{\tau} \int_{\overline{z_{n}}}^{\overline{z_{n}}+z_{n}^{\prime}}(v C) d z d t & =\left.\frac{1}{\tau} \int_{0}^{\tau} \int_{\overline{z_{n}}}^{\overline{z_{n}}+z_{n}^{\prime}}(v C)\right|_{\overline{z_{n}}}+\left.(v C)_{z}\right|_{\overline{z_{n}}}\left(z-\overline{z_{n}}\right)+\cdots d z d t \\
& =\overline{\left.(v C)\right|_{\overline{z_{n}}} z_{n}^{\prime}}+(1 / 2) \overline{\left.(v C)_{z}\right|_{\overline{z_{n}}} z_{n}^{\prime 2}}+O\left(\epsilon^{3}\right) \\
& =\left.\left(\bar{C} \overline{v^{\prime} z^{\prime}}+\bar{v} \overline{C^{\prime} z^{\prime}}+(1 / 2)(\bar{v} \bar{C})_{z} \overline{z^{\prime 2}}\right)\right|_{\overline{z_{n}}} .
\end{aligned}
$$

Using the equation above, Equation 1.2 becomes, to $\mathrm{O}\left(\epsilon^{2}\right)$,

$$
\begin{aligned}
\frac{1}{\tau} \int_{0}^{\tau} \int_{\overline{z_{2}}+z_{2}^{\prime}}^{\overline{z_{1}}+z_{1}^{\prime}} v C d z d t= & \int_{\overline{z_{1}}}^{\overline{z_{2}}}\left(\overline{v C}+\overline{v^{\prime} C^{\prime}}\right) d z+ \\
& \left.\left(\bar{C} \overline{v^{\prime} z^{\prime}}+\bar{v} \overline{C^{\prime} z^{\prime}}+(1 / 2)(\bar{v} \bar{C})_{z} \overline{z^{\prime 2}}\right)\right|_{\overline{z_{2}}} ^{\overline{z_{1}}}
\end{aligned}
$$


Now, in accordance with Equation (1.1), the zonal integral of Equation 1.5 is taken.

$$
\begin{aligned}
\int_{x_{W}}^{x_{E}} \frac{1}{\tau} \int_{0}^{\tau} \int_{\overline{z_{2}}+z_{2}^{\prime}}^{\overline{z_{1}}+z_{1}^{\prime}} v C d z d t d x= & \int_{x_{W}}^{x_{E}} \int_{\overline{z_{1}}}^{\overline{z_{2}}}\left(\overline{v C}+\overline{v^{\prime} C^{\prime}}\right) d z d x \\
& +\left.\int_{x_{W}}^{x_{E}}\left(\bar{C} \overline{v^{\prime} z^{\prime}}+\bar{v} \overline{C^{\prime} z^{\prime}}+(1 / 2)(\bar{v} \bar{C})_{z} \overline{z^{\prime 2}}\right)\right|_{\overline{z_{2}}} ^{\overline{z_{1}}} d x
\end{aligned}
$$

Zonal mean perturbations of time mean anomaly perturbations are neglected as these are higher order.

$$
\begin{aligned}
\int_{x_{W}}^{x_{E}} \frac{1}{\tau} \int_{0}^{\tau} \int_{\overline{z_{2}}+z_{2}^{\prime}}^{\overline{z_{1}}+z_{1}^{\prime}} v C d z d t d x= & \int_{x_{W}}^{x_{E}} \int_{\overline{z_{1}}}^{\overline{z_{2}}}\left(\overline{v C}+\overline{v^{\prime} C^{\prime}}\right) d z d x \\
& +L\left[\left.\left(\bar{C} \overline{v^{\prime} z^{\prime}}+\bar{v} \overline{C^{\prime} z^{\prime}}+(1 / 2)(\bar{v} \bar{C})_{z} \overline{z^{\prime 2}}\right)\right|_{\overline{z_{2}}} ^{\overline{z_{1}}}\right]
\end{aligned}
$$

Again, [ ], denotes a zonal mean, and thus $L[]$, where $\mathrm{L}$ is the length of the integration path, is the zonal integral. The fact that $L$ is a function of $z$ can be overlooked, as the calculation to be made in this work will be in the limit where $z_{1} \rightarrow z_{2}$, and thus $L$ can be approximated as constant in depth.

The first term on the right hand side of Equation (1.7) is evaluated analogously to the time mean calculation. This term is seperated into zonal mean and perturbation components such that the vertical integral becomes, to $\mathrm{O}\left(\epsilon^{2}\right)$,

$$
\begin{aligned}
\int_{\left[\overline{z_{2}}\right]+\overline{z_{2}} *}^{\left[\overline{z_{1}}\right]+\overline{z_{1}} *}\left(\bar{v} \bar{C}+\overline{v^{\prime} C^{\prime}}\right) d z= & \int_{\left[\overline{z_{2}}\right]}^{\left[\overline{z_{1}}\right]}\left(\bar{v} \bar{C}+\overline{v^{\prime} C^{\prime}}\right) d z \\
& +\int_{\left[\overline{z_{1}}\right]}^{\left[\overline{z_{1}}\right]+\overline{z_{1}} *}(\bar{v} \bar{C}) d z-\int_{\left[\overline{z_{2}}\right]}^{\left[\overline{z_{2}}\right]+\overline{z_{2}} *}(\bar{v} \bar{C}) d z .
\end{aligned}
$$

A zonal integral of the first term on the right hand side of Equation (1.8) gives

$$
\int_{x_{W}}^{x_{E}} \int_{\left[\overline{z_{2}}\right]}^{\left[\overline{z_{1}}\right]}\left(\bar{v} \bar{C}+\overline{v^{\prime} C^{\prime}}\right) d z d x=\int_{\left[\overline{z_{2}}\right]}^{\left[\overline{z_{1}}\right]} L\left([\bar{v} \bar{C}]+\left[\bar{v}^{*} \bar{C}^{*}\right]+\left[\overline{v^{\prime} C^{\prime}}\right]\right) d z
$$

A Taylor expansion is used to evaluate the last two terms of Equation (1.8), and 
shows

$$
\begin{aligned}
\int_{x_{W}}^{x_{E}} \int_{\left[\overline{z_{n}}\right]}^{\left[\overline{z_{n}}\right]+{\overline{z_{n}}}^{*}}(\bar{v} \bar{C}) d z d x & =\left.\int_{x_{W}}^{x_{E}} \int_{\left[\overline{z_{n}}\right]}^{\left[\overline{z_{n}}\right]+{\overline{z_{n}}}^{*}}(\bar{v} \bar{C})\right|_{\left[\overline{z_{n}}\right]}+\left.(\bar{v} \bar{C})_{z}\right|_{\left[\overline{z_{n}}\right]}\left(\bar{z}-\left[\overline{z_{n}}\right]\right)+\cdots d z d x \\
& =\left.\int_{x_{W}}^{x_{E}}(\bar{v} \bar{C})\right|_{\left[\bar{z}_{n}\right]}{\overline{z_{n}}}^{*}+\left.(1 / 2)(\bar{v} \bar{C})_{z}\right|_{\left[\bar{z}_{n}\right]}{\overline{z_{n}}}^{* 2} d x \\
& =\left.L\left([\bar{v}]\left[\bar{C}^{*} \bar{z}^{*}\right]+[\bar{C}]\left[\bar{v}^{*} \bar{z}^{*}\right]+(1 / 2)\left[(\bar{v} \bar{C})_{z}\right] \bar{z}^{* 2}\right)\right|_{\left[\overline{z_{n}}\right]}
\end{aligned}
$$

using Equation (1.9), Equation (1.1) has become

$$
\begin{aligned}
\int_{x_{W}}^{x_{E}} \frac{1}{\tau} \int_{0}^{\tau} \int_{z_{2}}^{z_{1}} v C d z d t d x= & \int_{\left[\overline{z_{2}}\right]}^{\left[\overline{z_{1}}\right]} L\left([\bar{v} \bar{C}]+\left[\bar{v}^{*} \bar{C}^{*}\right]+\left[\overline{\left.v^{\prime} C^{\prime}\right]}\right) d z\right. \\
& \left.L\left([\bar{v}]\left[\bar{C}^{*} \bar{z}^{*}\right]+[\bar{C}]\left[\bar{v}^{*} \bar{z}^{*}\right]+(1 / 2)\left[(\bar{v} \bar{C})_{z}\right] \bar{z}^{* 2}\right)\right|_{\left[\overline{z_{2}}\right]} ^{\left[\overline{z_{1}}\right]} \\
& +L\left[\left.\left(\bar{C} \overline{v^{\prime} z^{\prime}}+\bar{v} \overline{C^{\prime} z^{\prime}}+(1 / 2)(\bar{v} \bar{C})_{z} \overline{z^{\prime 2}}+\right)\right|_{\overline{z_{2}}} ^{\overline{z_{1}}}\right]+O\left(\epsilon^{3}\right),
\end{aligned}
$$

Now define $h \equiv z_{1}-z_{2}=\left[\overline{z_{1}}\right]+{\overline{z_{1}}}^{*}+z_{1}^{\prime}-\left[\overline{z_{2}}\right]-{\overline{z_{2}}}^{*}-z_{2}^{\prime}$. Note that $\bar{h}=$ $\left[\overline{z_{1}}\right]+{\overline{z_{1}}}^{*}-\left[\overline{z_{2}}\right]-{\overline{z_{2}}}^{*}$ and then $[\bar{h}]=\left[\overline{z_{1}}\right]-\left[\overline{z_{2}}\right]$. Keeping $h$ finite, but taking the limit where $\rho_{1}\left(x, y, z_{1}, t\right)$ approaches $\rho_{2}\left(x, y, z_{2}, t\right)$, such that $z_{1} \approx z_{2} \approx z$, Equation (1.11) becomes

$$
\begin{aligned}
\int_{x_{W}}^{x_{E}} \frac{1}{\tau} \int_{0}^{\tau} \int_{z_{2}}^{z_{1}} v C d z d t d x= & L[\bar{h}]\left([\bar{v}][\bar{C}]+\left[\bar{v}^{*} \bar{C}^{*}\right]+\left[\overline{v^{\prime} C^{\prime}}\right]\right) \\
& L[\bar{h}] \frac{\partial}{\partial[\bar{z}]}\left([\bar{v}]\left[\bar{C}^{*} \bar{z}^{*}\right]+[\bar{C}]\left[\bar{v}^{*} \bar{z}^{*}\right]+(1 / 2)\left[(\bar{v} \bar{C})_{z}\right] \bar{z}^{* 2}\right) \\
& +L\left[\bar{h} \frac{\partial}{\partial \bar{z}}\left(\bar{C} \overline{v^{\prime} z^{\prime}}+\bar{v} \overline{C^{\prime} z^{\prime}}+(1 / 2)(\bar{v} \bar{C})_{z} \overline{z^{\prime 2}}\right)\right]
\end{aligned}
$$


The no slip boundary condition imposed in the model allows one useful simplification to be made to the last term in Equation (1.11).

$$
\begin{aligned}
L\left[\overline { h } \frac { \partial } { \partial \overline { z } } \left(\bar{C} \overline{v^{\prime} z^{\prime}}+\bar{v}\right.\right. & \left.\left.\overline{C^{\prime} z^{\prime}}+(1 / 2)(\bar{v} \bar{C})_{z} \overline{z^{\prime 2}}\right)\right] \\
= & \int_{x_{W}}^{x_{E}}\left(\bar{h} \frac{\partial}{\partial \bar{z}}\right)\left(\bar{C} \overline{v^{\prime} z^{\prime}}+\bar{v} \overline{C^{\prime} z^{\prime}}+(1 / 2)(\bar{v} \bar{C})_{z} \overline{z^{\prime 2}}\right)_{z} d x \\
= & {[\bar{h}] \frac{\partial}{\partial[\bar{z}]} \int_{x_{W}}^{x_{E}}\left(\bar{C} \overline{v^{\prime} z^{\prime}}+\bar{v} \overline{C^{\prime} z^{\prime}}+(1 / 2)(\bar{v} \bar{C})_{z} \overline{z^{\prime 2}}\right) d x } \\
& +\left.\left(\bar{C} \overline{v^{\prime} z^{\prime}}+\bar{v} \overline{C^{\prime} z^{\prime}}+(1 / 2)(\bar{v} \bar{C})_{z} \overline{z^{\prime 2}}\right)\right|_{x_{E}} \frac{\partial x_{E}}{\partial z} \\
& -\left.\left(\bar{C} \overline{v^{\prime} z^{\prime}}+\bar{v} \overline{C^{\prime} z^{\prime}}+(1 / 2)(\bar{v} \bar{C})_{z} \overline{z^{\prime 2}}\right)\right|_{x_{W}} \frac{\partial x_{W}}{\partial z} \\
= & L[\bar{h}] \frac{\partial}{\partial[\bar{z}]}\left[\bar{C} \overline{v^{\prime} z^{\prime}}+\bar{v} \overline{C^{\prime} z^{\prime}}+(1 / 2)(\bar{v} \bar{C})_{z} \overline{z^{\prime 2}}\right]
\end{aligned}
$$

Where it was assumed that $\bar{h}^{*}$ is higher order so that only the term multiplied by $[\bar{h}]$ was retained. The boundary terms arising from the above application of the Leibniz integral rule are both zero, as $\bar{v}$ and $v^{\prime}$ are both identically zero on the boundaries. Using Equation 1.13, Equation (1.12) becomes

$$
\begin{aligned}
\int_{x_{W}}^{x_{E}} \frac{1}{\tau} \int_{0}^{\tau} \int_{z_{2}}^{z_{1}} v C d z d t d x= & L[\overline{h v C}] \\
= & L[\bar{h}]\left([\bar{v}][\bar{C}]+\left[\bar{v}^{*} \bar{C}^{*}\right]+\left[\overline{v^{\prime} C^{\prime}}\right]\right) \\
& +L[\bar{h}]\left([\bar{v}]\left[\bar{C}^{*} \bar{z}^{*}\right]+[\bar{C}]\left[\bar{v}^{*} \bar{z}^{*}\right]+(1 / 2)\left[(\bar{v} \bar{C})_{z}\right] \bar{z}^{* 2}\right)_{z} \\
& +L[\bar{h}]\left[\bar{C} \overline{v^{\prime} z^{\prime}}+\bar{v} \overline{C^{\prime} z^{\prime}}+(1 / 2)(\bar{v} \bar{C})_{z} \overline{z^{\prime 2}}\right]_{z}
\end{aligned}
$$

By dividing by $[\bar{h}]$ the volume weighted transport of $C$ is derived. In other words, 
if $z_{1}$ and $z_{2}$ represent the top and bottom of an isopycnal layer, such that $h$ is the isopycnal thickness, then

$$
\begin{aligned}
L[\overline{h v C}] /[\bar{h}]= & \\
& L\left([\bar{v}][\bar{C}]+\left[\bar{v}^{*} \bar{C}^{*}\right]+\left[\overline{v^{\prime} C^{\prime}}\right]\right) \\
& +L\left([\bar{v}]\left[\bar{C}^{*} \bar{z}^{*}\right]+[\bar{C}]\left[\bar{v}^{*} \bar{z}^{*}\right]+(1 / 2)\left[(\bar{v} \bar{C})_{z}\right] \bar{z}^{* 2}\right)_{z} \\
& +L\left[\bar{C} \overline{v^{\prime} z^{\prime}}+\bar{v} \overline{C^{\prime} z^{\prime}}+(1 / 2)(\bar{v} \bar{C})_{z} \overline{z^{\prime 2}}\right]_{z}
\end{aligned}
$$

is the time mean and zonally integrated average meridional transport of $C$ in the isopycnal layer. A more practical, and perhaps more insightful, formulation comes from a change of variables, $z \rightarrow \rho$. A Taylor expansion shows

$$
z_{n}^{\prime}=-\rho^{\prime} / \overline{\rho_{z}}+O\left(\epsilon^{2}\right),
$$

and

$$
{\overline{z_{n}}}^{*}=-\bar{\rho}^{*} /\left[\overline{\rho_{z}}\right]+O\left(\epsilon^{2}\right) .
$$

$$
\begin{aligned}
L[\overline{h v C}] /[\bar{h}]= & L\left([\bar{v}][\bar{C}]+\left[\bar{v}^{*} \overline{C^{*}}\right]+\left[\overline{v^{\prime} C^{\prime}}\right]\right) \\
+ & L /\left[\overline{\rho_{z}}\right]\left(-[\bar{v}]\left[\bar{C}^{*} \bar{\rho}^{*}\right]-[\bar{C}]\left[\bar{v}^{*} \bar{\rho}^{*}\right]+(1 / 2)\left[(\bar{v} \bar{C})_{z}\right] \bar{\rho}^{* 2}\right)_{z} \\
& +L\left[-\bar{C} \overline{v^{\prime} \rho^{\prime}} / \overline{\rho_{z}}-\bar{v} \overline{C^{\prime} \rho^{\prime}} / \overline{\rho_{z}}+(1 / 2)(\bar{v} \bar{C})_{z} \overline{\rho^{\prime 2}} / \overline{\rho_{z}}\right]_{z} .
\end{aligned}
$$

The equation above can be used to analyze meridional transport in the Southern Ocean state estimate. The only assumption made in its derivation is that perturbations from the mean are small. When the vertical density gradient approaches zero this assumption is violated. As can be seen from Equations (1.16) and (1.17), when $\rho_{z}$ is small, perturbations in the layer thickness can be of very large amplitude. It is to be expected that the analysis below will be robust over much of the ocean. There 
are two regions in the Southern Ocean state estimate domain, however, where $\partial \rho / \partial z$ is very small and the formulation may break down. These two regions are near the Antarctic Continent (especially in the Ross and Weddell Seas), and in the surface mixed layer. In these regions caution must be taken in evaluating the meridional transport with this formulation.

For analysis of the volume transport $C$ is set to 1 ,

$$
\begin{aligned}
L[\overline{h v}] /[\bar{h}]= & L[\bar{v}] \\
& +L /\left[\overline{\rho_{z}}\right]\left(-\left[\bar{v}^{*} \bar{\rho}^{*}\right]+(1 / 2)\left[(\bar{v})_{z}\right] \bar{\rho}^{* 2}\right)_{z} \\
& +L\left[-\overline{v^{\prime} \rho^{\prime}} / \overline{\rho_{z}}+(1 / 2)(\bar{v})_{z} \overline{\rho^{\prime 2}} / \overline{\rho_{z}}\right]_{z}
\end{aligned}
$$

and an additional simplification can be made. Thermal wind shows that a zonal mean of $v_{z}$ is of perturbation amplitude since

$$
\int_{x_{W}}^{x_{E}} v_{z} d x \approx \frac{-g}{f \rho_{o}} \int_{x_{W}}^{x_{E}} \rho_{x} d x=\left.\frac{-g}{f \rho_{o}} \rho^{*}\right|_{x_{W}} ^{x_{E}}
$$

making the third term on the right hand side of Equation (1.19) $\mathrm{O}\left(\epsilon^{3}\right)$. Therefore this term should be neglected. Equation (1.19) becomes

$$
\begin{aligned}
& L[\overline{h v}] /[\bar{h}]=\quad L[\bar{v}] \\
& -L\left[\bar{v}^{*} \bar{\rho}^{*}\right]_{z} /\left[\overline{\rho_{z}}\right] \\
& +L\left[-\overline{v^{\prime} \rho^{\prime}} / \overline{\rho_{z}}+(1 / 2)(\bar{v})_{z} \overline{\rho^{\prime 2}} / \overline{\rho_{z}}\right]_{z} .
\end{aligned}
$$

This equation is given as Equation 6.2 of Section 6.3. The reader is referred to this Section for a physical interpretation of these terms as well as a recasting of 1.21 into the context of an overturning streamfunction. 


\section{Bibliography}

Adcroft, A., Campin, J.-M., Heimbach, P., Hill, C. and Marshall, J. (2006). Mitgcm user's manual, (online documentation), MIT / EAPS, Cambridge, MA 02139, USA. http://mitgcm.org/sealion/online_documents/manual.html.

Arakawa, A. and Lamb, V. (1977). Computational design of the basic dynamical processes of the ucla general circulation model, Methods in Computational Physics 17: $174-267$.

Ayoub, N. (2006). Estimation of boundary values in a north atlantic circulation model using an adjoint method, Ocean Modelling 12: 319-347.

Bryden, H. and Beal, L. (2001). Role of the agulhas current in indian ocean circulation and associated heat and freshwater fluxes, Deep-Sea Research Part I 48(8): 18211845.

Conkright, M. E., Locarnini, R., Garcia, H. E., O’Brien, T., Boyer, T., Stephens, C. and Antonov, J. (2002). World ocean atlas 2001: Objective analyses, data statistics, and figures,cd-rom documentation, Technical report, Natl. Oceanogr.Data Cent., Silver Spring,Md.

Cunningham, S. A., Alderson, S. G., King, B. A. and Brandon, M. (2003). Transport and variability of the antarctic circumpolar current in drake passage, J. Geophys. Res. 108(C5).

Döös, K. and Webb, D. (1994). The deacon cell and the other meridional cells of the southern ocean, J. Phys. Oceanogr. 24: 429-442. 
Fekete, B. M., Vöröosmarty, C. J. and Grabs, W. (2002). High-resolution fields of global runoff combining observed river discharge and simulated water balances, Global Biogeochemical Cycles, 16(3).

Ferreira, D., Marshall, J. and Heimbach, P. (2005). Estimating eddy stresses by fitting dynamics to observations using a residual-mean ocean circulation model and its adjoint, Journal of Physical Oceanography 35(10): 1891-1910.

Forget, G. and Wunsch, C. (2006). Estimated global hydrographic variability, Journal of Physical Oceanography submitted.

Ganachaud, A. and Wunsch, C. (2000). Improved estimates of global ocean circulation, heat transport and mixing from hydrographic data, Nature 408(6811): 453457.

Ganachaud, A. and Wunsch, C. (2003). Large-scale ocean heat and freshwater transports during the world ocean circulation experiment, Journal of Climate 16.

Garabato, A. C. N., Polzin, K. L., King, B. A., Heywood, K. J. and Visbeck, M. (2004). Widespread intense turbulent mixing in the southern ocean, Science 303: $210-213$.

Gebbie, G. (2004). Subduction in an Eddy-resolving State Estimate of the Northeast Atlantic Ocean, PhD thesis, Massachusetts Institute of Technology / Woods Hole Oceanographic Institution Joint Program in Oceanography.

Gebbie, G. A., Heimbach, P. and Wunsch, C. (2005). Strategies for nested and eddyresolving state estimation. Submitted for publication.

Gent, P. R., Craig, A. P., Bitz, C. M. and Weatherly, J. W. (2002). Parameterization improvements in an eddy-permitting ocean model for climate, Journal of Climate 15(12): 1447-1459.

Gent, P. R., Large, W. G. and Bryan, F. O. (2001). What sets the mean transport through drake passage?, Journal of Geophysical Research, 106(C2): 2693-2712. 
Giering, R. and Kaminski, T. (1998). Recipes for adjoint code construction, ACM Trans. on Math. Software 24(4): 437-474.

Gilbert, J. C. and Lemaréchal, C. (1989). Some numerical experiments with variablestorage quasi-Newton algorithms, Math. Program. 45: 407-435.

Gill, P. E., Murray, W. and Wright, M. H. (1986). Practical Optimization, Academic Press, San Diego, CA.

Gille, S. T. (1995). Dynamics of the Antarctic Circumpolar Current: Evidence for Topographic Effects from Altimeter Data and Numerical Model Output, PhD thesis, MIT-WHOI Joint Program, Cambridge MA.

Gille, S. T. (1997). The southern ocean momentum balance: Evidence for topographic effects from numerical model output and altimeter data, J. Phys. Oceanogr. 27: 2219-2232.

Gould, J. and the Argo Steering Team (2004). Argo profiling floats bring new era of in situ ocean observations, EOS Trans. Am. Geophys. Un. 85(19): 190-191.

Gouretski, V. V. and Koltermann, K. P. (2004). Woce global hydrographic climatology, Technical report, Berichte des Bundesamt für Seeschifffahrt und Hydrographie Nr. 35/2004. Available at http://www.bsh.de/de/Produkte/Buecher/Berichte/index.jsp.

Hallberg, R. and Gnanadesikan, A. (2005). The role of eddies in determining the structure and response of the wind-driven southern hemisphere overturning: Results from the modeling eddies in the southern ocean project, Submitted to J. Phys. Oceanogr. .

Heimbach, P., Hill, C. and Giering, R. (2005). An efficient exact adjoint of the parallel mit general circulation model, generated via automatic differentiation, Future Generation Computer Systems (FGCS) 21(8): 1356-1371. doi:10.1016/j.future.2004.11.010. 
Heimbach, P., Ponte, R., Evangelinos, C., Forget, G., Mazloff, M., Menemenlis, D., Vinogradov, S. and Wunsch, C. (2006). Combining altimetric and all other data with a general circulation model, 15 Years of Progress in Radar Altimetry Symposium, Vol. SP-614, ESA Special Publication.

Heywood, K. J., Garabato, A. C. N., and Stevens, D. P. (2002). High mixing rates in the abyssal southern ocean, Nature 415: 1011-1014.

Hughes, C. W., Meredith, M. P. and Heywood, K. J. (1999). Wind-driven transport fluctuations through drake passage: A southern mode, Journal of Physical Oceanography 29(8): 1971-1992.

Hughes, C. W., Woodworth, P. L., Meredith, M. P., Stepanov, V., Whitworth, T. and Pyne, A. R. (2003). Coherence of antarctic sea levels, southern hemisphere annular mode, and flow through drake passage, Geophysical Research Letters 30(9).

Johnson, G. C. and Bryden, H. L. (1989). On the size of the antarctic circumpolar current, Deep-Sea Research 36: 39-53.

Kalnay, E. and coauthors (1996). The NCEP/NCAR 40-year reanalysis project, Bull. Amer. Meteor. Soc. 77: 431-437.

Large, W. G., McWilliams, J. C. and Doney, S. (1994). Oceanic vertical mixing: A review and a model with a nonlocal boundary layer parameterization, Rev. Geophysics 32: 363 .

Large, W. and Pond, S. (1982). Sensible and latent-heat flux measurements over the ocean, Journal of Physical Oceanography 12(5): 464-482.

Lee, M.-M. and Coward, A. (2003). Eddy mass transport for the southern ocean in an eddy-permitting global ocean model, Ocean Modelling 5(3): 249-266.

Levitus, S., Burgett, R. and Boyer, T. (2001). World ocean atlas 2001, Technical report, U.S. Department of Commerce, Washington, D.C. 
Losch, M. and Heimbach, P. (2006). Adjoint sensitivity of an ocean general circulation model to bottom topography, J. Phys. Oceanogr. In press.

Losch, M. and Wunsch, C. (2003). Bottom topography as a control variable in an ocean model, Journal of Atmospheric and Oceanic Technology 20(8): 1685-1696.

Lu, Y., Ueyoshi, K., Kohl, A., Remy, E., Lorbacher, K. and Stammer, D. (2002). Input data sets for the ecco global $1^{o}$ woce synthesis, The ECCO Report Series 18. Available at http://www.ecco-group.org/reports.html.

Macdonald, A. (1998). The global ocean circulation: a hydrographic estimate and regional analysis, Progress in Oceanography 41(3): 281-382.

Marotzke, J., Giering, R., Zhang, K., D, D. S., Hill, C. and Lee, T. (1999). Construction of the adjoint mit ocean general circulation model and application to atlantic heat transport sensitivity, J. Geophys. Res. 104(C12): 29529-29547.

Marsh, R., Nurser, A. J. G., Megann, A. P. and New, A. L. (2000). Water mass transformation in the southern ocean of a global isopycnal coordinate gcm, Journal of Physical Oceanography 30(5): 1013-1045.

Marshall, J., Adcroft, A., Hill, C., Perelman, L. and Heisey, C. (1997). A finitevolume, incompressible Navier-Stokes model for studies of the ocean on parallel computers, J. Geophys. Res. 102(C3): 5753-5766.

Marshall, J., Hill, C., Perelman, L. and Adcroft, A. (1997). Hydrostatic, quasihydrostatic and non-hydrostatic ocean modeling, J. Geophys. Res. 102(C3): 57335752.

Marshall, J., Olbers, D., Ross, H. and Wolfgladrow, D. (1993). Potential vorticity constraints on the dynamics and hydrography of the southern-ocean, J. Phys. Oceanogr. 23(3): 465-487.

Marshall, J. and Radko, T. (2005). A model of the upper branch of the meridional overturning of the southern ocean. To appear in Progress in Oceanography, available at http://paoc.mit.edu/paoc/marshall/latest_papers.htm. 
McIntosh, P. C. and McDougall, T. J. (1996). Isopycnal averaging and the residual mean circulation, Journal of Physical Oceanography 26(8): 1655-1660.

Menemenlis, D., Cheng, B., Fukumori, I., Lee, T., Koehl, A., Stammer, D., Adcroft, A., Heimbach, P., Hill, C., Zhang, J., Henze, C. and Taft, J. (2003). The ECCO high-resolution global-ocean state estimation initiative, Eos Trans. AGU 84(52): OS21H-02. Available at http://ecco.jpl.nasa.gov/cube_sphere/.

NOAA (1988.). Data announcement 88-mgg-02: Digital relief of the surface of the earth. National Geophysical Data Center, Boulder, Colorado.

Olbers, D., Borowski, D., Volker, C. and Wolff, J.-O. (2004). The dynamical balance, transport and circulation of the antarctic circumpolar current, Antarctic Science 16(4): 439-470.

Olbers, D. and Eden, C. (2003). A simplified general circulation model for a baroclinic ocean with topography. part i: Theory, waves, and wind-driven circulations, Journal of Physical Oceanography 33(12): 2719-2737.

Olbers, D. and Visbeck, M. (2005). A model of the zonally averaged stratification and overturning in the southern ocean, Journal of Physical Oceanography 35(7): 11901205.

Orsi, A. H., Whitworth, T. and Nowlin, W. D. (1995). On the meridional extent and fronts of the antarctic circumpolar current, Deep Sea Research 42(5): 641-673.

Patra, P. K., Maksyutov, S., Ishizawa, M., Nakazawa, T., Takahashi, T. and Ukita, J. (2005). Interannual and decadal changes in the sea-air co2 flux from atmospheric co2 inverse modeling, Global Biogeochemical Cycles 19(4): Art. No. GB4013.

Pedlosky, J. (1987). Geophysical Fluid Dynamics, 2 edn, Springer-Verlag, BerlinHeidelberg-New York.

Penduff, T., Barnier, B., Kerbiriou, M.-A. and Verron, J. (2002). How topographic smoothing contributes to differences between the eddy flows simulated by sigma and geopotential-coordinate models., J. Phys. Oceanogr. 32: 122-137. 
Polzin, K. L. and Firing, E. (1997). Estimates of diapycnal mixing using ladep and ctd data from i8s, WOCE Notes 29: 39-42.

Ponte, R. M., Wunsch, C. and Stammer, D. (2006). Spatial mapping of time-variable errors in jason-1 and topex/poseidon surface topography measurements, J. Atmos. Oceanic Tech., submitted. .

Press, W. H., Flannery, B. P., Teukolsky, S. A. and Vetterling, W. T. (1992). Numerical Recipes: The Art of Scientific Computing, 2nd ed., Cambridge University Press, Cambridge, UK.

Reid, J. L. (2003). On the total geostrophic circulation of the indian ocean: Flow patterns, tracers and transports, Progress in Oceanography 56: 137-186.

Reynolds, R. W., Rayner, N. A., Smith, T. M., Stokes, D. C. and Wang, W. (2002). An improved in situ and satellite sst analysis for climate, J. Climate 15: 1609-1625.

Rhein, M., Fischer, J., Smethie, W. M., Smythe-wright, D., Weiss, R. F., Mertens, C., Min, D. H., Fleischmann, U. and Putzka, A. (2002). Labrador sea water: Pathways, cfc inventory, and formation rates, Journal of Physical Oceanography 32(2): 648-665.

Rintoul, S. R., Hughes, C. W. and Olbers, D. (2001). The antarctic circumpolar current system, in G. Siedler, J. Church and J. Gould (eds), Ocean Circulation and Climate, Academic Press, pp. 271-302.

Robbins, P. and Toole, J. (1997). The dissolved silica budget as a constraint on the meridional overturning circulation of the indian ocean, Deep-Sea Research 44: 879906.

Russell, J. L., Stouffer, R. J. and Dixon, K. W. (2005). Intercomparison of the southern ocean circulations in the ipcc coupled model control simulations, Journal of Climate, submitted . 
Saunders, P. and Thompson, S. (1993). Transport, heat and freshwater fluxes within a diagnostic model (fram), J. Phys. Oceanogr. 23: 452-464.

Schmitz, W. J. (1995). On the interbasin-scale thermocline circulation, Reviews of Geophysics 33: 151-173.

Sloyan, B. M. and Rintoul, S. R. (2001a). Circulation, renewal, and modification of antarctic mode and intermediate water, J. Phys. Oceanogr. 31(4): 1005-1030.

Sloyan, B. M. and Rintoul, S. R. (2001b). The southern ocean limb of the global deep overturning circulation, J. Phys. Oceanogr. 31: 143-173.

Smith, W. and Sandwell, D. (1997). Global sea floor topography from satellite altimetry and sparse shipboard bathymetry, Science 277: 1956-1961.

Stammer, D., Davis, R., Fu, L.-L., Fukumori, I., Giering, R., Lee, T., Marotzke, J., Marshall, J., Menemenlis, D., Niiler, P., Wunsch, C. and Zlotnicki, V. (1999). The consortium for estimating the circulation and climate of the ocean (ecco): Science goals and task plan, The ECCO Report Series 1, Scripps Instituttion of Oceanography, 9500 Gilman Dr., La Jolla, CA 92093-0239; also available at http://www.ecco.ucsd.edu/report/report_1/report_1.html.

Stammer, D., Wunsch, C., Giering, R., Eckert, C., Heimbach, P., Marotzke, J., Adcroft, A., Hill, C. N. and Marshall, J. (2002). Global ocean circulation during 1992-1997, estimated from ocean observations and a general circulation model, $J$. Geophys. Res. 107(C9): 3118.

Stammer, D., Wunsch, C., Giering, R., Eckert, C., Heimbach, P., Marotzke, J., Adcroft, A., Hill, C. N. and Marshall, J. (2003). Volume, heat, and freshwater transports of the global ocean circulation 1993-2000, estimated from a general circulation model constrained by World Ocean Circulation Experiment (WOCE) data, J. Geophys. Res. 108(C1): 3007-3029.

Stevens, I. and Stevens, D. P. (1999). Passive tracers in a general circulation model of the southern ocean, Annales Geophysicae 17: 971-982. 
Talley, L. D., Reid, J. L. and Robbins, P. E. (2003). Data-based meridional overturning streamfunctions for the global ocean, Journal of Climate 16(19): 3213-3226.

Tansley, C. E. and Marshall, D. P. (2001). Flow past a cylinder on a beta-plane, with application to gulf stream separation and the antarctic circumpolar current, J. Phys. Oceanogr. 31: 3274-3283.

The FRAM Group (1991). An eddy-resolving model of the southern ocean, EOS, Transactions of the American Geophysical Union 72(15): 169-174.

Tomczak, M. and Godfrey, J. S. (1994). Regional Oceanography: An Introduction, Pergamon, New York.

Tomczak, M. and Liefrink, S. (2005). Interannual variations of water mass volumes in the southern ocean, Journal of Atmospheric and Ocean Science 10(1): 31-42.

Toole, J. and Warren, B. A. (1993). A hydrographic section across the subtropical south indian ocean., Deep-Sea Research 40(10): 1973-2019.

Visbeck, M., Marshall, J., Haine, T. and Spall, M. (1997). Specification of eddy transfer coefficients in coarse-resolution ocean circulation models, J. Phys. Oceanogr. 27(3): 381-402.

Warren, B. A., LaCasce, J. H. and Robbins, P. E. (1998). Comments on "on the obscurantist physics of "form drag" in theorizing about the circumpolar current"reply, Journal of Physical Oceanography 28(8): 1655-1658.

Webb, D. J. and de Cuevas, B. A. (2006). On the fast response of the southern ocean to changes in the zonal wind, Ocean Science Discussions 3: 471-501.

Wunsch, C. (1996). The Ocean Circulation Inverse Problem, Cambridge University Press, New York.

Wunsch, C. (1998). The work done by the wind on the oceanic general circulation, J. Phys. Oceanogr. 28(11): 2332-2340. 
Wunsch, C. and Ferrari, R. (2004). Vertical mixing, energy, and the general circulation of the ocean, Annual Review of Fluid Mechanics 36: 281-314.

Wunsch, C. and Heimbach, P. (2005a). Decadal changes in the north atlantic meriodional overturning and heat flux. Submitted. See http://ocean.mit.edu/ ${ }^{\sim}$ cwuncsh.

Wunsch, C. and Heimbach, P. (2005b). Practical global oceanic state estimation. Submitted for publication. See http://ocean.mit.edu/ ${ }^{\sim}$ cwuncsh. 